\section{FIV as a Model for HIV: An Overview}

\author{
ELLEN E. SPARGER
}

\section{INTRODUCTION}

Animal models for human immunodeficiency virus (HIV) infection play a key role in understanding the pathogenesis of AIDS and the development of therapeutic agents and vaccines. As the only lentivirus that causes an immunodeficiency resembling that of HIV infection, in its natural host, feline immunodeficiency virus (FIV) has been a unique and powerful model for AIDS research. FIV was first described in 1987 by Niels Pedersen and co-workers as the causative agent for a fatal immunodeficiency syndrome observed in cats housed in a cattery in Petaluma, California. ${ }^{1,2}$ Since this landmark observation, multiple studies have shown that natural and experimental infection of cats with biological isolates of FIV produces an AIDS syndrome very similar in pathogenesis to that observed for human AIDS. FIV infection induces an acute viremia associated with Tcell alterations including depressed CD4:CD8 T-cell ratios and CD4 T-cell depletion, peripheral lymphadenopathy, and neutropenia. ${ }^{3-13}$ In later stages of FIV infection, the host suffers from chronic persistent infections that are typically self-limiting in an immunocompetent host, as well as opportunistic infections, chronic diarrhea and wasting, blood dyscracias, significant CD4 T-cell depletion, neurologic disorders, and B-cell lymphomas. ${ }^{2,6,9,12-14}$ Importantly, chronic FIV infection induces a progressive lymphoid and CD4 T-cell depletion in the infected cat. The primary mode of natural FIV transmission appears to be blood-borne facilitated by fighting and biting. ${ }^{13,15}$ However, experimental infection through transmucosal routes (rectal and vaginal mucosa and perinatal) have been well documented for specific FIV isolates. ${ }^{16-23}$ Accordingly, FIV disease pathogenesis exhibits striking similarities to that described for HIV-1 infection. ${ }^{24}$ Recent observations regarding functions of FIV accessory and structural genes, FIV

ELLEN E. SPARGER - School of Veterinary Medicine, University of California Davis, CA 95616 
tropism, and immunopathogenesis have further corroborated similarities shared by FIV and HIV-1. This chapter will serve as an overview of the FIV animal model for HIV AIDS and as such, will focus on FIV molecular biology and virology and address recent developments in FIV viral vector development as well as nondomestic FIV biology. FIV pathogenesis, vaccine development, and antiviral therapies are critical topics for discussion regarding the value of the FIV animal model and will also be described briefly in this overview, but will be examined in more significant detail in subsequent chapters.

\section{FIV GENOME, STRUCTURE, AND GENE FUNCTION}

\section{Virion Structure}

The morphology of the FIV virus particle is similar to that of other lentiviruses. ${ }^{1,14,25}$ The mature extracellular virion is spherical to ellipsoid, 100 to $125 \mathrm{~nm}$ in diameter, and bordered by an outer envelope with poorly defined short projections or knobs. An elongated conical shell surrounds an eccentrically positioned electron-dense viral nucleoid. A polygonal electron-lucent halo is often visible between the core and a granular layer located just inside the envelope. Similar to other lentiviruses, the density of FIV was shown to be $1.15-1.17 \mathrm{~g} / \mathrm{cm}^{3}$ by continuous sucrose gradient centrifugation. ${ }^{26}$

Typical of other retroviruses, the FIV genome consists of an identical pair of single-stranded RNA molecules that are approximately 9,200 bases in length and densely packed within the virion by their association with nucleocapsid protein (NC, p7) ${ }^{27,28} \mathrm{~A} t-\mathrm{RNA}^{\text {lys }}$ is hydrogen-bonded to each RNA molecule at the primer binding site (PBS) located within the $5^{\prime}$ terminal 180 bases of the genome and serves as the primer for negative strand reverse transcription. The ribonucleoprotein complex is contained within a protein core largely composed of the viral capsid protein (CA, p24) that is associated with and surrounded by a roughly spherical shell consisting of myristylated matrix protein (MA, p14). Also contained in the viral capsid are viral enzymes involved in particle maturation and replication of the viral RNA genome including protease $(\mathrm{PR})$, reverse transcriptase $(\mathrm{RT})$, integrase (IN), and dUTPase (DU). ${ }^{29-31} \mathrm{NC}, \mathrm{CA}$, and MA are expressed from the gag gene, while PR, RT, DU, and IN are products of the pol gene. Outside the matrix coat is a lipid bilayer of the virion envelope, which confers the characteristic icosahedral morphology to the enveloped retrovirus. Embedded within the lipid bilayer are viral envelope glycoproteins, with the transmembrane subunit (TM, gp40) present as a single-pass transmembrane protein anchor, and the surface unit (SU, gp95) as an entirely extravirion protein bound to $\mathrm{TM} .{ }^{27}$ Both $\mathrm{TM}$ and $\mathrm{SU}$ are the products of 
the $e n v$ gene. Env gene products mediate binding of the virus to cell surface receptors and fusion with the target cell membrane $e^{32-36}$ and are critical targets for host humoral and cellular immune responses.

\section{Genome Organization and Expression}

Sequence organization of the FIV genome is similar to that of HIV-1 and other lentiviruses. ${ }^{37,38}$ Flanked by two long terminal repeats (LTR) at both ends, the FIV proviral DNA genome contains three large open reading frames (ORFs), gag, pol, and env, encoding internal structural proteins, RT and other viral enzymes, and envelope proteins, respectively, as well as various small ORFs encoding regulatory and accessory proteins (Figure 1). The FIV genome also contains nonencoding regulatory sequences important for virus replication. These sequences include transcriptional elements within the LTRs, a posttranscriptional regulatory sequence located in $3^{\prime}$ half of the genome, ${ }^{39-43}$ and encapsidation determinants within the U5 domain and the first 90-300 nucleotides of gag. $^{44,45}$ Other critical noncoding sequences include the central polypurine tract (cPPT) involved in priming plus-strand DNA synthesis and the central termination sequence (CTS) important for formation of a central DNA flap during reverse transcription. ${ }^{46}$

The approximately 355 bp long FIV LTR accommodates multiple regulatory sequences and is composed of three domains designated U3, R, and U5. Located at each terminus of the proviral DNA genome, the LTRs are bordered by two bp-inverted repeats. Critical transcriptional regulatory sequences located in the FIV U3 domain consist of enhancer elements including AP-1, ATF (also known as the cAMP response element or CRE), and cEBP sites. These cis-acting elements have been shown to be important for FIV LTR promoter activity in vitro and for virus replication in vitro

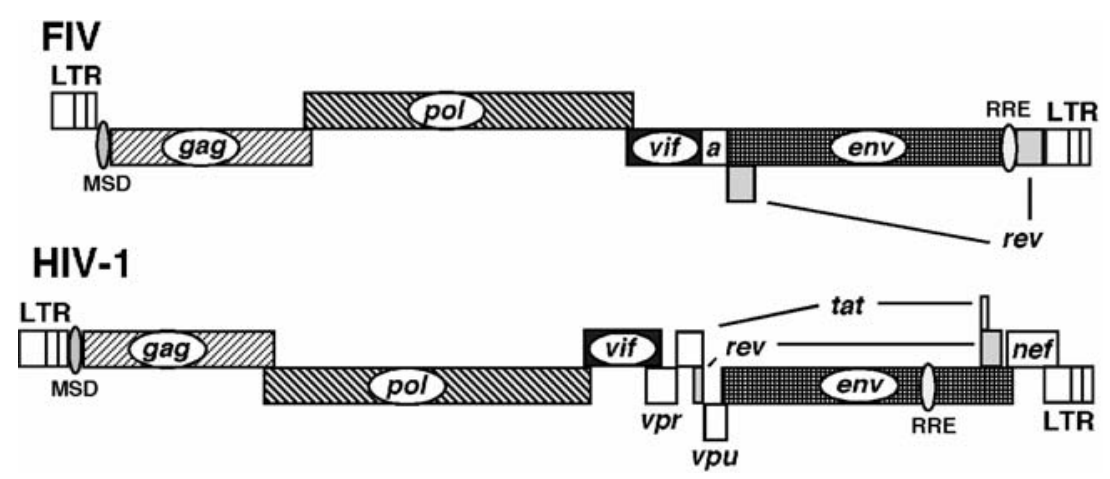

FIGURE 1. Comparison of FIV and HIV-1 genomic organization. 
and in vivo..$^{39,40,43,47-49}$ Previous studies also demonstrated binding of these specific LTR sequences by cellular proteins using DNase I footprinting and gel shift assays. ${ }^{39,50}$ Although cis-acting transcriptional elements within the FIV U3 domain differ from those described as critical for the HIV LTR (NFא $\beta$ and SP1) ${ }^{51,52}$ FIV-encoded AP-1 and ATF sequences are positive regulatory elements that respond to host cell activation states ${ }^{40}$ a property also shared by the NFא $\beta$ site within the HIV-1 LTR. Other retroviruses encoding LTRs regulated by ATF/CREB family of transcription factors include human T-lymphotropic virus type (HTLV-1) and bovine leukemia virus (BLV) ${ }^{53-56}$ Similarly, an AP-1 site encoded by the visna virus LTR was found to be critical for basal activity and for transactivation of the viral LTR resulting from interactions of the visna virus putative transcriptional transactivator (Tat) protein with cellular transcription factors Fos and Jun. ${ }^{57-59}$ Interestingly the structure of Orf-A, an FIV accessory gene product previously regarded to be a Tat protein, is very similar to that of visna virus Tat. ${ }^{60}$ Collectively, these observations suggest that FIV LTR promoter activity may be regulated by multiple cell activation pathways involving possible interactions between a viral accessory protein (Orf-A) with cellular proteins that bind either AP-1, ATF, or cEBP elements. However, a definitive characterization of these potential interactions has not yet been reported. Knowledge of potential complex interactions between the FIV LTR and viral and cellular proteins that are most likely involved in regulation of FIV expression is still rudimentary at best.

The FIV LTR is also distinguished from the HIV-1 LTR by its strong basal promoter activity that does not require activation by a viral transactivator. ${ }^{39,40,43,61}$ Similarly, other animal lentiviruses including caprine arthritis encephalitis virus (CAEV) and visna virus (VV) also encode LTR promoters capable of high basal levels of transcription in the absence of a viral transactivator. ${ }^{62,63}$ In contrast, LTRs encoded by primate lentiviruses including HIV and simian immunodeficiency virus (SIV), include a transcriptional element designated TAR (Tat responsive element) that possesses a stem-loop structure for binding of the virus encoded transactivator Tat and other cellular proteins. ${ }^{64,65}$ Tat transactivation of the HIV LTR is required for elongation of initiated RNA transcripts and for efficient transcription of viral genes. The FIV LTR does not contain a stem-loop structure of similar complexity nor does FIV encode a transcriptional transactivator with structure or activity similar to that described for HIV Tat. ${ }^{39,40,43,61}$ Previous reports have proposed that the gene product of FIV accessory gene orf-A may encode a viral transactivator or FIV Tat. However, these studies have generated conflicting data regarding the ability of FIV-encoded OrfA to transactivate the FIV LTR and revealed either no effect, a small effect, or a moderate effect imposed by Orf-A on FIV LTR-directed gene expression in transient expression assays. ${ }^{39,40,43,61,66,67}$ Taken together, these data suggest that the FIV LTR may be regulated in part by accessory protein Orf- 
A although by mechanisms unlike those described for the HIV LTR and HIV Tat.

Examination of FIV mRNA species from infected cells revealed the presence of at least five short multiply spliced transcripts in addition to unspliced genomic RNA and env-containing singly spliced transcripts. ${ }^{42,66,68}$ Nuclear export of unspliced and singly spliced FIV mRNA transcripts involves binding of a posttranscriptional regulatory sequence, designated the rev response element (RRE), by the FIV regulatory protein Rev. The FIV RRE is a 243 nucleotide sequence that forms a stem-loop structure within viral mRNA species and serves as a binding site for FIV regulatory protein Rev and is structurally and functionally similar to the HIV-1 RRE. ${ }^{41}$ However, the FIV RRE is located at the $3^{\prime}$ terminus of the env gene and partially overlaps the 3' LTR, whereas the HIV-1 RRE is positioned between the junction of the SU and TM open reading frames within env. Binding of the FIV RRE by the viral protein Rev is critical for cytoplasmic accumulation of unspliced and singly spliced FIV mRNA transcripts and for FIV structural (Gag and Env) and enzymatic ( $\mathrm{Pol}$ ) protein expression. ${ }^{41,69}$

\section{FIV Structural and Enzymatic Proteins}

FIV Gag proteins are necessary and sufficient for the formation of the noninfectious virus-like particles. Similar to other lentivirus systems, expression of FIV Gag polyprotein precursor (p50) from the gag gene within the unspliced genome RNA is dependent on viral Rev. Nevertheless, the FIV Gag polyprotein, when expressed in the absence of other viral structural proteins such as $e n v$ gene products, is capable of self-assembly into virus particles that are released from the plasma membrane of Gag-expressing cells. ${ }^{70}$ Formation of mature virus particles, however, requires cleavage of the FIV Gag polyprotein by virus-encoded PR during or shortly after budding from the cell to generate three mature Gag proteins: MA, CA, and NC (Figure 2) ${ }^{28,71}$ Examination of the proteolytic sites within the FIV Gag polyprotein revealed processing of Gag proteins similar to that for HIV-1 Gag proteins, and in particular to HIV-1 CA protein. ${ }^{71}$ Within the mature virion, $\mathrm{MA}$ is attached to the viral lipid membrane while $\mathrm{CA}$ forms the virus core, and $\mathrm{NC}$ is present in the virus core in a ribonucleoprotein complex with the viral RNA genome. ${ }^{72}$

Reports describing studies focused on FIV Gag protein processing and characterization of functional domains are still limited. ${ }^{27,28,70,71}$ Similar to HIV MA, myristylation of FIV MA is required for targeting of MA to the plasma membrane during late events of viral assembly. ${ }^{70,71}$ In contrast to observations for HIV-1 MA, targeting of FIV MA to the cellular plasma membrane is not dependent on a N-terminal polybasic domain that is present in both FIV and primate lentivirus MA proteins. Instead, this conserved polybasic domain (lysine-rich) in FIV MA appears necessary for 


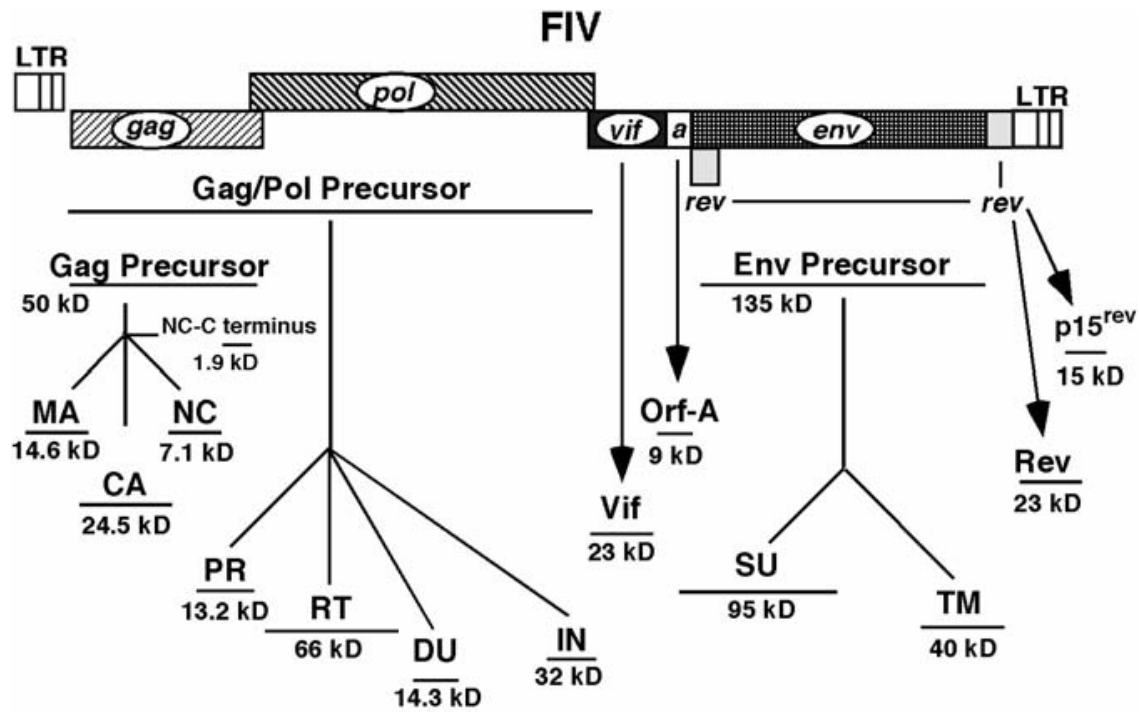

FIGURE 2. Proteins encoded by FIV. Sizes of primary gene products after processing are shown.

either particle assembly or release. ${ }^{70}$ The role of FIV MA in other steps of virus replication, where HIV-1 MA is thought to be important, such as early postentry events, ${ }^{73}$ has not yet been determined.

The principal function of FIV NC involves encapsidation of full-length, unspliced viral genomic RNA into virions. Similar to other retroviruses, FIV NC protein contains two copies of a zinc finger motif, which has been characterized as a zinc-binding moiety for HIV-1 RNA. ${ }^{74}$ Although studies describing FIV NC interactions with viral RNA are few, recent observations suggest that FIV NC binds to viral RNA at, or upstream of, the PBS and may thereby initiate RNA dimerization and promote initiation of minus strand DNA. ${ }^{75}$ However, the role of NC in viral RNA encapsidation or determinants responsible for FIV NC binding of RNA have not yet been reported. Interestingly, examination of proteolytic cleavage of $\mathrm{NC}$ revealed a secondary cleavage site within the C-terminus of $\mathrm{NC}$ that produces a mature NC $7.1 \mathrm{kD}$ protein and a C-terminus $1.9 \mathrm{kD}$ protein. ${ }^{71}$ Although further characterization of this C-terminus $1.9 \mathrm{kD}$ protein has not yet been described, amino acid sequence of this cleavage product contains a PSAP motif similar to the PTAP motif also characterized as a "late" or L domain encoded by the HIV-1 p6 Gag protein. ${ }^{76}$ This motif within HIV-1 p6 functions in virus particle budding and release $\mathrm{e}^{77,78}$ and is a binding motif for proteins encoding WW domains. ${ }^{79}$ Interactions of the L domain of HIV-1 p6 with cellular protein TSG101 are reported to be critical for HIV-1 
budding ${ }^{80-82}$ and are under investigation as potential targets for antiviral therapeutics. Examination of the significance and potential function of this C-terminal cleavage product of FIV NC as a L domain for FIV Gag may be warranted and provide another lentiviral model for characterizing cellular proteins important for lentivirus assembly and release.

In addition to MA, CA, and NC proteins encoded by FIV gag, the FIV Gag polyprotein contains a CA-NC spacer region shown to regulate temporal Gag processing for several retroviruses including HIV-1, bovine immunodeficiency virus (BIV), and Rous sarcoma virus (RSV) ${ }^{83}$ The FIV CA-NC spacer region consists of nine residues and contains a LAEAL motif also found in the HIV-1 CA-NC spacer region and reported to be indispensable for HIV-1 Gag assembly. ${ }^{84,85}$ Although a recent study showed that the FIV CA-NC spacer region was capable of functionally replacing the BIV CA-NC spacer region for BIV Gag assembly, studies examining the FIV CANC spacer region in FIV Gag assembly have not been reported. This observation, however, suggests that FIV Gag may provide another model for examination of this Gag motif in HIV-1 assembly.

The FIV pol gene positioned downstream of gag, encodes four enzymes: protease (PR), reverse transcriptase (RT), dUTPase (DU), and integrase (IN) (Figure 2). FIV pol overlaps the gag gene by 109 nucleotides and is in a -1 reading frame with respect to that of gag. Similar to other retroviruses, pol is translated as a Gag-Pol fusion polyprotein produced by ribosomal frameshifting ${ }^{86}$ facilitated by a consensus frameshift signal sequence of GGGAAAC within the gag-pol overlap region, together with a sequence displaying potential for a pseudoknot tertiary structure immediately downstream of the signal sequence.$^{87}$ The Pol polypeptide of the GagPol fusion precursor protein is cleaved by viral PR into functional enzymes during virus assembly.

FIV PR is a $14.3 \mathrm{kD}$ protein that facilitates processing of Gag and GagPol polyproteins into individual structural and enzymatic proteins during assembly and maturation of the virus particle ${ }^{88}$ Focus on antiviral therapies targeted to HIV-1 PR have promoted interest in FIV PR as a model for design of protease inhibitors as well as structural studies characterizing FIV PR. Based on three-dimensional crystal structure analysis, FIV PR is a homodimeric aspartyl proteinase with quarternary structures very similar to those of HIV-1 PR despite a conservation of only 27 amino acids between the two enzymes. ${ }^{89,90}$ However, each monomer of FIV protease is composed of 116 amino acids compared to 99 amino acids for HIV-1 PR. Regardless of similarities observed between FIV PR and HIV-1 PR, FIV PR exhibits a substrate specificity that is restricted to FIV Gag cleavage sites and excludes sites within HIV-1 Gag. ${ }^{90,91}$ Multiple residue substitutions are required within FIV PR to modify this specificity to include HIV-1 Gag cleavage sites. ${ }^{92-94}$ Furthermore, residues peripheral to the active site of PR, as well as those within the active site, influence binding of substrate by stabilizing 
crucial residues within the active site that directly contact substrate and may account for differences in substrate specificities observed between FIV and HIV-1 PR activities. ${ }^{95}$ Regardless of disparate substrate specificity displayed by FIV PR and HIV-1 PR, similarities in their structure have been utilized in the development of broad-based inhibitors that will bind both HIV PR and FIV PR. ${ }^{94,96,97}$ These comparative analyses should significantly increase the understanding of the molecular basis for lentivirus PR substrate specificity and may possibly facilitate the development of PR inhibitors less susceptible to resistance development.

Reverse transcriptases are encoded by all retroviruses and are RNAdependent DNA polymerases that reverse transcribe viral genomic RNA into a double-stranded proviral DNA copy that is subsequently integrated into the host cellular genomic DNA. ${ }^{98}$ FIV RT is comparable to HIV-1 RT in amino acid sequence, structure and physical properties, catalytic activities, and susceptibility to multiple nucleoside analogs. ${ }^{99-101}$ Amino acid sequence analysis reveals a $48 \%$ identity and $67 \%$ similarity between HIV1 RT and FIV RT. ${ }^{101}$ Like HIV-1 RT, FIV RT exists as a heterodimer consisting of a $66 \mathrm{kD}$ subunit (p66) and a $51 \mathrm{kD}$ subunit (p51), each of which contains a common $\mathrm{N}$-terminus and are present in equimolar concentrations. ${ }^{100}$ The p51 subunit is generated by cleavage of the RNase $\mathrm{H}$ domain from C-terminus of p66. The RNase $\mathrm{H}$ domains in FIV RT and HIV-1 RT function in reverse transcription to degrade RNA from the DNA-RNA hybrid. ${ }^{102}$ Importantly, FIV RT and HIV-1 RT exhibit a similar susceptibility to multiple nucleoside analogs. ${ }^{100,103-105}$ However, FIV RT resistance to specific nucleoside analogs such as $3^{\prime}$-azido- $3^{\prime}$-deoxythymidine (AZT), 2', $3^{\prime}$ dideoxyinosine (ddI), 2', $3^{\prime}$-didehydro- $3^{\prime}$-deoxythymidine (d4T), and 2', $3^{\prime}$ dideoxycytidine (ddC) does not map to homologous residues within similarly drug-resistant HIV-1 RT mutants. In contrast, FIV RT and HIV-1 RT susceptibilities to nucleoside analog (-)-+-L-2', $3^{\prime}$-dideoxy-3'-thiacytidine (3TC) map to corresponding codon, M184 in the YMDD active site of the RT palm subdomain. ${ }^{106,107}$ Other studies also revealed that a unique 3TCresistant FIV mutant encoded a novel proline to serine change at position 156, analogous to proline residue 157 residing within the template grip of HIV-1 RT. ${ }^{108}$ This observation suggested that mutations within a region (template grip) close to, but distinct from the RT active site could influence substrate recognition, a conclusion further substantiated by examination of homologous HIV-1 RT mutant P157S. ${ }^{103}$ Furthermore, functional studies assessing chimeric RT molecules composed of FIV and HIV-1 p51 and p66 subunits demonstrated the importance of p51 in maintaining optimal structural integrity of RT and shed some light on significance of the p51 subunit. ${ }^{109,110}$ These studies also produced observations showing the lack of FIV RT sensitivity to non-nucleoside RT inhibitors (NNRTI) found to block HIV-1 replication, although amino acids lining the NNRTIspecific pocket of HIV-1 RT exhibit a higher similarity to the correspond- 
ing FIV RT residues than to HIV-2 RT. ${ }^{109,110}$ Catalytic activity of HIV/FIV chimeric RTs was also found to be significantly decreased compared to wild type HIV and FIV RTs, despite similarities observed between the two molecules. ${ }^{109}$ In summary, similarities and differences observed between HIV1 and FIV RT have generated somewhat limited support for use of FIV RT as a model for HIV-1 RT-targeted drug design and studies of drug resistance both in cell culture and in vivo. ${ }^{111}$

A DU gene product is expressed from the pol gene in genomes from nonprimate lentiviruses including FIV and the type-D retroviruses, but not from primate lentiviruses. ${ }^{30}$ FIV DU resides immediately downstream of RT in the Pol polyprotein and is packaged in active form in FIV virions. DU catalyzes the hydrolysis of dUTP to dUMP and inorganic pyrophosphate (PPi), and is believed to minimize misincorporation of dUTP into DNA, which can be mutagenic. ${ }^{112}$ For those viruses encoding a DU, enzymatic activity is required for productive viral replication in cells such as primary macrophages that express low dUTPase activity. ${ }^{113-115}$ Infection of cats with a DU mutant of FIV resulted in fivefold increase in the number of mutations observed in the viral genome. ${ }^{14,115}$ Although HIV-1 does not encode a dUTPase activity, recruitment of a cellular DNA repair enzyme, uracil DNA glycosylase (UNG), into HIV-1 virions by accessory protein Vpr, also acts to modulate viral mutation rate. ${ }^{116-119}$ Accordingly, HIV Vpr activity imparts a similar effect to that of FIV DU on virus replication but through a different mechanism.

Integration of double stranded proviral DNA into the host genome is a function of all retroviral integrase proteins and is a distinguishing feature of retrovirus replication. ${ }^{120}$ FIV IN is a $32 \mathrm{kD}$ protein that is approximately $37 \%$ identical to HIV-1 IN by amino acid sequence. ${ }^{121}$ Similar to other retroviral IN proteins, FIV IN contains three domains including an N-terminal domain, a central catalytic core domain, and a C-terminal domain. ${ }^{122}$ Studies testing activity of a recombinant FIV IN expressed in E. coli revealed that FIV IN exhibits a relaxed sequence requirement for site-specific cleavage and integration of viral DNA termini and is active on FIV, HIV, and Moloney murine leukemia virus (MoMLV) DNA termini. ${ }^{121}$ A difference noted between FIV IN and HIV-1 IN was their choice of nucleophiles in vitro with FIV IN preferentially using the 3' OH viral DNA ends and HIV1 IN using $\mathrm{H}_{2} \mathrm{O}$ and glycerol. In vitro analyses of recombinant FIV IN also demonstrated that the central catalytic core domain determined target site selection and the importance of a central aspartic acid (D118) in 3' terminus processing and joining activities. ${ }^{123,124}$ Virus replication studies testing FIV IN mutants in the context of FIV vectors showed that mutation of either D66 and or both D66 and D118 within the catalytic core domain blocked transduction of dividing fibroblast and integrations, as would be predicted for type I IN mutants. ${ }^{125}$ These observations for mutants involving FIV IN residues D66 and D118 that correspond to D64 and D116 in 
the catalytic triad of HIV-1 IN, characterized properties of catalytic core IN mutants for a non-HIV-1 lentivirus, and verified similarities between FIV and HIV-1 IN.

In addition to a direct role in integration of proviral DNA into host cell genomic DNA, HIV-1 IN is a component of the PIC that also contains newly synthesized proviral DNA, viral MA and Vpr, and the viral central DNA flap. Each of these viral components are thought to contribute to nuclear import of the $\mathrm{PIC}^{126}$ and IN may play the primary role. ${ }^{127}$ Similar to HIV-1 IN, FIV IN exhibits karyophilic properties. Determinants encoded by FIV IN for nuclear import map to a N-terminal zinc-binding domain and to a region rich with basic residues near the $\mathrm{C}$-terminal domain, rather than to a canonical nuclear localization signal (NLS) ${ }^{128}$ The NLS for HIV-1 IN is also thought to involve a bipartite signal that instead includes a 13 residue peptide within the central core domain of IN, but does not include the Nterminal zinc-binding domain, suggesting that mechanisms for nuclear import of HIV-1 and FIV integrase molecules are different. ${ }^{128}$

The $e n v$ gene of FIV and other lentiviruses is the most diverse viral gene in size and sequence. ${ }^{73}$ Lentivirus envelope proteins play a major role in the virus life cycle by encoding determinants that interact with cell surface receptor and mediate fusion between the lipid bilayer of the viral envelope and host cell plasma membrane. Accordingly, variation in viral envelope proteins, particularly the surface glycoprotein, affects virus host cell tropism and fusogenicity, as well as virus replication. In addition, the Env glycoproteins contain epitopes that elicit immune responses important for both diagnosis and protective immunity.

FIV env expression from a singly spliced mRNA is Rev-dependent, similar to other structural proteins. In contrast to primate lentivirus Env proteins, FIV Env and other nonprimate lentiviruses Env proteins encode a lengthy $\mathrm{N}$-terminal presequence upstream of the hydrophobic region of the Env signal peptide (Figure 3). ${ }^{37,38,129,130}$ This N-terminal presequence of FIV Env, containing 149 amino acids, represents a $20 \mathrm{kD}$ polypeptide and includes the $\mathrm{L}$ region of $e n v$ that encodes the N-terminal exon of FIV Rev. ${ }^{41,69}$ The early gene product of FIV env is a full-length uncleaved precursor $145-150 \mathrm{kD}$ glycoprotein that is subsequently processed to a $130 \mathrm{kD}$ precursor (gp130) by cleavage of the N-terminal $20 \mathrm{kD}$ polypeptide and hydrophobic signal sequence. ${ }^{129,130}$ Precursor gp130 is then transported to the Golgi and proteolytically cleaved to produce mature FIV surface glycoprotein (SU) gp95 and characteristic hydrophobic membrane-spanning glycoprotein (TM) gp40. SU forms a noncovalent association with TM, which anchors the envelope complex to the lipid bilayer. Studies using glycosylation inhibitors have confirmed extensive glycosylation of FIV envelope proteins similar to that observed for HIV Env and demonstrated cell type-specific glycosylation of Env. ${ }^{129,131}$ These studies also verified the role of envelope protein glycosylation in virus infectivity. ${ }^{129}$ Sig- 


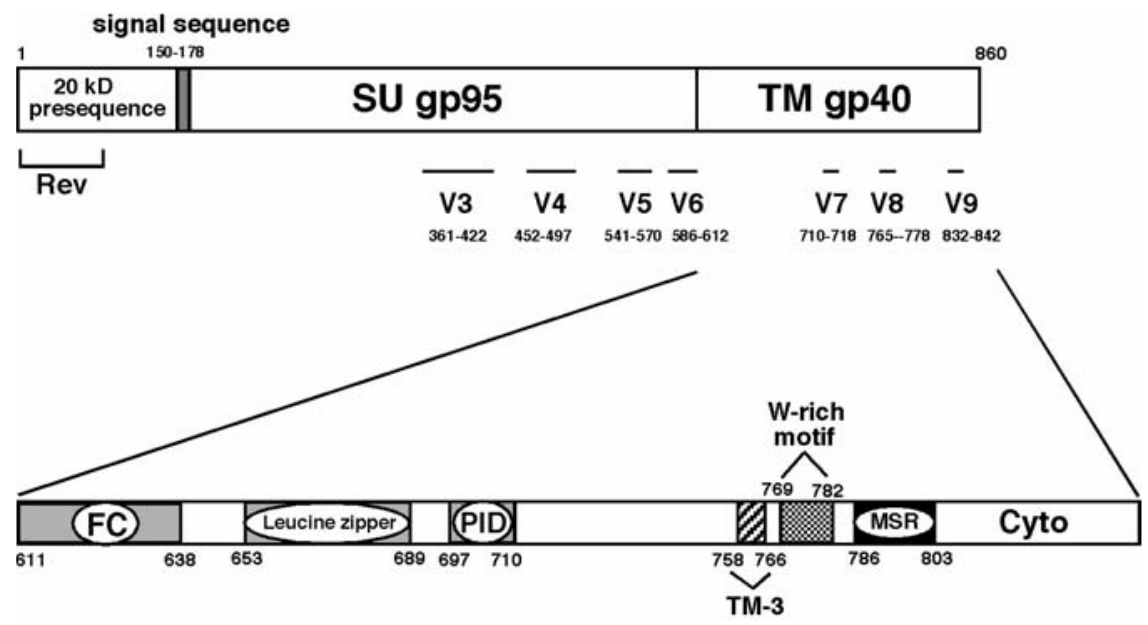

FIGURE 3. Schematic representation of variable regions, functional domains, and various epitopes encoded by FIV env gene products SU and TM. Amino acid positions indicated for each domain or epitope are based on deduced amino acid sequence of FIV molecular clone 34TF10. ${ }^{38}$ Variable regions (V3 through V9) are shown as previously described. ${ }^{134,142}$ Functional domains and epitopes within TM as previously reported, ${ }^{133,137,138,454}$ include: FC, fusion peptide; leucine zipper region; principal immunodominant domain, PID; a TM3 epitope analogous to neutralizing HIV-1 2F5 epitope; a tryptophan (W)-rich motif important for infectivity; the membrane-spanning region, MSR, and the cytoplasmic domain, Cyto.

nificance, structure, or function of this $20 \mathrm{kD} \mathrm{N}$-terminal polypeptide cleavage product, apart from proper Env processing, is not well understood, although one study revealed that partial deletion of this peptide produced a virus unable to infect primary feline astrocytes while still infectious for feline lymphocytes and macrophages. ${ }^{132}$

Structural models based on x-ray crystallography or NMR spectroscopy indicate that HIV-1 envelope proteins form trimers on the viral surface. Furthermore, SU binding of the primary receptor, CD4, results in conformational changes facilitating formation of a ternary complex composed of CD4, SU, and coreceptor molecules ( $\beta$-chemokine receptors CCR-5 and CXCR-4). This ternary complex triggers additional conformation changes in TM that mediate fusion of envelope proteins with the cellular plasma membrane. ${ }^{73}$ Models for spatial folding of FIV Env SU and TM proteins based on predictive algorithms using Env amino acid sequence from multiple isolates, reveal structural similarities to HIV-1 envelope proteins, including conserved and hypervariable domains for both FIV SU and TM. ${ }^{133-135}$ A principal immunodominant domain (PID) within the extracellular region of TM that is conserved among lentivirus transmembrane glycoproteins, including HIV-1 TM, has also been described for FIV TM (Figure 3). ${ }^{133,136}$ Despite conservation of the PID, mutation of this TM 
domain was not found to alter FIV infectivity. ${ }^{136}$ In contrast, disruption of a tryptophan-rich domain also conserved among lentivirus TM proteins and located in the extracellular region immediately upstream of the membrane-spanning domain abrogated virus entry. ${ }^{34}$ Findings from a second study demonstrated a role for the FIV TM tryptophan-rich domain in Envmediated fusion between viral and cellular membranes, thus providing a probable mechanism by which this domain affects virus infectivity. ${ }^{137}$ Finally, synthetic peptides modeled on this tryptophan-rich domain were found to inhibit FIV replication. ${ }^{138}$ These findings parallel other data demonstrating the importance of a similar tryptophan-rich domain encoded by HIV TM for HIV-1 entry and HIV-1 fusiogenic effects ${ }^{139,140}$ and suggest that FIV and HIV-1 TM may share one mechanism necessary for fusion of viral and cellular membranes. However, a more detailed structural analysis of FIV envelope proteins and their interactions with cell surface receptors based on x-ray crystallography will be needed for a thorough comparison of FIV and HIV-1 envelope proteins.

As stated earlier in this chapter, envelope proteins exhibit the greatest diversity in amino acids among all proteins encoded by lentiviruses. By comparison of different HIV isolates, five interspersed conserved (C1 to $\mathrm{C5}$ ) and five variable domains (V1-V5) were identified for HIV-1 env gene. ${ }^{73}$ Similarly, analysis of amino acid sequences derived from the $e n v$ gene of different isolates of FIV resulted in identification of nine variable regions (V1-V9), separated by regions that are more conserved (Figure 3). ${ }^{134,141-143}$ Furthermore, examination of V3, V4, and V5 domains within SU-coding sequence revealed up to $26 \%$ genetic diversity for different isolates and allowed separation of FIV isolates into five clades, or subtypes, designated A to $\mathrm{E}^{134,142,144-150}$ and possibly a sixth subtype arising from a subtype $\mathrm{B}$ cluster found in Texas. ${ }^{151}$ Diversity between subtypes varies from 17 to $26 \%$ whereas variation within a subtype ranges from 2.5 to $15 \%$, findings that are similar to those for HIV-1 isolates. ${ }^{142}$ Although geographic separation is most likely a major factor for emerging genetic diversity of FIV, individual subtypes have been identified in geographically isolated regions of the world (Table I). FIV subtype A and B isolates have been detected in United States, Europe, Japan, and Australia, whereas subtype C variants have been found in North America, Europe, and Taiwan. Subtype A isolates have also been detected in Central and South America ${ }^{149}$ and all three FIV subtypes $\mathrm{A}, \mathrm{B}$, and $\mathrm{C}$ have been isolated from a single location (Munich, Germany). ${ }^{150}$ Subtype D and E isolates, characterized so far, are few and restricted to $\operatorname{Japan}^{147}$ and Argentina ${ }^{145}$ respectively. Multiple genetic analyses indicate that the vast majority of FIV isolates are either subtype A or B. Interestingly, findings from one report revealed twice as many synonymous site changes within FIV subtype B envelope V3 to V4 domains compared to those detected for subtype A envelope, although both subtype A and B envelopes encoded similar numbers of nonsynonymous site changes. ${ }^{150}$ 
TABLE I

Geographic Distribution of FIV Subtypes

\begin{tabular}{ll}
\hline Subtype & \multicolumn{1}{c}{ Location } \\
\hline A & United States, Europe, Japan, Australia, Central and South America \\
B & United States, Europe, Japan, and Australia \\
C & North America, Europe, and Taiwan \\
D & Japan \\
E & Argentina \\
\hline
\end{tabular}

The greater diversity observed for subtype B suggests that this FIV subtype may represent an older virus. Significant diversity of FIV subtype B variants was also shown for viruses isolated from cats in Italy where this subtype is highly prevalent. ${ }^{144}$ Findings from this study were generated by phylogenetic analysis focused on sequences from a Gag fragment from 32 isolates and on SU sequences from four isolates. Finally, higher diversity for this subtype was further supported by two recent studies. Examination of both Gag and envelope sequences derived from FIV subtype B isolates in Austria, where subtype B predominates, revealed subtype B clusters with sufficient genetic diversity to support their designation as subclades. ${ }^{146}$ A second study characterized a cluster of FIV isolates from Texas more closely related to FIV subtype B viruses than to other subtypes, yet clearly distinct from this subtype ${ }^{151}$ Findings from this study suggested that this virus cluster was either a separate subtype that recently emerged from subtype B or represented a subgroup within subtype B. Collectively, these findings strengthen the hypothesis of FIV subtype B as an older virus existing within the domestic cat population for a longer time period than other FIV subtypes. Host adaptation and lower virulence has been suggested to be associated with FIV B subtype isolates but not confirmed by rigorous examination.

Several reports have also presented evidence of both naturally occurring ${ }^{149,150}$ and experimentally induced ${ }^{152}$ intersubtype recombinant viruses derived from recombination within the env gene. Observation of intersubtype recombinants involving a common break-point within gag was also recently reported. ${ }^{153}$ These findings for FIV agree with reports of recombinant HIV-1 isolates generated by divergent HIV subtypes in geographic regions where multiple HIV subtypes are found ${ }^{154-156}$ Identification of multiple subtypes, significant divergence within the major subtype $\mathrm{B}$ viruses, and confirmation of naturally occurring FIV recombinants are relevant issues for FIV vaccine design and suggest that vaccines specific to geographic area and subtype prevalence may be required. Similar issues exist for HIV-1 vaccine design and provide an opportunity for the FIV 
vaccine model to address the importance of multisubtype vaccine approaches.

\section{Regulatory and Accessory Genes}

Only one regulatory gene ( $r e v$ ) and two accessory genes (vif and orfA) have been characterized for the FIV genome. Although several research groups have shown that FIV Rev and FIV Vif provide functions in virus replication similar to those described for analogous HIV-1 proteins, exhaustive examination of these FIV gene products has not been reported. FIV accessory genes, that are clearly analogous to primate lentivirus regulatory and accessory genes, including tat, vpr, vpu, vpx, or nef, have not been identified. Early reports proposed that FIV Orf-A encoded a HIV-1 Tat gene product. However, studies testing Orf-A transactivation of the FIV LTR did not demonstrate Orf-A activity comparable to HIV-1 Tat. ${ }^{39,40,43,61}$ Recent findings suggest that FIV Orf-A may express functions more similar to those of HIV-1 Vpr or perhaps Vpu, although data supporting this premise are very limited. ${ }^{157,158}$ However, the possibility that viral functions and activities expressed by multiple unique HIV-1 accessory proteins may be encompassed by a single FIV accessory protein along with specific domains within FIV structural proteins warrants further examination.

Genomes of all members of lentivirus subfamily encode for a HIV-1 Rev-like posttranscriptional regulatory protein that is expressed early in the viral life cycle from a multiply spliced mRNA species containing either orf$A$ and the $\mathrm{L}$ region of $e n v$ and orf- $H$, or the $\mathrm{L}$ region of $e n v^{42,66}$ and $\operatorname{orf}-H$ only. ${ }^{68}$ FIV Rev is a $23 \mathrm{kD}$ protein that is expressed from two exons derived from the $\mathrm{L}$ region of $e n v$ and from orf- $H$ (Figure 2). The first rev exon extends through the $5^{\prime}$ terminal $\mathrm{L}$ region of the $e n v$ gene and is in the same open reading frame as $e n v$. The second exon (orf- $H$ ) is located at the $3^{\prime}$ terminus of the env gene and overlaps the $5^{\prime}$ terminus of the $3^{\prime}$ LTR. Similar to HIV-1 Rev, FIV Rev is a nucleolar protein that binds as multimers to the cis-acting regulatory RRE sequence contained within unspliced and singly spliced viral mRNAs to promote nuclear export of these mRNA species to the cytoplasm and to also increase mRNA stability and protein translation. ${ }^{41,68,69,159,160}$ FIV Rev contains a highly basic domain similar to that described for other lentivirus Rev proteins. This basic domain located in the second exon, most likely serves as an RRE binding domain, although studies confirming the function of this FIV Rev element have not been reported. Another viral mRNA containing only the second Rev exon (orf$H$ ) has also been identified and encodes for a truncated Rev protein designated $\mathrm{p} 15^{\text {rev }}{ }^{66} \mathrm{P} 15^{\text {rev }}$ contains the highly basic domain and effector domain of Rev but lacks the $\mathrm{N}$ terminus derived from the first exon. This truncated Rev protein exhibited Rev activity by in vitro assays but only $20 \%$ of the activity observed for wild type full-length FIV Rev. The significance 
of $\mathrm{p} 15^{\mathrm{rev}}$ in virus replication has not been characterized. Expression of FIV structural and enzymatic proteins, including Vif, is dependent on Rev expression and activity. Accordingly, the Rev regulatory system is essential for productive virus replication.

Although FIV Rev shares similar activities and functions with HIV-1 Rev, the FIV Rev effector (activation) domain located immediately downstream of the basic domain, contains a nuclear export signal (NES) that differs significantly in amino acid sequence from those described for Rev effector domains of primate and ungulate lentiviruses ${ }^{60,161,162}$ The FIV Rev effector domain/NES lacks organized hydrophobic residues (leucine-rich clusters) and a core tetramer, which are properties that define the HIV-1 Rev NES. ${ }^{163}$ Instead, the FIV Rev effector domain/NES is characterized by basic residues and dispersed leucine residues similar to that observed for equine infectious anemia virus (EIAV) Rev. However, both FIV and EIAV Rev effector domains can functionally replace the effector domain of HIV1 Rev, a finding that suggests FIV and HIV-1 Rev proteins utilize similar cellular pathways for their activity. ${ }^{162}$ Similarity between FIV Rev and HIV-1 Rev is further supported by studies demonstrating that nuclear export of FIV and EIAV Rev proteins, as well as Rev function, is inhibited by leptomycin B, a drug previously shown to block HIV-1 Rev NES interactions with CRM1 (exportin 1). ${ }^{161}$ This observation suggests that binding of CRM1 for nuclear export may be a property that FIV Rev shares with HIV-1 Rev, regardless of their dissimilar activation domains. Another functional property shared by the FIV and HIV-1 Rev regulatory systems involves an interaction with cellular eukaryotic initiation factor 5A (eIF-5A), which is also associated with Rev's nuclear export of unspliced and singly spliced viral mRNA. ${ }^{164-166}$ Biologically active eIF-5A was previously shown to be required for HIV-1 Rev function and to specifically facilitate binding of Rev/RRE complex to CRM1. ${ }^{167}$ Activation of eIF-5A requires synthesis of the unique amino acid hypusine, which is sustained by deoxyhypusine synthetase. ${ }^{168} \mathrm{~A}$ recent report revealed that 1,8-diaminooctane, an inhibitor of deoxyhypusine synthetase, significantly inhibited FIV replication and specifically restricted FIV Rev function. ${ }^{166}$ Taken together, these observations demonstrate similarities shared between HIV-1 and FIV Rev proteins and validate FIV Rev as a model for potential antiviral therapies that target HIV-1 Rev.

An accessory gene designated as vif is conserved among all the lentiviruses with the exception of EIAV ${ }^{73}$ and has been determined to encode a factor necessary for virion cell-free infectivity, although not required for virion production. ${ }^{169,170}$ FIV vif resides at the 3 ' terminus of the pol gene in the viral genome and is translated from a singly spliced mRNA $^{171}$ to express a 23-kD basic protein (Figure 2). The role of FIV Vif in viral infectivity for primary feline lymphocytes and macrophages has been clearly established. ${ }^{172-174}$ However, biochemical characterization of FIV Vif has been limited and restricted to one report describing nuclear 
localization for this FIV accessory protein. ${ }^{175}$ Studies regarding mechanisms of Vif function have so far focused instead on primate lentivirus Vif proteins. Early studies demonstrated that the replication phenotype of vifdeleted HIV mutants was cell type-dependent and specifically dependent of the cell producing the virion. ${ }^{176}$ Cell types, including primary lymphocytes and macrophages as well as specific T-cell lines, required the presence of HIV-1 Vif expression for production of infectious HIV and were designated as "nonpermissive" for replication of vif-deleted mutants of HIV-1. However, specific human T-cell lines designated as "permissive cells," including SupT-1 cells and Jurkat cells, supported productive virus replication for HIV-1 vif-deleted variants. Cell fusion experiments showed that heterokaryons formed between permissive and nonpermissive cells displayed the nonpermissive phenotype, suggesting that nonpermissive cells naturally express an antiviral activity that inhibits the replication of vifdeficient virus. ${ }^{177}$ Virions produced in nonpermissive cells in the absence of the vif gene are impaired for reverse transcription of genomic RNA and therefore fail to establish full-length proviruses after entry into a target cell. The small number of reports focused on FIV Vif to date have not revealed a feline cell line "permissive" for vif deletion mutants of FIV. This observation may be due in part to the very few established feline T-cell lines that are available and permissive for primary wild type isolates of FIV.

Recent reports have revealed major breakthroughs in the understanding of HIV-1 Vif functions and interactions with host cell proteins. Sheehy and colleagues first identified a cellular protein designated CEM15 as the "nonpermissive" cell factor responsible for production of noninfectious virus particles in the absence of HIV-1 Vif. ${ }^{178}$ CEM-15 was later identified as APOBEC3G, a cell protein closely related to APOBEC1 and a member of the cytidine deaminase family of nucleic acid-editing enzymes. Numerous studies have now established APOBEC3G as a cellular factor that exerts an antiviral effect by deamination of cytosines to uracils in singlestranded minus-strand DNA during reverse transcription, resulting in either degradation of newly synthesized minus-strand DNA or guanidine to adenine hypermutations in the final double-stranded proviral DNA product. ${ }^{179-181}$ In the absence of HIV-1 Vif, APOBEC3G is packaged into virions, allowing this cellular protein to exert its antiviral effect during reverse transcription after virion entry into a target cell. However, if present during virion assembly, HIV-1 Vif forms a complex with human APOBEC3G that targets the cellular factor for proteosomal degradation and thereby prevents virion encapsidation of APOBEC3G to facilitate particle infectivity. ${ }^{182-184}$ These advances in the elucidation of HIV-1 Vif function have generated new enthusiasm for designing antiviral therapeutics targeting Vif and Vif-APOBEC3G interactions. Recent studies have also confirmed that other primate lentivirus (SIV) Vif proteins also target APOBEC3G in simian cells, although these interactions appear to be species-specific for some of 
the simian species. Whether nonprimate lentivirus Vif proteins, including FIV Vif, function similarly by interacting with a species-specific APOBEC3G analog has yet to be determined. Evidence that supports this possibility is derived from reports demonstrating that APOBEC3G degradation is mediated by the HIV-1 Vif SLQ(Y/F)LA domain, an amino acid motif that is conserved among all lentivirus Vif proteins including FIV Vif. ${ }^{173,182,183}$

FIV gene orf- $A$ (also referred to as orf-2) encodes a 77 amino acid accessory protein previously implicated to encode a Tat-like protein and is critical for efficient viral replication in peripheral blood mononuclear cells (PBMC) in vitro and in vivo. ${ }^{61,157,185-188}$ Although the orf- $A$ gene product contains a cysteine-rich domain within its $3^{\prime}$ terminus, similar to Tat proteins encoded by ungulate lentiviruses, this gene product does not include core and basic domains comparable to those found in Tat proteins encoded by primate lentiviruses, EIAV, or BIV. Amino acid sequence alignments of FIV Orf-A with visna virus and CAEV Tat proteins reveal a similar organization of conserved putative domains, including N-terminal hydrophobic, central leucine-rich, and C-terminal cysteine-rich regions. ${ }^{60}$ In addition, FIV Orf-A encodes previously unrecognized conserved tryptophans at positions 43 and 66 positioned similarly to conserved tryptophan residues 63 and 85 of visna virus Tat. Two recent studies demonstrated a moderate upregulation of the FIV LTR promoter activity by coexpression of Orf-A in transient reporter gene expression assays. ${ }^{66,67}$ In contrast, earlier reports revealed either a small effect or no effect imposed by Orf-A on FIV LTR-directed gene expression. ${ }^{39,40,43,61}$ These findings suggest that Orf-A very likely drives FIV-LTR-directed transcription, but by indirect mechanisms involving interactions with cellular transcription factors.

A recent report revealed Orf-A to be important in the late steps of the FIV life cycle involved in virion formation and in early steps involved in virus infectivity and mapped critical Orf-A domains needed for these steps in replication. ${ }^{157}$ Central leucine-rich and C-terminal cysteine-rich regions, along with a conserved central tryptophan (residue 43) within Orf-A, were shown to be critical determinants for efficient virus replication and infectivity. The leucine-rich domain was important for infectivity, whereas tryptophan 43 and the cysteine-rich domain were important for both infectivity and virion formation. Importantly, deletions and point mutations in orf-A imposed a small or no effect on FIV LTR-driven viral gene expression and no effect on viral protein expression. These findings suggested that orf- $A$ represents a FIV-encoded analog more similar to accessory genes $v p r, v p u$, or nef rather than the regulatory tat gene encoded by the primate lentiviruses. This concept was further supported by another recent study using mammalian expression plasmids encoding wild type or deletion mutant Orf-A proteins fused to the $\mathrm{C}^{\prime}$-terminus of green fluorescent protein (GFP) to evaluate Orf-A subcellular localization and effects on cell function. ${ }^{158}$ Findings from this study demonstrated nuclear localization 
for the GFP-Orf-A fusion protein and allowed mapping of a NLS (residues 44-54) critical for nuclear import of FIV Orf-A. Furthermore, assessment of cell cycle profiles of cells transiently expressing GFP-Orf-A revealed that Orf-A causes an arrest at G2 of the cell cycle. These novel findings suggested that Orf-A is a nuclear protein that expresses some properties similar to those reported for HIV-1-encoded Vpr. Collective data generated from both reports suggest the possibility that Orf-A may encode specific functions attributed to several different HIV-1 encoded accessory protein but not all functions characterized for a single HIV-1 accessory protein. Additional support for this possibility is provided by a recent report showing that CAEV Tat, which shares structural homology with FIV Orf-A, also localizes to the nucleus and arrest cells in G2. ${ }^{189}$ Verification of these findings for FIV Orf-A from other research groups and for Orf-A proteins encoded by multiple FIV isolates will be needed to confirm the similarities between FIV Orf-A and HIV-1 Vpr and other HIV-1 accessory proteins.

\section{FIV TROPISM}

\section{FIV Receptor Usage}

Natural and experimental infection of cats with biological and molecularly cloned isolates of FIV consistently induce an acute viremia associated with T-cell alterations including depressed CD4: CD8 T-cell ratios and CD4 T-cell depletion. ${ }^{4-7,10,11,21,190-194}$ Early studies revealed that targets for FIV in vitro and in vivo included CD4 T-cells, macrophages, dendritic cells, microglia, and astrocytes similar to those for HIV infection in humans, but also included CD8 T-cells, and B-cells (Table II). ${ }^{4,18,23,186,195-210}$ Early reports also demonstrated that continuous passage of particular FIV isolates in cell culture selected for virus variants capable of replication in feline adherent cell lines, including Crandell feline kidney cells (CrFK) and G355-5 cells, as well as established feline interleukin (IL)-2-independent T-cell lines. ${ }^{2,25,42,211}$ Importantly, experimental inoculation studies in cats revealed that cell culture-adapted viruses represented a particular subset of viral variants that exhibited reduced replication and virulence in vivo. ${ }^{111,192,197,212}$

FIV infection of feline CD4-negative adherent cell lines provided indirect evidence that FIV differs with HIV-1 and does not utilize CD4 as a primary receptor. In addition, direct evidence refuting FIV usage of CD4 was provided by studies revealing an absence of virus infectivity for nonlymphoid cells expressing feline CD4. ${ }^{213}$ Subsequent reports described blocking of FIV infectivity by an antibody specific for feline CD9, a cell surface antigen belonging to the four-transmembrane-spanning domain superfamily (TM4SF) ${ }^{214,215}$ However, anti-CD9 antibody was later shown to 
TABLE II

Comparison of FIV and HIV-1 Replication Properties In Vitro

\begin{tabular}{|c|c|c|}
\hline Property & FIV & HIV-1 \\
\hline $\begin{array}{l}\text { Permissive } \\
\text { primary cells }\end{array}$ & $\begin{array}{l}\text { PBMC, CD4 T-cells, CD8 T-cells, } \\
\text { macrophages, various other } \\
\text { cell types including microglia } \\
\text { and astrocytes }\end{array}$ & $\begin{array}{l}\text { PBMC, CD4 T-cells, macrophages } \\
\text { various other cell types } \\
\text { including microglia and } \\
\text { astrocytes }\end{array}$ \\
\hline $\begin{array}{l}\text { Permissive } \\
\text { cell lines }\end{array}$ & $\begin{array}{l}\text { IL-2-dependent T-cell lines } \\
\text { IL-2-independent T-cell lines } \\
\text { fibroblastic adherent cell } \\
\text { lines (CrFK and G355-5) }\end{array}$ & $\begin{array}{l}\text { Various CD4 T-cell lines } \\
\text { macrophage cell lines }\end{array}$ \\
\hline Cytopathic effects & $\begin{array}{l}\text { Syncytium, giant cell formation } \\
\text { cell lysis, apoptosis }\end{array}$ & Same as FIV \\
\hline \multicolumn{3}{|l|}{ Receptors } \\
\hline Primary isolates & CD134 and CXCR4 & $\begin{array}{l}\text { CD4 and CCR5 or dual-tropic } \\
\text { (R5X4) }\end{array}$ \\
\hline Adapted isolates & $\begin{array}{l}\text { CD134 and CXCR4 or } \\
\text { CXCR4 only }\end{array}$ & $\begin{array}{l}\text { CD4 and CXCR4 or other } \\
\text { "orphan" chemokine receptors } \\
\text { or CXCR4 only }\end{array}$ \\
\hline Host range & Feline cells only (?) & $\begin{array}{l}\text { Human, some nonhuman primate } \\
\text { cells }\end{array}$ \\
\hline $\begin{array}{l}\text { Viral tropism } \\
\text { determinants }\end{array}$ & $\begin{array}{l}\text { Env: V3 region and TM } \\
\text { Orf-A U3 domain of LTR }\end{array}$ & $\begin{array}{l}\text { Env: V3, V1/V2 } \\
\text { Vpr }\end{array}$ \\
\hline
\end{tabular}

block virus infection by inhibition of virus release and not by interference with virus-receptor binding. ${ }^{216}$

A major breakthrough in characterization of FIV cell surface receptors resulted from observations showing that cell culture-adapted FIV isolates primarily utilize the $\beta$-chemokine receptor CXCR4 for infection in a similar fashion to T-cell line-adapted isolates of HIV-1 (Table II). ${ }^{33,217}$ Induced cell surface expression of CXCR4 was shown to mediate a susceptibility to FIV infection ${ }^{33,218}$ that could be abrogated by treatment with natural ligands for CXCR4 such as stromal cell-derived factor (SDF-1) and with CXCR4 antagonists including AMD3100. ${ }^{219-221}$ Furthermore, critical determinants for FIV infection were mapped to the extracellular loop of CXCR4. ${ }^{218,222}$ Multiple reports have now confirmed that cell cultureadapted FIV isolates are capable of using CXCR4 exclusively for virus entry and infection. ${ }^{33,36,218-221,223,224}$

Results of subsequent reports suggesting FIV usage of chemokine receptors CCR5 and CCR $3^{223,225}$ have conflicted with observations of other investigators and may have resulted from enhanced expression of CXCR4 associated with ectopic expression of CCR5. ${ }^{224}$ Use of other chemokine receptors by particular FIV isolates is not clear at this time and is under investigation. Similar to HIV-1 envelope proteins, binding of FIV Env to 
other cell surface makers, including heparan sulfate proteoglycans (HSPGs) and DC-SIGN, has been shown and is specific for particular isolates. ${ }^{35,36}$ Binding of DC-SIGN was observed with recombinant Env proteins derived from primary and cell culture-passaged FIV isolates, whereas binding of HSPGs was observed only with a cell culture-passaged FIV strain. Although virus entry was shown to be mediated exclusively by CXCR4, virus infectivity was enhanced by factors including temperature or HSPGs, which either increase concentration of or binding for CXCR4, respectively. ${ }^{226}$ The roles of binding of DC-SIGN and HSPGs by FIV Env in FIV infection and pathogenesis in vivo are currently not well understood and warrant further examination.

Previous studies confirming CXCR4 as a receptor also revealed that CXCR4 expression was not sufficient for infectivity of primary FIV isolates and suggested the likehood that primary isolates required a second receptor for binding and entry. One report describing coimmunoprecipitation of a recombinant FIV envelope protein with a $40 \mathrm{kD}$ cellular protein provided further evidence of a non-CXCR4 receptor for FIV. ${ }^{36}$ A second major advance in FIV receptor biology was the recent identification of this cellular protein as CD134, a $43 \mathrm{kD}$ cell surface marker, and the receptor utilized by primary FIV isolates in conjunction with CXCR4. ${ }^{32,227}$ CD134 is a member of the tumor necrosis factor-receptor superfamily and is expressed primarily on activated CD4 T-cells (mouse and human) after T-cell receptor (TCR) engagement. ${ }^{228} \mathrm{~A}$ recent report confirmed a similar phenotype for feline CD134 by showing up-regulation of CD134 expression on mitogen-activated feline CD4 T-cells. ${ }^{226}$ Binding of cell surface CD134 with cellular CD134 ligands provides a costimulatory signal that results in proinflammatory effects, as well as proliferation, migration, and cytokine production by memory T-cells. Low level CD134 expression has also been reported for activated CD8 T-cells, activated B-cells, and macrophages in mice and humans ${ }^{229-231}$ but has not been confirmed for the same feline cell lineages.

So far, preliminary reports indicate that infection by primary isolates requires binding of both CD134 and CXCR4 ${ }^{32,227}$ Assessment of receptor usage by larger panels of primary FIV isolates will be needed to determine if additional chemokine receptors may also be used for FIV infection, as previously observed for HIV-1, and may be associated with specific FIV replication/pathogenic phenotypes. Whether infection of CD8 T-cells, B-cells, and macrophages previously observed in later stages of FIV infection is due to CD134-independent usage of CXCR4 expressed on these cell populations, or results from usage of both CD134 and CXCR4, has not yet been clarified. Clearly, usage of CD134 as a receptor by primary FIV isolates explains the specific targeting of the $\mathrm{CD} 4 \mathrm{~T}$-cell population for depletion observed with FIV infection and the similarities in the acquired immunodeficiencies induced by both HIV-1 and FIV. Furthermore, these obser- 
vations elucidate a mechanism by which FIV and HIV-1 utilize unique receptors (CD4 and CD134) to target a similar subset of T-cells (i.e., activated and resting memory CD4 T-cells) to impart disease pathogenesis. ${ }^{232-237}$ Accordingly, these findings further validate the importance of FIV infection as a model for examination of HIV-1-induced AIDS. Examination of pathogenic effects and cellular dysfunction imposed by virus-binding of CD134 expressed on CD4 T-cells in vitro will also be important for defining potential viral mechanisms for induction of feline acquired immunodeficiency in vivo.

\section{Viral Determinants for Cell Tropism}

Replication phenotypes in vitro and in vivo clearly distinguish cell culture-adapted FIV isolates from primary isolates. Most primary FIV isolates examined to date require both CD134 and CXCR4 for infection and exhibit a cell tropism restricted to primary feline PBMC, selected IL-2dependent T-cell lines, and possibly primary macrophages and astrocytes, ${ }^{2,42,61,196-199,238}$ although testing for tropism to the latter two cell types has been infrequently reported (Table II). In contrast, FIV variants passaged in vitro utilize CXCR4 solely for efficient infection and replication in feline adherent cell lines including CrFK cells, ${ }^{33,36,218-221,223,224}$ as well as feline PBMC, IL-2-dependent and independent feline T-cell lines and macrophages. ${ }^{2,42,61,187,190,197,211,239-242}$ These highly passaged isolates, however, exhibit severely restricted virus replication and pathogenicity in vivo as discussed above. ${ }^{11,192,197,212}$ Multiple studies using FIV molecular clones have mapped Env as a major determinant that expands cell tropism to include feline adherent cell lines including CrFK cells and G355-5 cells (feline glial cell line), as well as feline astrocytes (Table II) ${ }^{33,212,223,243-248}$ Similar to the V3 domain of HIV-1 Env, the V3 domain of the FIV SU encodes specific tropism determinants ${ }^{212,223,243,244,249-251}$ in addition to important neutralizing antibody epitopes (Figure 3). ${ }^{252-254}$ Critical amino acid residues mapped within the FIV Env V3 domain appear to determine adherent cell tropism by modifying the overall charge of the V3 loop. ${ }^{22,243,244}$ A recent report describing the evolution of a nonpathogenic FIV isolate to a more pathogenic virus in vivo over time suggested that mutation $\mathrm{E} 409 \mathrm{~K}$ within the V3 loop not only imposed CrFK cell tropism but also contributed to virus attenuation. ${ }^{212}$ This hypothesis is supported by the consistent observation of severely restricted replication in vivo observed for viruses exhibiting a tropism for CrFK cells in vitro. ${ }^{111,192,197,212}$ Other Env determinants affecting FIV cell tropism for adherent cells have been mapped to the second constant domain within SU, the extracellular domain of $\mathrm{TM},{ }^{247}$ and the cytoplasmic domain of TM. ${ }^{245}$ Although V3 and V4 domains of FIV SU are also reported to influence FIV macrophage and brain microglia tropism, ${ }^{209,255}$ viral determinants critical for macrophage tropism as well as the frequency 
of macrophage tropism across many primary isolates have not been well examined for FIV. Mechanisms by which these important Env residues or domains contribute to virus infectivity (for all cell types) either by effects on Env conformation or binding affinity to either CXCR4 or CD134 are also not well understood at this time and warrant investigation to further characterize the FIV animal model.

Multiple reports confirmed that the gene product of accessory gene orf- $A$ is a critical determinant for infection of feline $\mathrm{PBMC}^{61,157,187,197}$ but is not required for infection of adherent cell lines including CrFK cells. However, the role of Orf-A in FIV tropism for feline macrophages has received only limited study and is unclear based on conflicting data reported from different studies. ${ }^{61,187,197}$ Mechanisms by which Orf-A impacts virus infectivity of PBMC are also undetermined at this time.

Interestingly, early studies suggested that restriction of productive FIV infection in human cells resulted primarily from a block in FIV transcription rather than a block in virus entry. ${ }^{40,256-259}$ The capability of FIV isolates to establish latent infections in human cells $^{258}$ was later explained by demonstration of cell culture adapted-FIV usage of human CXCR4 for fusion and infection of human cells. ${ }^{33}$ Subsequent studies focused on FIV vector development revealed that the FIV U3 domain was the determinant responsible for restricted virus replication in human cells and showed that replacement of U3 with the cytomegalovirus immediate early promoter produced FIV vectors capable of viral gene expression in human cells. ${ }^{225,260}$ Studies reported by one research group showing that FIV is capable of productive infection of primate cells both in vitro and in vivo ${ }^{261,262}$ have not been confirmed by other independent researchers but suggest FIV infection of nonfeline cells may warrant further examination.

\section{EPIDEMIOLOGY AND TRANSMISSION}

Similar to other lentiviruses, the presence of FIV-specific antibodies signifies an established virus infection that will persist throughout the remaining lifetime of the host. Antibody detection ELISAs and other immunochromatographic methods (excluding western blot) have served as the primary screening diagnostic assays for FIV infection in clinical veterinary practice and epidemiologic studies. ${ }^{263-266}$ Although most FIVinfected cats produce antibodies to both Gag and Env structural proteins, a small proportion of cats will test positive for antibodies specific to only one of these two structural proteins. ${ }^{267}$ Therefore, currently available commercial FIV antibody assays include both FIV Gag and Env recombinant antigens for optimal sensitivity as well as specificity. ${ }^{264}$ Current and earlier serologic studies have shown that FIV is enzootic worldwide. ${ }^{13,15,264,266,268-273}$ Evaluation of mutations within virus subtypes suggests that FIV has been 
present in domestic cats for a significant period of time, especially when compared to the relative short evolution of HIV. ${ }^{142,150}$ FIV prevalence varies greatly depending on geographic location and other variables of the tested cat populations. Among clinically healthy cats, the prevalence of FIV may be as low as $1 \%$ or less as observed in central European countries and the United States, or as high as $30 \%$ as reported in Japan and Australia. ${ }^{14,273}$ The seroprevalence rates in sick cats appear to be several times higher than those in their healthy counterparts and reflect the disease-inducing potential of FIV. ${ }^{13,15}$ Age and gender also markedly affect FIV prevalence. Most infections are acquired after one year of age, and prevalence increases up to approximately 10 years of age and then remains stable or tends to decline as the mean lifespan of a domestic cat is about 15 years. ${ }^{274}$ Viruses similar to FIV have been documented in several nondomestic felids such as lions, panthers, and bobcats. ${ }^{269,275-282}$ The large genetic diversity observed for lentiviruses among different nondomestic felids and between nondomestic feline lentiviruses and FIV, however, do not support the likelihood that nondomestic feline lentiviruses contribute significantly to the circulation of FIV in domestic cats. There is little evidence that FIV is transmissible to any other species including humans ${ }^{13}$ with the exception of a recent single report describing experimental FIV infection of nonhuman primates (cynomolgus macaques). ${ }^{262}$

Precise modes of natural FIV transmission among domestic cats are not yet clear. Nevertheless, strong epidemiologic evidence implies that biting and fighting may be the predominant route of transmission (Table III). The importance of this route of transmission is corroborated by observations of the highest prevalence of infection in rural feral cat populations and urban areas containing a high density of freely roaming cats, as in Japan, and a higher prevalence of infection in adult tomcats. ${ }^{13,15,270,273,283-287,288}$ In fact, one epidemiologic study revealed that a pattern of increased FIV infection in orange cats compared to nonorange cats correlated with the pattern of more aggressive behavior also exhibited by orange cats. ${ }^{289}$ In addition, evidence of virus in saliva harvested from infected cats ${ }^{290}$ and an infection experiment involving virus inoculation by simulating biting further substantiate that transmission by this route is highly effective. ${ }^{13}$

Early epidemiologic and experimental infection studies refuted the possibility of naturally occurring vertical transmission of FIV. ${ }^{2,13,291}$ However, later experimental inoculation studies confirmed virus transmission to newborn kittens from queens either acutely or chronically infected with different FIV isolates. ${ }^{19,20,292-294}$ Such transmission appeared to occur via in utero $^{294}$ and postnatal routes,,$^{19,20,293,295}$ although intrapartum transmission has also been implicated. ${ }^{19}$ Interestingly, recent studies provided evidence of vertical transmission resulting in occult infection of kittens characterized by the presence of viral DNA in tissues in the absence of replicating 
TABLE III

Comparison of FIV and HIV-1 Infection Properties In Vivo

\begin{tabular}{|c|c|c|}
\hline Property & FIV & HIV-1 \\
\hline $\begin{array}{l}\text { Transmission } \\
\text { (natural) }\end{array}$ & Blood (bite wounds), vertical (?) & Blood, vertical, sexual \\
\hline $\begin{array}{l}\text { Transmission } \\
\text { (experimental) }\end{array}$ & $\begin{array}{l}\text { Blood (bite wounds), vertical, } \\
\text { vaginal and rectal mucosa }\end{array}$ & Not applicable \\
\hline Cell tropism & $\begin{array}{l}\text { CD4 T-cells, CD8 T-cells, B cells, } \\
\text { macrophages, dendritic cells, } \\
\text { microglia follicular dendritic } \\
\text { cells CD4+CD25+ T-cells, } \\
\text { thymocytes, megakaryocytes }\end{array}$ & $\begin{array}{l}\text { CD4 T-cells, macrophages } \\
\text { dendritic cells, microglia } \\
\text { follicular dendritic cells } \\
\text { thymocytes, CD8 T-cells and } \\
\text { others (?), CD4+CD25+ } \\
\text { T-cells (?) }\end{array}$ \\
\hline Tissue tropism & $\begin{array}{l}\text { Blood, lymphoid tissues, } \\
\text { gastrointestinal tract, CNS } \\
\text { genital tract, liver, kidney }\end{array}$ & $\begin{array}{l}\text { Blood, lymphoid tissues, } \\
\text { gastrointestinal tract, CNS } \\
\text { genital tract }\end{array}$ \\
\hline Host range & Restricted to felids & $\begin{array}{l}\text { Restricted to humans and } \\
\text { some nonhuman primates }\end{array}$ \\
\hline Immunopathology & $\begin{array}{l}\text { Lymphoid follicular hyperplasia } \\
\text { (early) and CD } 8 \alpha+\beta \text { low T-cell } \\
\text { subset expansion, followed by } \\
\text { lymphoid depletion, CD } 4 \\
\text { T-cell depletion, cytokine } \\
\text { dysregulation, and AIDS }\end{array}$ & Same as FIV \\
\hline
\end{tabular}

virus in peripheral blood, as well as absence of antiviral antibody apart from maternal antibody. ${ }^{206,296,297}$ Although these occult infections resulting from vertical transmission were considered regressive or transient as reported by one study, ${ }^{296}$ the duration of persistence of this type of infection has not been thoroughly examined. These covert or occult infections resemble previously described restricted FIV infections that were detectable only by PCR amplification of viral DNA from blood or tissues and that resulted either from inoculation with extremely attenuated viruses ${ }^{192}$ or from persistent but nontraumatic contact between naive and infected cats. ${ }^{298}$ Furthermore, similar sequestered virus infections in the absence of antibody have been reported for SIV infection ${ }^{299,300}$ and implicated in particular human populations at high risk for HIV infection. ${ }^{301,302}$ Collectively, these findings suggest the possibility that the incidence of natural FIV infection by vertical transmission may be underestimated, since diagnostic assays focus on antibody or virus in peripheral blood only.

Detection of virus in semen of infected cats has been observed ${ }^{303,304}$ and experimental infection by artificial insemination has also been reported. ${ }^{305,306}$ Numerous experimental studies have shown the feasibility of infection by the vaginal route $\mathrm{r}^{16-18,21-23,307-311}$ and demonstrated the utility 
of this feline animal model for examination of mucosal transmission and viral pathogenesis. Although these observations and experimental studies suggest the possibility of sexual transmission of FIV, definitive observations of natural transmission by this route so far have not been reported.

\section{VIRUS INFECTION AND HOST RESPONSES}

\section{Virus Infection in Cell Culture}

Primary feline PBMC activated by concanavalin A (ConA) and specific feline IL-2-dependent T-cell lines, including FET-1 cells, ${ }^{242}$ MYA-1 cells, ${ }^{312}$ FCD4E cells, ${ }^{201}$ 104C-1 cells, ${ }^{227}$ and MCH5-4 cells, ${ }^{211}$ have proven to be highly permissive for propagation of biological and specific molecularly cloned isolates of FIV. As described in the section discussing FIV receptor usage, productive virus replication in these cell types results from expression of feline CXCR4 and CD134 cell surface molecules that has been confirmed, at least for activated feline PBMC and 104C-1 cells (Table II). ${ }^{32,227}$ Established feline adherent cell lines, including CrFK cells and G355-5 cells, as well as feline IL-2-independent lymphoid cell lines such as 3201 cells, MCH5-4DL, and 104-C1DL, have been used for propagation of CD134-independent isolates. ${ }^{13,25,42,211}$ Feline IL-2-independent lymphoid cell lines (FL-4 cells) chronically infected with FIV isolate FIV-Petaluma were also generated for production of virus for use in whole killed virus vaccines and diagnostics. ${ }^{242}$

Assays used to confirm virus replication and production in vitro have included FIV p24Gag antigen capture ELISAs, RT assays, indirect immunofluorescence or immunocytochemical assays for viral structural proteins, and PCR assays for viral nucleic acids. Quantitative real-time PCR assays for both FIV RNA and DNA have been developed for quantitation of viral nucleic acids of different isolates in either cell culture supernatants, plasma, or cells. ${ }^{11,157,190,212,309,313-316}$ The appearance of virus-induced cytopathic effects (CPE) consisting of syncytium, giant cell formation, and cell lysis (Table II) in feline PBMC and T-cell lines may be PBMC donor and FIV isolate-dependent and has proven less dependable for use as a marker of virus infection in these cell types. ${ }^{1,212,317,318}$ In contrast, infection of adherent cell lines CrFK and G355-5 and IL-2-independent lymphoid cells lines (3201, MCH5-4DL, 104-C1DL) with CD134-independent FIV isolates may result in prolific CPE that is most consistently observed in CrFK cells and which may serve as a indicator of infection for these isolates. ${ }^{13,32,211,238,241,242,248}$ CD134-independent FIV strains have been selected by extensive cell culture passage of biological isolates and mimic CD4independent HIV-1 isolates that are also extremely cytopathic and efficient for replication in established T-cell lines in vitro. ${ }^{73}$ 


\section{Virus Localization in the Host}

Most knowledge regarding acute FIV infection and virus localization and dissemination has been derived from experimental inoculation studies using both biological and molecularly cloned FIV isolates. Factors that may affect virus localization and distribution during the acute phase of infection are virus pathogenicity, virus tropism, titer of virus inocula, presence of virus-infected cells in the inocula, route used for virus infection, and age of host. Experimental studies testing highly pathogenic and attenuated FIV isolates, viruses inoculated by parenteral or mucosal routes, and cell-free or cell-associated virus inocula have been reported. However, careful examination of effects imposed by each of these variables on viral distribution and ultimate disease, as well as mechanisms for these effects, has yet to be described. Regardless, reports so far describe a virus distribution pattern for pathogenic FIV isolates in vivo that is similar to that reported for HIV1 (Table III). . $^{319-321}$

Early published experimental infection studies designed to examine FIV localization in vivo used either intraperitoneal, intravenous, intramuscular, $4,7,186,200,201,322-324$ intrathecal, or bone marrow inoculation ${ }^{198}$ of specific pathogen-free cats with various FIV isolates. Blood and tissues harvested from infected cats were assayed for infection by virus isolation from PBMC, viral nucleic acid detection by PCR or in situ hybridization, or viral antigen detection by immunocytochemical analyses. Later reports described experimental studies testing virus localization after virus mucosal delivery by either vaginal, rectal, or oral/nasal routes, ${ }^{16,18,21,23,307,309,325}$ in addition to either intraperitoneal or intravenous routes ${ }^{193,197,202,207,326-328}$ or intracranial injection. ${ }^{329,330}$ Virus localization in systemic lymphoid and central nervous system (CNS) tissues after FIV proviral DNA inoculation has also been examined. ${ }^{331,332}$ Collectively, these studies revealed localization of virus in PBMC and plasma, peripheral, and systemic lymphoid tissues, small and large intestinal tracts, and CNS tissues within 10 to 21 days after inoculation of the host, regardless of route of infection tested. In fact, one report described virus detection in gastrointestinal mucosa and associated lymphoid tissue by 1 to 3 days after oral/nasal infection and rapid dissemination of virus to systemic lymphoid tissues, bone marrow, and PBMC within 7 to 10 days after infection. ${ }^{23}$ Similarly, virus was detected in vaginal mucosa and spleen within 3 days after vaginal delivery of virus. ${ }^{23}$ However, virus infection of vaginal mucosa has been examined in very few studies and was observed only in those testing vaginal delivery of specific virus isolates (FIVB-2542 and FIV-PPR) ${ }^{16,23,309}$ and was not observed after vaginal infection with other FIV isolates ${ }^{18,23}$ or after intravenous virus infection. ${ }^{207}$ Similar to observations for the SIV animal model, ${ }^{299,333}$ experimental FIV infection by mucosal routes generally required higher titered virus inocula than 
required by parenteral injection, ${ }^{16,309}$ and one study reported a higher efficiency for infection with cell-free virus inocula compared to cell-associated virus. ${ }^{334}$ Virus has also been detected in other nonlymphoid organs including liver and kidney. ${ }^{4,200,207,332}$ Importantly, FIV infection of the CNS has been well documented ${ }^{4,198,200,202,255,294,324,335,336}$ and utilized as an animal model for HIV-1-induced neurologic deficits. ${ }^{210,324,329,330,332,337-344}$

Kinetics of virus emergence in blood and individual tissues has varied depending on the route of inoculation, virus strain, and infectious titer of virus inocula. Virus load in peripheral blood based on either virus isolation from PBMC or plasma viral RNA quantitation, may peak anywhere from 14 to 56 days after experimental infection. ${ }^{4,11,21,191,200}$ Significant virus loads during the acute stage of infection may also be observed in both peripheral and systemic lymph nodes, gastrointestinal tissues (predominantly submucosa and lamina propria), spleen, thymus, and bone marrow $^{4,23,185,193,200,207}$ and will precede the appearance of peak viremia in peripheral blood. These findings generally mimic virus distribution during acute infection for both HIV-1 and pathogenic SIV isolates. ${ }^{319,321,345}$ It is important to note the similar robust virus replication in gastrointestinal mucosal lymphoid tissue (GALT) observed during early time points of FIV, ${ }^{4,200,207}$ SIV, ${ }^{346,347}$ and HIV-1 ${ }^{348-350}$ infection. Although virus has been detected in the CNS during both acute and chronic phases of infection, quantitative data describing virus load in specific CNS tissues has been scarce. Virus loads in the PBMC and plasma generally decrease to lower set points during chronic asymptomatic infection, although this finding may be variable and dependent on virus strain pathogenicity ${ }^{17,212}$ and has not been as well defined for experimental FIV infection as reported for experimental SIV infection of rhesus macaques. FIV loads in individual tissues at sequential time points spanning acute, chronic asymptomatic, and terminal AIDS stages of disease also have not been well examined.

Similar to findings for HIV-1 and SIV infection, ${ }^{319-321,345,351}$ cellular subsets targeted during the acute stage of FIV infection have included CD4 T-cells, monocytes, macrophages, mucosal dendritic cells, mature and immature thymocytes, brain microglia and lymph node follicular dendritic cells (FDC) (Table III). 4,193,194,197,199,201-204,207,208,307,327,338,352,353 As discussed in a previous section of this chapter, FIV differs from HIV-1 by exhibiting a broader tropism in vivo that also includes megakaryocytes, ${ }^{323}$ CD8 T-cells and B-cells, ${ }^{186,193,197,201,307}$ although a few reports have described either HIV1 or SIV infection of CD8 T-cells. ${ }^{354,355}$ The variation in observations from different reports regarding the frequency and stage of infection for which virus or viral nucleic acid are detected in macrophages, CD8 T-cells, and B-cells may relate to differences in virus isolates, routes used for virus infection, age of the host, and virus detection assays. However, several studies report that either T-cells, or specifically CD4 T-cells, in both blood and 
tissues, are the predominant cell type harboring virus during the acute stage of infection. Virus load in CD4 T-cell populations decrease over time as the host progresses into chronic infection, ${ }^{4,194,201,207,208,307,352}$ while virus loads in macrophages and B-cells appear to increase. The relationship of stage of infection to virus load in other specific cell populations, including CD8 T-cells, mucosal dendritic cells, lymph node follicular dendritic cells, thymocytes, cells within the CNS, and other nonlymphoid tissues, has received little examination so far, although one report revealed a decrease in FIV-infected CD8 T-cells in lymphoid tissues by 10 weeks after infection. $^{307}$ A recent report described FIV infection in vivo of CD4+CD25+ T-cells, a cell population reminiscent of immunosuppressive CD4 T regulatory (Treg) cells. ${ }^{356}$ This $\mathrm{CD} 4+\mathrm{CD} 25+$ Treg cell population isolated from FIV-infected cats was shown in another recent report to coexpress costimulatory molecules B7.1, B7.2, and CTLA4 and to be anergic and resistant to clonal deletion. ${ }^{357}$ These findings warrant further investigation and suggest that CD4+CD25+ Treg cells may serve as an important long-lived reservoir for latent FIV in lymphoid tissues and currently are under examination as potential reservoirs for HIV-1 infection in vivo.

\section{Clinical Disease}

FIV infection results in progressive impairment of the immune system, including loss of CD4 T-cells, inverted CD4:CD8 ratios, heightened susceptibility to infectious agents, disruption of immune cell function, and deterioration of major lymphoid tissues and organs of the hosts. ${ }^{14,17,358}$ Observations from studies involving either experimental or natural infections, ${ }^{2,7-9,13,359-363}$ show that FIV disease course is very similar to that induced by HIV-1 infection and can be similarly divided into four to five stages based on type and severity of the clinical signs of infection. These stages of infection have been described as acute or primary, chronic asymptomatic, persistent generalized lymphadenpathy (PGL), AIDS-related complex (ARC), and feline AIDS (FAIDS). Notably, FIV and HIV-1 infection are usually associated with a prolonged asymptomatic phase that can last 10 years or more for infected humans and cats, and which constitutes most of the lifetime of the infected cat. ${ }^{360,364}$ In contrast, pathogenic SIV isolates induce an accelerated progression of immunodeficiency, resulting in death within 2 years of infection of rhesus macaques with a relatively short or absent asymptomatic phase of infection. ${ }^{365,366}$

The acute phase begins 1 to 4 weeks after FIV infection and may span a time period of 2 to 6 months. This stage of infection is characterized by a transient peak in peripheral blood (plasma and PBMC) virus load that is accompanied by a precipitous decline in CD4 T-cell counts and CD4:CD8 T-cell ratios. Depending on pathogenicity of virus isolate and age of the host, clinical and hematological abnormalities may include generalized 
lymphadenopathy, mild pyrexia, dullness, depression, anorexia, and neutropenia. Similar to findings for HIV-1 infection, FIV pathogenicity and clinical prognosis correlate to virus replication and load and to severity of clinical signs and hematologic deficits exhibited during the acute phase of infection. ${ }^{11,21,185,191,192,212,313,367,368}$ Another property shared by FIV and HIV-1 acute infection ${ }^{369,370}$ relates to the effect of age of the host on severity of clinical and hematologic disease at the time of infection. Multiple studies have shown that severity of primary phase FIV-induced disease was increased for neonatal kittens experimentally infected at birth. ${ }^{190,318,371-374}$

Primary FIV infection recedes as the host generates virus-specific immune responses and as virus loads decrease. Concordant with emergence of antiviral immune responses and reduced peripheral blood virus loads is an increase in CD4 T-cell count and CD4:CD8 T-cell ratio, although peripheral blood CD4 T-cells counts do not usually return to preinfection concentrations. The infected cat enters a relatively asymptomatic phase of FIV infection where control of virus load by host immune responses is presumed and may last for 5 to 6 years or for a significant proportion of the remaining life span of the cat. However, long-term observation of both experimentally and naturally infected cats has shown that peripheral blood CD4 T-cell counts slowly but progressively decrease during the asymptomatic phase and that clinical disease eventually becomes apparent. ${ }^{8,9}$

Careful observation of infected cats over time may reveal the reappearance of PGL as an early manifestation of clinical disease that is associated with vague signs of disease including recurrent fevers and weight loss. ${ }^{9,13-15}$ After the appearance of PGL and other vague clinical signs, infected cats generally progress into ARC, a phase characterized by the development of chronic persistent infections with pathogens that are usually self-limiting and involve the oral cavity, upper respiratory tract, ocular tissues, skin, and other body sites. Progression from ARC to FAIDS may be distinguished by infections with opportunistic pathogens, severe wasting, neoplastic disorders including non-T-cell lymphomas, neurologic disease, leukopenia, and anemia. Virus load increases and severe depletion of peripheral blood CD4 T-cells are observed. Survival time is usually less than a year after the onset of FAIDS.

\section{FIV Immunodeficiency and Pathology}

A gradual but progressive CD4 T-cell depletion that mimics primary immune deficiency observed in HIV-1 AIDS is the hallmark of immunodeficiency associated with both experimental and natural FIV infection in cats. ${ }^{5-8,10,11,21,185,190,192,193,200,212,361,375}$ CD4 T-cell subset depletion has been observed in peripheral blood and lymphoid tissues including thymus, ${ }^{22,193,374}$ during both early and chronic stages of infection (Table III). An increase in CD8 T-cell concentration is frequently associated with reduc- 
tion in CD4 T-cell counts and either contributes to, or largely accounts for, lower CD4/CD8 T-cell ratios observed during acute and chronic infection with either FIV or HIV-1. This elevation in the CD8 T-cell count is due to an increase in a $\mathrm{CD} 8 \alpha^{+} \beta^{\text {low }}$ subset that is concurrent with a reduction in CD8 $\alpha^{+} \beta^{\text {high }}$ of CD8 T-cells and involves lymphocyte populations from peripheral blood, lymph nodes, and thymus. ${ }^{212,334,374,376-379}$ A similar expansion of an $\mathrm{CD} 8 \alpha^{+} \beta^{\text {low }}$ T-cell subset has also been observed in HIV-1 infection. ${ }^{380,381}$ Furthermore, this cell population has been shown to express markers associated with lymphocyte activation and adhesion and to exhibit antiviral activity ${ }^{31,376,379}$ comparable to a noncytolytic CD8 antiviral activity previously described for HIV-1 infection. ${ }^{382,383}$

FIV-induced immunodeficiency is characterized by other defects that are similar to those described for HIV-1 infection and include reduced proliferative T-cell responses to mitogens, dysregulation of cytokine networks, and humoral immune response deficits. ${ }^{14,17}$ Several studies have revealed significantly reduced mitogen-induced blastogenic responses from T-cells isolated from cats during acute and chronic FIV infection,,$^{8,309,384-387}$ a finding that may result from virus-associated defects in T-cell growth and proliferation, as well as defects in cell surface expression of receptors required for transmission of antigen/mitogen signals. ${ }^{388}$ Although cytokine responses in different lymphoid tissues harvested from cats acutely infected with FIV have been shown to be heterogenous, ${ }^{389}$ findings from a number of reports suggest that dysregulation of cytokines such as IL-10, tumor necrosis factor (TNF)-alpha, IL-6, IL-1, and interferon gamma may play a role in FIV-induced immune deficiency. ${ }^{307,389-395}$ Deficits in generation of antibody responses to multiple antigens and specifically to T-celldependent immunogens have also been reported for FIV-infected cats. ${ }^{14,396-398}$ Bone marrow abnormalities that may result in neutropenia, leukopenias, and pancytopenias, ${ }^{323,327,363,399}$ as well as deficits in neutrophil and monocyte/macrophage function, may further impair immunologic function in FIV-infected cats. ${ }^{203,400-402}$

Histologic lesions associated with FIV infection are predominantly localized to lymphoid tissues including GALT for both early and later stages of FIV infection, although severity of acute stage lesions is dependent on virulence and titer of the infecting FIV isolate. Lymph node abnormalities during the acute stage of infection include a mixture of follicular hyperplasia and lymphoid depletion resulting in loss of lymph node architecture and medullary plasmacytosis., ${ }^{3,712,22,190,200,322,353,403}$ Lymphoid hyperplasia has also been observed in other lymphoid tissues including spleen, bone marrow, and mucosal-associated lymphoid tissue. Several studies have shown the thymus to be a primary target during early FIV infection, with pathologic changes that include thymic atrophy, thymitis, medullary B-cell hyperplasia, and cortical involution.,22,185,190,193,200,328,374 Lesions in the gastrointestinal tract have frequently been observed and include severe inflam- 
mation, necrosis, and villous atrophy., ${ }^{3,22,200,322}$ Brain and lung are nonlymphoid tissues that also frequently exhibit pathologic changes. CNS lesions have consistently included gliosis, glial nodules, and perivascular cellular infiltrates and less frequently included inflammatory changes associated with encephalitis and meningitis and neuronal abnormalities such as neuronal stress and neuronal satellitosis. ${ }^{198,200,324,329,330,332,339,342,344}$ Giant cell formation is a frequent lesion in SIV and HIV-1-associated neuropathology but has only rarely been observed by histologic examination of CNS tissues from FIV-infected cats. ${ }^{342,404}$ Pulmonary lesions associated with FIV infection have consisted of inflammatory infiltrates suggestive of pneumonia. $200,322,332$

Lymphoid depletion and involution within multiple lymphoid tissues have been dominant and consistent findings in tissues harvested from cats during the later stages of FIV infection., ${ }^{3,322}$ Neoplastic lesions, including B-cell lymphomas and other sarcomas, have also been frequently observed in later stages of both experimental and natural FIV infection. ${ }^{8,13,405-409}$ All together, these histologic lesions of lymphoid hyperplasia frequently characterized as B-cell hyperplasia mixed with severe lymphoid depletion, as well as thymic atrophy and the severe inflammatory lesions observed in the gastrointestinal mucosa, mimic pathological changes reported for both HIV-1 and pathogenic SIV ${ }^{364,410-413}$ and further support the use of the FIV animal for examination of HIV immunopathogenesis.

Mechanisms for either FIV or HIV-1-induced CD4 T-cell depletion and dysfunction in vivo most likely involve multiple processes such as direct cytolysis from virus infection or from virus-specific immune responses, and indirect strategies including chronic immune activation, cellular dysregulation, and inappropriate killing of uninfected bystander cells. ${ }^{364,414}$ In vitro syncytia formation of FIV-infected cells has been well documented and is due to viral envelope fusion with cellular membranes and associated with specific cell types and FIV strains. ${ }^{24,238,245,248,311,415-417}$ Programmed cell death or apoptosis has been hypothesized as one of several important mechanisms involved in FIV/HIV-1 immunopathogenesis based on observations of apoptotic cells in virus-infected cell cultures ${ }^{418-421}$ and in PBMC $^{422-425}$ and lymphoid and thymic tissues ${ }^{22,425,426}$ isolated from FIV-infected cats. Recent reports have shown that binding of cell surface chemokine and virus receptor CXCR4 by FIV TM may trigger apoptosis. ${ }^{137,427}$ Although some recent reports suggest that the Fas-TNF- $\alpha$ receptor pathway may be important for FIV-induced apoptosis, mechanisms by which FIV uses this pathway for apoptosis are not well understood and are currently under investigation. ${ }^{419,425,428-431}$

A second probable pathway for FIV-induced apoptosis involves the B7CTLA4 pathway. Data from recent reports indicate that PBMC and lymph node lymphocytes isolated from FIV-infected cell cultures in vitro or freshly isolated from FIV-infected cats are down-regulated for expression of 
costimulatory molecule CD28. These cells are instead up-regulated for expression of T-cell costimulatory molecule CTLA4, a cell surface molecule expressed on activated T-cells and reported to induce anergy in activated T-cell subsets. ${ }^{432-435}$ Furthermore, these reports presented flow cytometric analyses that verify induction of apoptosis in FIV-infected cultures by T-cells that coexpress CTLA4 and B7 cell surface molecules and suggested that CD4 T-cell depletion and lymph node apoptosis in vivo may partially result from chronic B7-CTLA4-mediated T-cell interactions. ${ }^{357,432,434}$ Similarly, reduced CD28 expression and increased CTLA-4 expression have been observed in HIV-1 infection ${ }^{436-440}$ and hypothesized to promote immune hyporesponsiveness and apoptosis through binding of CTLA4 with B7 costimulatory molecules. Although mechanisms by which FIV infection induces an increase in B7+CTLA4+ T-cell populations in vivo are not well understood, chronic immune activation in the virus-infected host has been hypothesized as one possible etiology for this effect. These observations further illustrate similarities in FIV and HIV-1-associated disease and potential value of the FIV animal model for in vivo studies focused on mechanisms of immunopathogenesis of lentivirus-induced acquired immunodeficiency.

\section{Immune Responses}

Emergence of cellular and humoral host immune responses during primary infection with either FIV or HIV-1 coincides with reduction in peak peripheral blood virus loads. ${ }^{14,17,24}$ These responses are thought to be important for controlling virus replication during acute and chronic stages of infection based on CD8 T-cell and B-cell depletion studies involving SIVinfected rhesus macaques. ${ }^{44-443}$ Regardless of robust virus-specific immune responses, most infected hosts fail to eliminate the virus, leading to prolonged clinical latency, eventual immunologic exhaustion with subsequent increasing virus loads, and AIDS. Currently, factors responsible for this inability of host responses to effectively clear virus during primary infection are not well understood. Identification of such factors will be necessary for understanding immunopathogenesis of lentiviral infections and design of vaccines capable of inducing sterilizing immunity.

Based on experimental inoculation studies, antibodies to viral proteins SU gp95, CA p24, and MA p14 are the first to appear in serum, usually within 2 to 4 weeks after infection, and are quickly followed by the appearance of antibodies to TM gp40, Gag precursor p50, and reverse transcriptase. ${ }^{14,28,444,445}$ Lentiviral Gag proteins are highly expressed immunogenic proteins and FIV-infected cats typically exhibit high titers of antibody specific for viral CA p24. Four B-cell epitopes have been mapped for FIV CA using mouse monoclonal antibodies. ${ }^{446}$ However, evidence that CA-specific antibodies function in either FIV or HIV-1 clearance has been scarce. Inter- 
estingly, a recent examination of serological responses in HIV-1-infected patients undergoing prolonged antiretroviral therapy with structured treatment interruptions (STIs) showed that kinetics of CA-specific antibody responses revealed clear differences in patients' immune functions. Patients exhibiting rapid and large increases in CA antibody responses also experienced significantly decreased viral set points. ${ }^{447}$ Antibodies specific to CA have not been shown to express significant antiviral activity, ${ }^{448}$ but may instead reflect enhanced virus-specific CD4 helper activity that is predictive of the capabilities of the host's antiviral immune responses. ${ }^{447}$ Similar careful analyses of FIV Gag antibody responses have not been reported but may be useful for characterizing the value of these responses as markers that aid in distinguishing protective from nonprotective vaccineinduced immune responses.

Only antibodies that bind to surface domains of the envelope are thought to exhibit virus neutralizing activity, ${ }^{449}$ and accordingly, identification of envelope B-cell epitopes is critical to the characterization of potentially protective host immune responses. Multiple reports have identified the V3 region of SU, carboxy terminal of SU, and a highly conserved PID located within the ectodomain of TM as major immunodominant domains of FIV envelope (Figure 3)..$^{14,133,252,254,450-454}$ Four or more linear B-cell epitopes have been mapped within the V3 domain of SU, ${ }^{254,453}$ and other SU epitopes are localized within V4 and V5 domains. ${ }^{452,455,456}$ B-cell epitopes within extracellular TM include the PID previously discussed, a second domain within the extracellular membrane-proximal domain of TM that is downstream of the PID, and a third domain within the intracytoplasmic region of $\mathrm{TM}^{452,453}$ In contrast, the tryptophan-rich motif within the extracellular domain of TM that functions in virus fusion and infectivity was not found to be immunogenic. ${ }^{457}$

Although the V3 immunodominant domain is the only FIV Env determinant consistently shown to induce antibodies capable of neutralizing virus in vitro, ${ }^{252-254}$ determinants within domains V4 and V5, including residues 481 and 551, were also shown to confer broad neutralization resistance (BNR) in primary isolates passaged in vivo (Figure 3). ${ }^{455,458-462}$ The remaining FIV envelope linear epitopes mapped by binding assays are most likely inaccessible for neutralization due to the complex oligomeric structure and extensive glycosylation of native FIV SU, a property shared with HIV-1 SU. ${ }^{449,454,463}$ Expression of both neutralizing epitopes and tropism determinants by the FIV hypervariable V3 domain is an important function also described for the V3 domain of HIV-1 surface glycoprotein. ${ }^{14,17,24,73}$ It is also significant that FIV SU linear epitopes, capable of inducing neutralizing antibodies, are located within hypervariable regions of envelope that may change in response to selective pressures in vivo. ${ }^{141}$

Importantly, an epitope encoded within the FIV extracellular membrane-proximal domain of TM (designated as the TM3 epitope) is 
similarly positioned to that of a HIV-1 epitope (2F5), which is recognized by an extremely potent broadly neutralizing HIV monoclonal antibody (Figure 3). ${ }^{463,464}$ Although feline antisera raised against this FIV TM epitope was not shown to exhibit virus neutralizing activity in vitro, ${ }^{454}$ a peptide vaccine based on this epitope was shown to be capable of inducing partial immunity to FIV challenge. ${ }^{465}$ These observations suggest that this extracellular membrane-proximal TM3 domain may encode a neutralizing epitope conserved across multiple lentiviruses and may support further investigation of this TM determinant in FIV vaccine design.

Neutralizing antibodies emerge in the host as peak peripheral blood virus loads decline during primary infection and are thought to contribute to control of virus load and replication in lentivirus infections including HIV-1, SIV, and FIV. ${ }^{466}$ Control of infection by neutralizing antibody is also based on CD8 T-cell and B-cell depletion studies of rhesus macaques infected with either SIV or chimeric SIV/HIV-1 (SHIV) isolates ${ }^{441,443,467}$ and on reports describing successful passive immunization of either neonatal kittens or neonatal macaques with hyperimmune serum. ${ }^{468,469}$ However, despite apparent successes observed recently with therapeutic administration of exogenous highly potent neutralizing antibodies in nonhuman primate animal models, ${ }^{470}$ antibodies induced in the host by virus infection have been consistently ineffective in virus clearance, particularly within lymphoid tissue reservoirs. Furthermore, FIV and HIV-1 assay systems using established cell lines, rather than primary lymphocytes for virus infectivity or highly passaged viruses compared to primary strains directly isolated from infected cats, generate significantly different data regarding neutralization activity for identical serum samples. ${ }^{454,471-473}$ Notably, previous studies have shown that both HIV-1 and FIV primary isolates are resistant to neutralization by autologous sera, especially when primary PBMC are used to assay infectivity. ${ }^{471,474}$ However, more recent findings regarding HIV-1 neutralizing antibody biology ${ }^{466}$ and use of more sophisticated approaches for analysis of antibody activity have triggered renewed interest and support for investigation of humoral immune responses in virus control during acute and chronic HIV-1 infection and for HIV-1 vaccine design. Similar investigations seem warranted in the FIV animal model, especially for examination of antibody kinetics and specificity associated with mucosal versus parenteral routes of virus exposure ${ }^{475}$ and for investigation of epitopes conserved across different virus clades as vaccine immunogens. ${ }^{476,477}$

Strong supporting data exist for CD8 T-cell-mediated suppression of virus load in both HIV-1-infected patients and SIV-infected rhesus macaques. $^{24,383,442,478,479}$ Investigation of FIV-specific cellular immune responses has previously been restricted by a deficiency of key felinespecific reagents, including antibodies specific for feline cell surface markers and cytokines, as well as a lack of knowledge of FIV T-cell epitopes. However, recent peptide mapping of FIV-specific T-cell epitopes ${ }^{480,481}$ and 
established assays for FIV-specific CD4, CD8, and cytotoxic T-cell (CTL) activity have now permitted some characterization of cellular immune responses induced by either virus infection or vaccination..$^{308,481-491}$ Furthermore, a report showing that adoptive transfer of blood cells isolated from FIV-vaccinated cats induced resistance in MHC-matched recipient cats to FIV challenge infection, provides some evidence of the importance of virus-specific cellular responses in virus clearance. ${ }^{488}$

Longitudinal examinations of cats experimentally infected with FIV reveal virus-specific CTL activity in PBMC within 2 to 7 weeks after infection, time points in primary infection that coincide with rising virus loads and are similar to those reported for CTL emergence in HIV-1 infection. ${ }^{24,475,482,490,492}$ Virus-specific CTL activity has also been detected in lymphocytes isolated from lymphoid tissues including systemic and peripheral lymph nodes and spleen during the primary phase of infection. ${ }^{475,482,492}$ Assay for CTL activity during chronic stages of infection at 47 and 127 weeks after virus exposure demonstrated persistence of cellular responses, although detection of antiviral CTLs were more consistent from lymphoid tissues compared to peripheral blood. ${ }^{482,492}$ Activity specific to FIV Gag rather than Env was more consistently observed in FIV-infected cats described in these reports. However, currently described protocols testing for FIV-specific CTL by chromium release assay are restricted by use of only two immunogens provided by vaccinia recombinant viruses, one of which expresses a Gag protein derived from isolate FIV-GL14 (subtype A) and the other expressing an Env protein encoded by FIVPetaluma (also subtype A). Therefore, lower Env-specific CTL activity may be partially attributed to deficient assay detection due to variability within recently mapped Env-encoded T-cell epitopes ${ }^{481}$ since cats described in these reports were experimentally infected with FIV isolates distinct from FIV-Petaluma. Measurement of CTL responses to variable proteins such as Env will require virus-specific reagents for optimal assay sensitivity. Characterization of FIV-specific CTL responses will also call for assay of activity against other viral proteins including RT, Rev, and Orf-A. Although limited mapping of Env T-cell epitopes was previously reported, ${ }^{493}$ a recent report $^{481}$ described peptide mapping of FIV T-cell epitopes across all FIV genes using a feline interferon gamma ELISpot assay. ${ }^{489}$ These newly identified epitopes will facilitate use of peptides for other FIV CD8 assays and further characterize FIV CD8 responses to different FIV isolates, as well as examine the occurrence of FIV CTL epitope escape variants, a well-described trend in SIV and HIV infection. ${ }^{494-496}$ Another unique test for FIV-specific CTL activity involved assay of perforin expression ${ }^{488,497}$ and may also be useful for future examination of FIV-specific CD8 immune responses.

Interestingly, a $\mathrm{CD} 8 \alpha^{+} \beta^{\text {low }}$ T-cell subset associated with antiviral activity in FIV-infected cats has actually been more carefully examined than the topic of virus-specific CTL activity. This cell population has also been 
described for HIV infection ${ }^{380,381}$ and was initially distinguished for its expansion in peripheral blood during the primary phase of FIV infection and for a capacity to suppress virus replication in cultured PBMC by release of a soluble factor in vitro. ${ }^{308,334,377,378,486,498}$ Significantly, appearance of this cell population correlated with reduction of peak PBMC-associated virus, suggesting possible antiviral activity in vivo as well as in vitro. ${ }^{308}$ Other FIV infection studies have confirmed an expansion of this $\mathrm{CD} 8 \alpha^{+} \beta^{\text {low }}$ T-cell subset $^{212,374}$ and also reveal expression of lymphocyte activation and adhesion markers by this subset. ${ }^{376,499}$ However, these studies also show conflicting data on the issue of a restriction of antiviral activity to the $\operatorname{CD} 8 \alpha^{+} \beta^{\text {low }}$ subset with some data suggesting that both $\operatorname{CD} 8 \alpha^{+} \beta^{\text {low }}$ and $\operatorname{CD} 8 \alpha^{+} \beta^{-}$subsets are capable of noncytolytic antiviral activity. ${ }^{311,379,492,499-502}$ A lack of agreement is also apparent among studies regarding correlations between CD8 T-cell noncytolytic antiviral activity and either CD4 T-cell counts, clinical disease in vivo, distribution patterns of this cell subset in blood and lymphoid tissues, or persistent expansion of the $\mathrm{CD} 8 \alpha^{+} \beta^{\text {low }}$ subset throughout the course of virus infection. ${ }^{212,308,376,379,503}$ More recent reports indicate that CD8 antiviral activity production could be enhanced or induced in vitro by exposure to either virus-infected cells or to cells expressing an irrelevant antigen, but is not induced by mitogen activation. ${ }^{334,502,504}$ These conflicting reports indicate that additional studies using standardized methodologies will be needed for a more precise definition of this CD8 T-cell antiviral activity. However, data generated from almost all studies reported so far suggest that this noncytolytic CD8 antiviral activity is reminiscent of a still undefined secreted CD8 antiviral factor (CAF) previously described for HIV-1 infection. ${ }^{382,383}$ Noncytolytic CD8 antiviral factors associated with both HIV-1 and FIV infections, although not clearly defined, have been hypothesized to be associated with the innate immune system. Multiple factors proposed in previous reports to represent human CAF include human $\beta$-chemokines RANTES, MIP-1 $\alpha$, MIP-1 $\beta$, MCP-1, SDF-1, and alpha defensins. ${ }^{478}$ These proteins, however, do not meet the criteria of CAF definition, ${ }^{478}$ either due to their biochemical nature or because they are not exclusively expressed by CD8 T-cells. Observation of CD8 T-cell antiviral activity in both HIV-1 and FIV infection confirms the importance of this host immune response to lentivirus infection and provides another opportunity for use of the FIV animal model for assessment of HIV-1 immunopathogenesis.

\section{FIV VACCINE DEVELOPMENT}

A safe efficacious vaccine that prevents the spread of HIV will be essential to arresting the spread of the AIDS epidemic. Studies with nonhuman primates and SIV and chimeric SHIV isolates have demonstrated that 
live-attenuated viruses are highly effective $;^{505-507}$ however, such vaccines maintain a low level of pathogenicity. ${ }^{508-510}$ Other vaccine trials in the rhesus macaque animal model have described noninfectious DNA vaccines that control viremia and suppress clinical disease but do not induce sterilizing immunity against SIV/SHIV infection. ${ }^{511-513}$ FIV vaccine research and development have been fairly well supported due to the value of this animal model for HIV vaccine development and to the significance of FIV as a natural pathogen in cats. A wide variety of vaccine approaches have been examined, although efforts have concentrated on particular vaccine methods including whole killed virus (WKV)-based vaccines, DNA vaccines, and viral protein subunit vaccines. ${ }^{514} \mathrm{~A}$ commercial FIV vaccine (Fel-O-Vax FIV, Fort Dodge Animal Health), containing whole killed viruses representing two distinct FIV subtypes, was approved by the USDA in 2002 for use in the domestic pet cat population and is one of only two commercial lentiviral vaccines currently in use, including a live attenuated EIAV vaccine widely used in China. ${ }^{515,516}$ However, use of this FIV WKV vaccine is still not widely accepted due to significant issues including interference of vaccine-induced antiviral antibodies with commercial FIV diagnostic assays $^{517}$ and the potential of vaccine-induced enhancement of virus infection. ${ }^{485,518-520}$

\section{FIV WKV and Fixed-Cell Vaccines}

FIV immunization studies based on conventional WKV or fixed virusinfected cell (FG) vaccines have produced a diverse array of experimental findings. Vaccine efficacy for different and identical FIV WKV-based vaccines has varied greatly, most likely due to modifications of vaccine inactivation procedure, producer cell types used, vaccination schedule, vaccine adjuvants, vaccine doses, routes of challenge, and variability in virulence or subtypes of challenge FIV isolates. ${ }^{514}$ WKV-based vaccine studies have reported both complete or partial protection against challenge with homologous and sometimes heterologous FIV isolates and have also described vaccine-induced enhancement of FIV infection. Interestingly, enhancement of challenge virus infection has been more frequently associated with FC vaccines prepared with autologous feline lymphocytes. ${ }^{485,518,519}$ In contrast, FC and WKV vaccines based on virus-infected cell lines have proven efficacious, although protection may be considerably reduced against challenge with either heterologous or more virulent FIV isolates. ${ }^{487,521-526}$ The current commercial FIV WKV vaccine is composed of two isolates including FIV-Petaluma subtype A and FIV-Shizuoka subtype D, specifically to broaden virus-specific immune responses, and has demonstrated improved protection against multiple subtypes when compared to single subtype WKV vaccines. ${ }^{487,527}$ Use of a FIV WKV vaccine as a booster to a priming 
immunization with a recombinant canarypoxvirus (ALVAC)-based FIV vaccine also improved protective responses against challenge with isolates distinct from the vaccinating strains. ${ }^{528}$ Duration of WKV and FC vaccineinduced protection has been another concern, with some vaccine studies revealing a breakthrough in vaccine-induced protection a year after vaccination despite boostering the primary immunization. ${ }^{529,530}$ Another concern relates to differences in WKV or FC vaccine-induced protection observed against similar challenge viruses but delivered by different routes of exposure, including parenteral and mucosal delivery. ${ }^{56}$ Collectively these findings suggest that WKV vaccines demonstrate significant potential for development of lentivirus vaccines, but that multiple issues including WKV vaccine-induced enhancement still require attention for achievement of optimal protection.

Conclusions regarding neutralizing antibodies as immune correlates of vaccine protection frequently varied in early reports describing WKV and FC FIV vaccines. ${ }^{525,531-533}$ Nonetheless, other early studies, as well as more recent reports, present findings that suggest a correlation between appearance of virus neutralizing antibodies and $\mathrm{WKV}$ and $\mathrm{FC}$ vaccine-induced protection. ${ }^{487,522,523,526,534,535}$ WKV and FC vaccines have also induced potent cellular immune responses that are thought to be important correlates of protection. ${ }^{484,487,497,535-537}$ The current commercial FIV WKV vaccine containing inactivated whole viruses of subtypes $\mathrm{A}$ and $\mathrm{D}$, elicited strong cellular responses against both vaccine strain viruses and moderate neutralizing antibody activity, particularly when commercial Fort Dodge vaccine adjuvant FD-1 was supplemented with human IL-12. In fact, the commercial dual subtype FIV WKV vaccine administered with human IL12 provided broad protection against homologous and heterologous virus challenges containing in vivo-derived FIV inoculum. ${ }^{487,497}$ However, the actual contribution provided by cellular and humoral immune responses to protection induced by this vaccine approach is still not clear and may ultimately require immune cell depletion studies for determination. Despite reports of FIV WKV and FC vaccine efficacy, acceptance of this vaccine approach in the veterinary community is restricted by concern of vaccine antibody interference with FIV antibody-based diagnostics. ${ }^{517}$ Furthermore, exploration of WKV vaccines in the SIV and SHIV animal model has been extremely limited and was discouraged by early studies showing SIV WKV vaccine-induced protection resulted from immune responses to cellular antigens in vaccine preparations, rather than to viral antigens. ${ }^{538,539}$ However, renewed enthusiasm for vaccines that induce strong neutralizing antibody responses ${ }^{466}$ has lent increased support for multiple modality vaccine approaches that include WKV as a component. Inclusion of a WKV vaccine as a component is expected to enhance and broaden virus-specific humoral immune responses induced by other vaccine components such as 
live viral vectors and DNA. ${ }^{540,541}$ The sizable body of data already generated for FIV WKV and FC FIV lends strong support for use of the feline animal model for further investigation of $\mathrm{WKV}$ and $\mathrm{FC}$ vaccine approaches for HIV-1.

\section{FIV Subunit and Peptide Vaccines}

Various FIV subunit vaccine approaches have been tested including SU (primarily V3 and C2 epitopes), TM and Gag peptides, ${ }^{465,480,483,493,524}$ recombinant $\mathrm{SU}$ proteins generated from bacterial expression plasmids administered as single immunogens, ${ }^{542,543}$ or in combination with recombinant SU proteins expressed from vaccinia ${ }^{462,472}$ or baculovirus vector systems, ${ }^{544,545}$ and immunoaffinity-purified SU from FIV-infected cell lysates. ${ }^{531}$ Absence of protection against FIV challenge, observed with many of these frequently highly immunogenic subunit approaches, recapitulates findings reported for subunit vaccine approaches in the nonhuman primate model ${ }^{546}$ However, partial protection against challenge infection was revealed in a few reports describing immunization with either immunoaffinity-purified $\mathrm{SU}^{531}$ or SU proteins conjugated to autologous erythrocytes via biotin-avidin-biotin bridges,${ }^{547}$ although immune correlates of protection were not determined. As described in the section on immune responses, a FIV peptide vaccine based on an extracellular TM3 epitope, similarly positioned to that of the highly neutralizing HIV-1 2F5 epitope, was also shown to be capable of inducing partial immunity to FIV challenge. ${ }^{455}$ Vaccine-induced enhancement of FIV challenge virus infection has also been observed, with a subunit approach consisting of coimmunization of a bacterially expressed full-length envelope protein with either SU or SU-TM vaccinia recombinant proteins. ${ }^{462}$ However, coinoculation of recombinant $\mathrm{SU}$ proteins, with a plasmid expression vector encoding the FIV NC protein, abrogated vaccine-induced enhancement of challenge infection that was observed for vaccination with recombinant SU alone ${ }^{543}$ and actually improved vaccine efficacy in another study. ${ }^{545}$ Loss of vaccineinduced enhancement and increased vaccine efficacy associated with coinoculation of NC expression plasmid with recombinant SU were hypothesized to result from a plasmid DNA adjuvant effect based on altered cytokine expression profiles and the absence of FIV NC-specific immune responses associated with this approach. All together, these data do not support use of vaccines consisting of single viral protein subunits. Instead, these findings suggest that FIV may provide another vaccine model for testing peptides encoding broadly reactive neutralizing epitopes as components of a multiple modality vaccine that also includes immunogens for induction of cellular responses, a balanced vaccine approach currently of interest in HIV-1 vaccine design. ${ }^{466,548}$ 


\section{FIV DNA Vaccines}

Assessment of other vaccine approaches, including DNA vaccines, attenuated virus vaccines, and live attenuated viral or bacterial vectors for expression of FIV antigens, is still somewhat limited at this time. FIV DNA vaccine approaches, based on deletion mutants of FIV provirus plasmids, have shown considerable efficacy when challenged with less virulent FIV isolates. ${ }^{174,314,331,549}$ DNA vaccines, including either defective proviruses or SU and TM expression cassettes along with feline cytokine expression vectors, have shown particular promise in limited studies. ${ }^{314,549-551}$ Extremely low or absent humoral immune responses observed with FIV DNA vaccines may partially result from failure to incorporate codon optimization of viral structural genes encoded by these vaccine plasmids, a process found critical for improved immunogenicity of HIV-1 DNA vaccines that do not coexpress the viral Rev protein. ${ }^{552}$ DNA vaccine efficacy has been observed despite low antibody responses and may be attributed in some measure to strong virus-specific CTL responses, ${ }^{553}$ despite the lack of a clear correlation between measured CTL activity and DNA vaccine-induced protection. ${ }^{314}$ Unfortunately, DNA vaccine-induced protection has proven to be reduced or negligible against more pathogenic isolates. ${ }^{314,523}$ Moreover, DNA vaccines encoding envelope genes used without cytokine adjuvants have been associated with enhancement of challenge virus infection..$^{554-556}$

\section{FIV Vaccines Using Live Viral and Bacterial Vectors}

Mixed results regarding efficacy have been reported for the few FIV vaccines utilizing either live viral or bacterial vectors. Immunization of cats with a replication-defective adenovirus type 5 expressing FIV Env was poorly immunogenic and failed to induce protection against FIV challenge. ${ }^{557} \mathrm{~A}$ vaccine composed of Venezuelan equine encephalitis (VEE) virus replicon particles engineered to express the FIV matrix/capsid region of Gag and full length Env also failed to induce protection against FIV challenge, although the vaccine elicited both humoral and cellular responses. ${ }^{558}$ A combination vaccine using priming inoculations with a feline herpes virus (FHV) vector expressing the FIV gag gene and a FHV vector expressing FIV env and booster inoculations with a FIV FC vaccine also failed to protect immunized cats from FIV challenge infection. ${ }^{521}$ In contrast, partial protection against FIV challenge was observed for cats immunized with live attenuated Salmonella typhimuriaum aroA strains expressing FIV CA and truncated SU encoding hypervariable regions V3-V5, although correlates of protection were not defined. ${ }^{559}$ Partial protection against challenge was also observed by vaccination of cats with a recombinant canarypoxvirus (ALVAC)-based FIV vaccine used alone or in combination with a FIV FC vaccine ${ }^{528}$ Furthermore, a single oral immu- 
nization of cats with a live recombinant Listeria monocytogenes strain, which both expresses FIV Gag and delivers an FIV truncated Env-expressing plasmid, was found to confer protection by reduction of virus load and virus-induced disease after FIV challenge. ${ }^{560}$ The limited number of FIV vaccine studies utilizing live viral vectors is surprising, given the interest and success of multiple modality vaccines consisting of live recombinant viral vectors and DNA expression plasmids recently observed for the SIV/SHIV animal models. ${ }^{513}$

\section{FIV Attenuated Virus Vaccines}

Attenuated live virus vaccines have been extensively examined in the SIV/SHIV animal models due to consistent efficacy shown by these viruses for inducing protection against challenge with highly pathogenic SIV and SHIV isolates. ${ }^{505-507,561}$ A widely used vaccine based on an attenuated strain of equine lentivirus EIAV has protected 75 million horses and donkeys over the past 30 years in China. ${ }^{515,516}$ Studies describing efficacious molecularly cloned attenuated EIAV and CAEV vaccine viruses have also been reported. ${ }^{562,563}$ Examination of attenuated FIV vaccines has been limited but shown that this approach is also effective for the feline AIDS animal model. The FIV-Petaluma virus variant used in the commercial FIV WKV vaccine was recently shown to establish a low-level infection of cats without induction of apparent FIV-associated disease. ${ }^{316}$ Furthermore, infection with the attenuated FIV-Petaluma variant protected against infection with a different pathogenic FIV isolate (FIV GL8), although of the same subtype. Complete protection was conferred by this attenuated FIV against FIV GL8 delivered by the intraperitoneal route and partial protection was demonstrated against challenge by a mucosal route. Complete protection was also imparted against wild-type FIV challenge by inoculation of cats with infectious molecularly cloned FIV deletion mutants including a LTR mutant encoding a deletion of the AP-1 site $^{564}$ and a vif-deleted provirus (FIV- $\Delta$ vif) administered as a DNA vaccine. ${ }^{174,331}$ Lastly, superinfection of domestic cats with either nonpathogenic lion or puma nondomestic feline lentiviruses has been shown to confer resistance to infection with pathogenic domestic FIV. ${ }^{565}$ Immune correlates were not apparent from any of these attenuated FIV vaccine studies. In fact, the substantial genetic variation observed between domestic and nondomestic feline lentiviruses argues against initial control of challenge virus infection driven by epitope-specific immune responses and instead suggests currently undetermined resistance factors or mechanisms possibly associated the innate immune system. An important limitation of attenuated lentiviral vaccines is the concern for longterm safety as demonstrated by experiments performed by Ruprecht and coworkers, which showed that a SIV nef deletion mutant may cause fatal AIDS-like disease in newborn macaques and may become pathogenic after 
long-term infection of adult macaques. ${ }^{508-510}$ Regardless of whether attenuated lentivirus vaccines will ever be safe enough for general use, examination of these viruses in animal model systems, including FIV, offers an important opportunity to identify and fully characterize immune responses and undefined host resistance factors that confer protection against pathogenic virus infection or disease.

\section{Critical Issues for FIV Vaccine Development}

FIV vaccine development faces critical issues that are very similar or identical to those facing HIV-1 vaccines. Characterization of immune correlates of protective immunity for any of the FIV vaccine approaches examined so far has been elusive, a situation that is common to all lentivirus vaccines including those for SIV and SHIV, and must be addressed for successful HIV-1 vaccine design in the future. ${ }^{566}$ Vaccine-induced enhancement of pathogenic virus challenge has been consistently observed with FIV and EIAV vaccine approaches, ${ }^{567}$ involving either WKV, FC, or protein subunits/peptides and for FIV, was thought to result from enhancing antibodies that might be specific to epitopes within Env hypervariable regions V3-V5 or to the PID epitope. ${ }^{462,568,569}$ However, vaccines using recombinant Env proteins with regions V3-V5 deleted were shown to remain capable of inducing enhancement of challenge infection. ${ }^{569}$ Occurrence of FIV DNA vaccine-induced enhancement of challenge virus infection despite negligible antibody responses, also suggested mechanisms other than enhancing antibodies, such as lymphoid activation. ${ }^{554,555}$ A very recent report described enhancement of challenge virus infection after vaccination of rhesus macaques with an attenuated recombinant varicella-zoster virus vaccine expressing SIV Env. ${ }^{570}$ Vaccine-associated enhancement in this study correlated with appearance of robust anamnestic virus-specific CD4 proliferative responses in the absence of strong CD8 responses and again suggested that immune activation may play a role in vaccine-induced enhancement along with cellular responses skewed against CD8 T-cells. These findings suggest major concerns for clinical testing of HIV-1 vaccines for which less than optimal CD8 responses are already predicted and validate use of the FIV animal model for characterization of this potentially devastating complication of HIV vaccine use. A third issue of importance for both future and the current commercial FIV vaccine design as well as future commercial HIV-1 vaccines, concerns interference of vaccineinduced antibodies with FIV diagnostics. All lentivirus diagnostic assay systems utilize seropositivity as a marker for virus infection. PBMC virus isolation and plasma viral RNA detection systems using PCR, although specific, are not sufficiently sensitive to detect all virus infections or currently feasible as routine diagnostic assays. Future HIV-1 vaccine design will even- 
tually need to address this issue for which the FIV animal model may prove most useful.

\section{FIV AND ANTIVIRAL THERAPIES}

Relatively soon after the initial isolation and characterization of FIV, considerable efforts were focused on characterizing this lentivirus as an animal model for antiviral therapies for HIV-1. These studies were encouraged by similarities between FIV and HIV-1 RT in amino acid sequence, structure and physical properties, catalytic activities, and susceptibility to multiple nucleoside analogs, including AZT, 3TC, 9-(2phosphonomethoxyethyl)adenine (PMEA) and (R)-9-(2-phosphonylmethoxypropyl)-2,6-diaminopurine $\{(\mathrm{R})$-PMPDAP $)\},,^{571-575}$ as discussed earlier in this chapter. Conversely, FIV has proven relatively resistant to therapies including nonnucleoside RT inhibitors and protease inhibitors used in highly active antiretroviral therapy (HAART) protocols for HIV-1infected patients. ${ }^{96,109,110,576}$ Nevertheless, ongoing studies are evaluating protease inhibitors in vivo that show activity against proteases of multiple lentiviruses in vitro, as well as drugs that target lentiviral TM fusion domains conserved across different lentiviruses. Identification of such compounds will be important for design of efficacious HAART protocols for FIV infection and continued development of FIV as a model for HIV-1 antiviral drug therapy.

Examination of FIV susceptibility to AZT in vitro resulted in the first description of emergence of an AZT-resistant lentivirus through virus passage in cell culture ${ }^{104}$ and led to a rigorous examination of multiple drug-resistant FIV RT mutants that arise in vitro. ${ }^{108,577-579}$ Although FIV resistance to AZT did not map to homologous residues within AZT-resistant HIV-1 RT mutants, both FIV and HIV-1 do share a similar determinant (M184 in the YMDD active site of the RT palm subdomain) for susceptibility to nucleoside analog 3TC. ${ }^{106,107}$ Accordingly, a Met-to-Thr mutation in the YMDD motif of RT has been observed in 3TC-resistant mutants for both FIV and HIV-1. Studies assessing drug-resistant FIV mutants in vivo have been very few but have revealed attenuation of an AZT-resistant FIV when inoculated into cats. ${ }^{111}$ These findings recommended the potential use of this model for characterizing pathogenesis of other FIV variants resistant to drugs, targeting domains conserved between lentiviral enzymatic proteins.

Examination of FIV susceptibility to nucleoside analogs AZT, PMEA, and (R)-PMPDAP in vitro has been complemented by a variety of in vivo studies that illustrate the value of this animal model for HIV-1 antiviral drug development and assessment of drug efficacy. Studies testing the merit of 
AZT as monotherapy for either reducing virus load, improving CD4:CD8 T-cell ratios, or clinical status in both experimental primary infection and chronic natural infection with FIV have produced somewhat mixed results. Altogether, however, investigations have revealed fairly limited efficacy for AZT used as a single therapeutic regimen for FIV infection, as previously found in HIV-1 infection, and frequently reported side effects such as anemia that are also observed in human patients. ${ }^{580-587}$ Furthermore, a single report examining combination therapy of AZT and 3TC for cats either acutely or chronically infected with FIV described only slightly improved efficacy compared to AZT treatment alone. ${ }^{588}$ In contrast, nucleoside analog PMEA and its derivative (R)-PMPDAP have proven very efficacious as antiviral therapies for FIV infection both in vitro and in vivo by significantly reducing virus load and improving CD4:CD8 T-cell ratios. ${ }^{572,573,575,581,589,590}$ Combination therapy consisting of AZT, 3TC, and a third nucleoside analog abacavir also blocked FIV replication in a synergistic manner in vitro. ${ }^{591}$ These observations suggest that this drug combination or combinations including PMEA may warrant future assessment as HAART protocols using the FIV animal model.

A shortage of HIV-1 protease inhibitors that effect FIV replication due to differences in substrate specificity displayed by FIV PR and HIV-1 PR ${ }^{90-95}$ has hampered use of the FIV animal for analysis of combination drug protocols used in HAART protocols for HIV-1-infected patients. However, attempts to identify a protease inhibitor universally active against multiple lentivirus PRs revealed that a statine-based inhibitor LP-130 $0^{96}$ and a C2symmetric competitive inhibitor identified as TL- $3^{93,592}$ were both capable of inhibiting PR expressed by HIV-1, SIV, and FIV. Interestingly, comparison of crystal structures of FIV PR and HIV-1 PR in complex with TL-3 reveals differences in the position of the flaps in FIV PR and HIV-1 PR, whereas complexes of FIV PR and HIV-1 PR with inhibitor LP-130 are nearly identical in conformation. ${ }^{593}$ Recent reports have shown TL-3 to inhibit FIV and HIV-1 replication and to be active against protease inhibitor-resistant HIV-1 mutants in vitro. ${ }^{93,592}$ Furthermore, TL-3 treatment of FIV-infected cats reduced virus load and disease, including clinical neurologic dysfunction and severe acute phase immunodeficiency. ${ }^{343,594}$ These findings support further testing of TL-3 in combination drug protocols for FIV infection both in cell culture systems and in vivo. Assessment of combination protocols with TL-3 may be particularly warranted for testing a proposed hypothesis that therapy with compounds broadly reactive against proteases of multiple lentiviruses will less likely be associated with emergence of protease inhibitor-resistant virus mutants in vivo.

Recent preliminary studies have also described testing synthetic peptides targeted to determinants within the FIV TM ectodomain, such as the heptad repeat 2 (HR2) domain ${ }^{595}$ and the membrane-proximal tryptophanrich region ${ }^{138,416}$ (Figure 3) as antiviral therapeutics. Previous studies 
showed that HIV-1 peptides derived from the HR1 and HR2 regions are potent inhibitors of HIV-1 infection and function by blocking virusmediated cell fusion. ${ }^{596}$ Similarly, the membrane-proximal tryptophan-rich region immediately downstream of HR2 within the TM ectodomain has also been shown to be important for virus-induced fusion and infectivity for both HIV-1 and FIV. ${ }^{34,137,597}$ Testing of combination FIV therapies that include nucleoside analogs, protease inhibitors such as TL3, and Env peptide inhibitors now proven active against FIV has not yet been reported but looks promising for further development of the FIV model for HAART therapies for HIV-1.

Various other antiviral approaches have shown activity against FIV replication either in vitro or in vivo but have been examined in a limited fashion. Strategies previously shown to successfully target the FIV Rev regulatory system in vitro include a ribozyme directed to the FIV RRE element ${ }^{598}$ and small molecule intervention using 1,8-diaminooctane that blocks the formation of hypusine required by eIF-5A, a cellular factor required for HIV-1 Rev function. ${ }^{166}$ Cytokines and cellular growth factors including recombinant human interferon-alpha2, human interferonomega, human interferon-tau, recombinant human GM-CSF, IL-16, and recombinant human insulin growth factor-1, demonstrated limited success as treatment for FIV infection either in cell culture systems or in infected cats. ${ }^{32,394,599-603}$ However, cytokines as adjunctive therapy to HAART therapy are currently under evaluation in HIV-1-infected patients ${ }^{604}$ and may be worthy of similar examination in the FIV model, where mechanisms may be experimentally characterized. Cytokines may be useful both as immune reconstitution therapeutics and for activating expression of latent reservoir viruses for subsequent elimination by antiviral drugs. In conclusion, animal models such as FIV and SIV provide opportunities for examination of tissues and cell subset reservoirs for virus in hosts undergoing HAART therapy as reported for HIV-1-infected patients ${ }^{232,605}$ and, more importantly, characterization of viral mechanisms for persistence within these reservoirs.

\section{FIV AS A VIRAL VEGTOR}

Advances in characterization of FIV molecular virology have facilitated development of FIV vectors as vehicles for gene transfer in both dividing and nondividing cells. ${ }^{606,607}$ FIV vector development has equally contributed significantly to the body of knowledge regarding viral determinants of FIV gene expression, virus packaging, and integration. Lentiviruses provide unique vector systems that allow reliable integration of foreign genes into chromosomal DNA of nondividing cells. Although cumulative data generated for HIV-based vectors suggest that safe and effective primate lentivirus 
vectors will be possible, concern over the clinical use of gene vectors derived from a pathogenic human retrovirus (HIV-1) may restrict the use of such vectors. FIV has provided the first nonprimate lentivirus vector system capable of gene transfer efficiency comparable to that observed with HIV-based vectors. ${ }^{608}$ Development of lentivirus vectors based on FIV has received continued support due to the apparent lack of FIV replication competence in human cells, resulting from restriction of FIV LTR promoter activity. Although cell culture-adapted variants of FIV are capable of utilizing human chemokine receptor CXCR-4 for infection of human cells, ${ }^{260}$ FIV LTR-directed gene expression and virus production are abrogated in human cells. ${ }^{415}$ Lack of cross-reactivity of FIV proteins with those of HIV is another potential advantage for use of FIV-based vectors. As discussed above, FIV virion structure and genomic organization are typical of other lentiviral genomes except for the absence of specific accessory and regulatory genes such as $v p r, v p u$, nef, and tat and the presence of a polencoded dUTPase. The less complicated FIV genome provides another advantage for use of this vector system. FIV vectors systems infect human primary cell types from a broad array of tissues, including brain, eye, airway, hematopoietic system, liver, muscle, and pancreas. ${ }^{607}$ These and other factors have promoted enthusiasm for FIV vector systems for gene therapy in human disease.

The first FIV vector described was derived from FIV molecular clone $34 \mathrm{TF} 10^{38}$ and documented that FIV-based vectors were capable of transducing nondividing human cells. ${ }^{260}$ Subsequent studies reported by other research groups described biology and cell tropism for similar but modified FIV vector systems also based on FIV 34TF10. ${ }^{25,609,610}$ All FIV vector systems described to date use three plasmids: a transfer vector for encoding the gene of interest, a packaging vector for expression of structural and enzymatic genes, and a plasmid for expression of the vesicular stomatitis virus (VSV)-G envelope protein for pseudotyping of vector particles. ${ }^{606} \mathrm{~A}$ chimeric FIV LTR, composed of a human cytomegalovirus (hCMV) immediate early gene promoter replacing the FIV U3 element and fused to the R/U5 LTR domains, is found in all FIV transfer vectors and is necessary for FIV vector production from human cells. As stated earlier, studies focused on optimization of FIV vectors system have mapped specific replication domains that include encapsidation determinants, ${ }^{44,45}$ a central polypurine tract, and a central termination sequence that generates a central DNA flap in the preintegration complex. ${ }^{46}$ Data from these studies revealed a divergence in sequence between the FIV cPTT and the FIV 3' PTT, and also showed that the FIV cPTT and $3^{\prime}$ PTT are not entirely purine. These findings uncovered differences between FIV and HIV-1 reverse transcription and have been incorporated into FIV vector design. Vector optimization studies have also characterized class I FIV integrase mutants that prevent the integrase reaction but do not perturb other Gag/Pol 
functions. ${ }^{125,607,611}$ These integrase mutants may be incorporated into preclinical gene therapy studies in animals for providing control vectors that identify tissues with a requirement for vector integration for gene expression. Finally, FIV vectors have been further modified by deleting noncoding and nonstructural FIV sequences as well as specific gag sequences for construction of minimal vectors with reduced cytotoxicity, yet efficient gene transfer. ${ }^{609,612}$ Continued FIV vector design studies will contribute to further understanding of the FIV life cycle and FIV molecular virology, as well as facilitate development of optimal lentiviral vector systems. Additional studies will also be required to fully characterize and compare relative efficiencies, as well as biosafety of FIV with primate lentivirus vectors.

\section{FELINE LENTIVIRUSES OF NONDOMESTIC CATS}

\section{Incidence}

The initial observation of infection of nondomestic cats with a feline lentivirus ${ }^{613}$ was subsequently confirmed by multiple reports revealing either virologic or serologic evidence of infection in both captive and freeranging populations of several nondomestic feline species. ${ }^{269,275-282}$ Speciesspecific feline lentivirus isolates have since been characterized for lions (FIV-Ple), leopards (FIV-Ppa), pumas/courgars (FIV-Pco), and pallas cats (FIV-Oma) ${ }^{277,614-617}$ Nondomestic feline lentivirus infection of free-ranging nondomestic felids is worldwide and includes lion and leopard populations within Africa; cougar populations in southern and western United States, Canada, and South America; cheetahs in Africa; and Pallas cats in central Asia. ${ }^{269,275,278,281,282,613}$ Interestingly, the prevalence of feline lentivirus infection in specific nondomestic cat populations, including lions in the Serengeti National Park and Ngorongoro Crater of east Africa and Kruger National Park of South Africa and cougars within the Snowy Mountain Rage in Wyoming, is quite high ranging from 58\% (cougars) to $90 \%$ (lions). ${ }^{277,281,282,618}$ Despite evidence of endemic infection of free-ranging lion and cougar populations, overt disease has not been associated with lentivirus infection in these populations. ${ }^{619,620}$ This apparent lack of virulence, along with the significant genetic diversity observed among feline lentiviruses, suggests that FIV-Pco and FIV-Ple are ancient viruses that have adapted to their hosts ${ }^{22,615,618}$ and may be comparable to African SIV isolates that are similarly nonpathogenic for their natural hosts. ${ }^{61,622}$

\section{Genomic Diversity}

Characterization of a molecularly cloned FIV-Oma proviral genome revealed genomic organization similar to that of domestic cat FIV iso- 
lates. ${ }^{614}$ However, current knowledge of genomic diversity of nondomestic FIV isolates is based primarily on phylogenetic analyses focused on viral sequences generated from endemic FIV-Ple- and FIV-Pco-infected cat populations. Although lentiviruses infecting nondomestic cats are clearly related to domestic cat FIV, comparison of amino acid and nucleotide sequences derived from the highly conserved pol gene of nondomestic (lion, cougar, and pallas cat) and domestic feline FIV isolates reveals considerable variation, ranging from 21 to $30 \%$ differences in nucleotides and 19 to $45 \%$ in amino acids. ${ }^{269,277,614,615,618}$ These data indicate that divergence between domestic and nondomestic cat lentiviral genomes is similar to that observed between HIV-1 and HIV-2. Furthermore, significant genomic diversity reported for cougar FIV-Pco isolates gathered from North and South America defined two principal clades and 15 divergent subclades for this nondomestic FIV. ${ }^{282,615}$ Genetic divergence measured for the two FIV-Pco clades was similar to the diversity that distinguishes domestic cat FIV from FIV-Ple isolates. Similarly, analyses of FIV-Ple isolates from lion populations within the Serengeti Reserve and Ngorongoro Crater in southeastern Africa identified three phylogenetic clades exhibiting genetic diversity also similar to that which separates domestic cat FIV from FIV-Pco. ${ }^{277,618}$ Sequence diversity within the conserved pol genes derived from both FIVPco and FIV-Ple isolates is greater than that reported for the few pol sequences available for domestic cat FIV. In fact, this pol gene variation is similar to diversity measured for the more variable $e n v$ genes used to define unique domestic cat FIV clades. These findings provide additional support to the theory that nondomestic feline lentiviruses are ancient viruses that have existed within cougar and lion species much longer than FIV in domestic cats.

Additional findings from phylogenetic analyses of endemic FIV-Pcoand FIV-Ple-infected cat populations include evidence of vertical transmission $(\mathrm{Pco})^{282,615}$ and possible coinfection of different subtypes within a single host (Pco and Ple) ${ }^{615,618}$ A recent report examining a large freeranging lion population that included 13 prides within the Serengeti National Park revealed the presence of all three FIV-Ple subtypes widely dispersed within the population. Infection with the three FIV-Ple subtypes within the same pride and possibly within the same host was also observed. ${ }^{618}$ Furthermore, a high incidence $(43 \%)$ of coinfection with two to three FIV-Ple subtypes within individual animals was reported, although the alternative possibility of infection with subtype-recombinant viruses was not ruled out. Significant genomic divergence between different FIV-Ple subtypes, most notable between subtype $\mathrm{C}$ and the two other subtypes (A and B), was also observed for this population of lions. Monophyletic clustering of FIV-Ple sequences was observed for only one of the prides under study. 
Findings described above for lion populations within Serengeti National Park contrasted with those generated from a study of a smaller population of wild cougars located in a mountain range in southeast Wyoming. ${ }^{282}$ These animals supported an overall FIV-Pco prevalence rate of $58 \%$ and a remarkable $100 \%$ rate of infection for all adult animals. Although analysis of both pol and env sequences confirmed the presence of two distinct FIV-Pco lineages within this infected cougar population, sequence diversity between the lineages was low and evidence of coinfection of a single host with viruses of distinct lineages was not found. Importantly, investigation of the evolution of both FIV-Pco pol and env sequences within this cougar population over time revealed mean rates of 1 to $3 \%$ per 10 years, a rate considerably less than rates of 0.3 to $1 \%$ per year reported for HIV. ${ }^{623-626}$ Collective observations generated so far from phylogenetic analyses of nondomestic FIV isolates suggest that these lentivirus infections provide unique opportunities to evaluate virus dynamics and viral sequence evolution in a natural host for a nonpathogenic lentivirus.

\section{Infection and Replication of Nondomestic Feline Lentiviruses}

The extent of genetic divergence described so far between domestic and nondomestic feline FIV isolates would suggest that infection and replication of nondomestic isolates may not be possible in domestic cats or in primary lymphocytes isolated from domestic cats. Reports of in vitro replication properties of nondomestic feline lentiviruses are scarce and have been restricted by the inability of some nondomestic FIV isolates such as cougar FIV-Pco, to replicate in domestic cat primary lymphocytes. ${ }^{269,617}$ However, some nondomestic FIV isolates including FIV-Ple and FIV-Oma have been shown to replicate in domestic cat lymphocytes and some feline cell lines including CrFK cells (FIV-Oma) and a feline lymphoid cell line (3201 cells) (FIV-Ple). ${ }^{275,617}$ FIV-Pco was also shown to replicate in 3201 cells. ${ }^{617}$ Although characterization of receptor usage for nondomestic feline lentiviruses has not yet been reported, these current findings for in vitro growth properties suggest that nondomestic FIV isolates may also be capable of infecting and replicating in vivo in domestic cats. Rare, isolated cases of naturally occurring cross-species transmission of domestic cat FIV to nondomestic cats have been reported, which involved a cougar and a wild-caught Tsushima cat belonging to a subspecies of leopard cats located in Japan. ${ }^{279,615}$ Experimental transmission studies have also confirmed the infectivity of both FIV-Pco and FIV-Ple in domestic cats ${ }^{627,628}$ and revealed that establishment of persistent FIV-Ple viral infection is possible in the absence of FIV-associated disease. Moreover, prior infection of domestic cats with either FIV-Pco or FIV-Ple appears to impart some resistance to challenge with pathogenic domestic cat FIV based on lower challenge virus 
loads and higher CD4 counts measured for FIV-Pco- and FIV-Ple-infected domestic cats compared to naive cats after exposure to domestic cat FIV. ${ }^{565}$ Additional studies with rigorous measurement of virus load and careful examination of viral immunopathogenesis over extended time periods will be needed to characterize nondomestic feline lentivirus infection of the domestic cat as a model for HIV infection.

\section{OUTLOOK FOR THE FIV ANIMAL MODEL}

Significant progress has been made in the development of antiviral drug regimens for HIV-1, although complete elimination of the virus from the host and full restoration of immunocompetency are still not possible. However, a commercial safe and efficacious vaccine for HIV-1 is still not available and is unlikely in the near future, despite exhaustive efforts in HIV-1 vaccine development that began 20 years ago immediately after the initial characterization of HIV-1. ${ }^{566}$ Several serious issues that must be resolved for future vaccine success include identification of immune correlates of protection against either lentivirus infection or virus-associated disease. The apparent difficulty of addressing this question was predictable since virus elimination has not been observed during natural infection with immunodeficiency-inducing lentiviruses, even when strong virus-specific cellular and humoral immune responses have been detected in the infected host. Vaccine-induced protection against pathogenic challenge has been difficult to achieve in both the SIV and FIV animal models, and when observed, has been inconsistent. An additional complication is presented by the specter of vaccine-induced immune responses that prove detrimental rather than protective as reported for FIV and more recently in the SIV animal model. The complication of virus strain diversity has barely been addressed by vaccine design in animal models and surely poses a major complication for HIV-1 vaccine success in the future. Successful resolution of these obstacles in vaccine development, and most particularly the lack of understanding of why some vaccines such as attenuated viruses are sometimes protective, absolutely requires continued examination of viral pathogenesis and host immune responses in animal models including both nonhuman primate and nonprimate lentivirus animal models. Elucidation of pathogenic mechanisms used by these immunodeficiencyinducing lentiviruses during the earliest stages of virus exposure and virus-host interactions will be critical for design of vaccines that will have any potential for sterilizing immunity, if such a goal is possible. Similarly, a far more precise understanding of viral mechanisms for immunodeficiency will also be necessary for design of therapies of immune reconstitution in both acutely and chronically HIV-1-infected hosts, which can only be gained through experimental animal model studies. 
Significant progress in characterization of FIV infection has been accomplished in the past 10 years, especially regarding molecular characterization of viral proteins, FIV receptor usage, and virus tropism and localization in vivo. Progress in development of FIV vectors for gene therapy has also been accomplished and has contributed considerably to the molecular characterization of FIV. Continued efforts in FIV vaccine and broadbased antiviral therapeutic development has demonstrated the strong potential of the FIV model for investigation of novel vaccine approaches and antiviral drugs. Lastly, similarities observed for host cell targets for virus infection and immunodeficiencies associated with FIV and HIV infection in vivo offer strong support for use of this animal model for crucial studies focused on mechanisms of immunopathogenesis of lentivirus-induced acquired immunodeficiency.

ACKNOWLEDGMENTs. We are grateful for our FIV research colleagues and collaborators who have partsicipated in the development of the FIV animal model and who have provided helpful input or references.

\section{REFERENCES}

1. N. C. Pedersen, E. W. Ho, M. L. Brown, and J. K. Yamamoto, Isolation of a Tlymphotropic virus from domestic cats with an immunodeficiency-like syndrome, Science 235, 790-793 (1987).

2. J. K. Yamamoto, E. Sparger, E. W. Ho, P. R. Andersen, T. P. O'Connor, C. P. Mandell, L. Lowenstine, R. Munn, and N. C. Pedersen, Pathogenesis of experimentally induced feline immunodeficiency virus infection in cats, Am. J. Vet. Res. 49, 1246-1258 (1988).

3. P. J. Brown, C. D. Hopper, and D. A. Harbour, Pathological features of lymphoid tissues in cats with natural feline immunodeficiency virus infection, J. Comp. Path. 104, 345-355 (1991).

4. A. M. Beebe, N. Dua, T. G. Gluckstern, P. Moore, N. C. Pedersen, and S. Dandekar, The primary stage of feline immunodeficiency virus infection: viral dissemination and cellular targets, J. Virol. 68, 3080-3091 (1994).

5. C. D. Ackley, J. K. Yamamoto, N. Levy, N. C. Pedersen, and M. D. Cooper, Immunologic abnormalities in pathogen-free cats experimentally infected with feline immunodeficiency virus, J. Virol. 64, 5652-5655 (1990).

6. J. E. Barlough, C. D. Ackley, J. W. George, N. Levy, R. Acevedo, P. F. Moore, B. A. Rideout, M. D. Cooper, and N. C. Pedersen, Acquired immune dysfunction in cats with experimentally induced feline immunodeficiency virus infection: comparison of short-term and long-term infections, J.A.I. D. S. 4, 219-227 (1991).

7. L. J. Diehl, C. K. Mathiason-Dubard, L. L. O’Neil, L. A. Obert, and E. A. Hoover, Induction of accelerated feline immunodeficiency virus disease by acute-phase virus passage, J. Virol. 69, 6149-6157 (1995).

8. R. V. English, P. Nelson, C. M. Johnson, M. Nasisse, W. A. Tompkins, and M. B. Tompkins, Development of clinical disease in cats experimentally infected with feline immunodeficiency virus, J. Infect. Dis. 170, 543-552 (1994).

9. T. Ishida and I. Tomoda, Clinical staging of feline immunodeficiency virus infection, Nippon Juigaku Zasshi. 52, 645-648 (1990). 
10. C. Novotney, R. V. English, J. Housman, M. G. Davidson, M. P. Nasisse, C. Jeng, W. C. Davis, and M. B. Tompkins, Lymphocyte population changes in cats naturally infected with feline immunodeficiency virus, AIDS 4, 1213-1218 (1990).

11. N. C. Pedersen, C. M. Leutenegger, J. Woo, and J. Higgins, Virulence differences between two field isolates of feline immunodeficiency virus (FIV-A Petaluma and FIV-CPGammar) in young adult specific pathogen free cats, Vet. Immunol. Immunopathol. 79, 53-67 (2001).

12. B. A. Rideout, L. J. Lowestine, C. A. Hutson, P. F. Moore, and N. C. Pedersen, Characterization of morphological changes and lymphocytes subset distribution in lymph nodes from cats with naturally acquired feline immunodeficiency virus infection, Vet. Immunol. Immunopathol. 29, 391-399 (1992).

13. J. K. Yamamoto, H. Hansen, E. W. Ho, T. Y. Morishita, T. Okuda, T. R. Sawa, R. M. Nakamura, and N. C. Pedersen, Epidemiologic and clinical aspects of feline immunodeficiency virus infection in cats from the continental United States and Canada and possible mode of transmission, J. Am. Vet. Med. Assoc. 194, 213-220 (1989).

14. M. Bendinelli, M. Pistello, S. Lombari, A. Poli, C. Garzelli, D. Matteucci, L. CeccheriniNelli, G. Malvaldi, and F. Tozzini, Feline immunodeficiency virus: an interesting model for AIDS studies and an important cat pathogen, Clin. Micorbiol. Rev. 8, 87-112 (1995).

15. T. Ishida, T. Washizu, K. Toriyabe, S. Motoyoshi, I. Tomoda, and N. Pedersen, Feline immunodeficiency virus infection in cats of Japan, J. Am. Vet. Med. Assoc. 194, 221-225 (1989).

16. M. J. Burkhard, L. A. Obert, L. L. O'Neil, L. J. Diehl, and E. A. Hoover, Mucosal transmission of cell-associated and cell-free feline immunodeficiency virus, AIDS Res. Hum. Retroviruses. 13, 347-355 (1997).

17. M. J. Burkhard and G. A. Dean, Transmission and immunopathogenesis of FIV in cats as a model for HIV, Curr. HIV Res. 1, 15-29 (2003).

18. S. A. Bishop, C. R. Stokes, T. J. Gruffydd-Jones, C. V. Whiting, and D. A. Harbour, Vaginal and rectal infection of cats with feline immunodeficiency virus, Vet. Microbiol. 51, 217-227 (1996).

19. L. L. O'Neil, M. J. Burkhard, L. J. Diehl, and E. A. Hoover, Vertical transmission of feline immunodeficiency virus, AIDS Res. Hum. Retroviruses 11, 171-182 (1995).

20. L. L. O'Neil, M. J. Burkhard, and E. A. Hoover, Frequent perinatal transmission of feline immunodeficiency virus by chronically infected cats, J. Virol. 70, 2894-2901 (1996).

21. L. A. Obert and E. A. Hoover, Feline immunodeficiency virus clade C mucosal transmission and disease courses, AIDS Res. Hum. Retroviruses 16, 677-688 (2000).

22. L. A. Obert and E. A. Hoover, Relationship of lymphoid lesions to disease course in mucosal feline immunodeficiency virus type C infection, Vet. Pathol. 37, 386-401 (2000).

23. L. A. Obert and E. A. Hoover, Early pathogenesis of transmucosal feline immunodeficiency virus infection, J. Virol. 76, 6311-6322 (2002).

24. O. J. Cohen and A. S. Fauci, in Fields Virology, edited by D. M. Knipe and P. M. Howley (Lippincott Williams \& Wilkins, Philadelphia, PA, 2001), 2043-2094.

25. T. S. Tochikura, K. A. Hayes, C. M. Cheney, T. A. Tanabe, J. L. Rojko, L. E. Mathes, and R. G. Olsen, In vitro replication and cytopathogenicity of the feline immunodeficiency virus for feline T4 thymic lymphoma 3201 cells, Virology 179, 492-497 (1990).

26. J. Goff, in Fields Virology, edited by D. M. Knipe and P. M. Howley (Lippincott Williams \& Wilkins, Philadelphia, 2001), 1871-1939.

27. R. Steinman, J. Dombrowski, T. O’Connor, R. C. Montelaro, Q. Tonelli, K. Lawrence, C. Seymour, J. Goodness, N. C. Pedersen, and P. R. Andersen, Biochemical and immunological characterization of the major structural proteins of feline immunodeficiency virus, J. Gen. Virol. 71, 701-706 (1990).

28. H. F. Egberink, J. Ederveen, R. C. Montelaro, N. C. Pedersen, M. C. Horzinek, and M. J. Koolen, Intracellular proteins of feline immunodeficiency virus and their antigenic relationship with equine infectious anaemia virus proteins, J. Gen. Virol. 71, 739-743 (1990). 
29. P. C. Wagaman, C. S. Hasselkus-Light, M. Henson, D. L. Lerner, T. R. Phillips, and J. H. Elder, Molecular cloning and characterization of deoxyuridine triphosphatase from feline immunodeficiency virus (FIV), Virology 196, 451-457 (1993).

30. J. H. Elder, D. L. Lerner, C. S. Hasselkus-Light, D. J. Fontenot, E. Hunter, P. A. Luciw, R. C. Montelaro, and T. R. Phillips, Distinct subsets of retroviruses encode dUTPase, J. Virol. 66, 1791-1794 (1992).

31. J. Coffin, in Fundamental Virology, edited by B. N. Fields and K. M. Knipe (Raven Press, New York, 1991), 645-708.

32. M. Shimojima, T. Miyazawa, Y. Ikeda, E. L. McMonagle, H. Haining, H. Akashi, Y. Takeuchi, M. J. Hosie, and B. J. Willett, Use of CD134 as a primary receptor by the feline immunodeficiency virus, Science 303, 1192-1195 (2004).

33. B. J. Willett, L. Picard, M. J. Hosie, J. D. Turner, K. Adema, and P. R. Clapham, Shared usage of the chemokine receptor CXCR4 by the feline and human immunodeficiency viruses, J. Virol. 71, 6407-6415 (1997).

34. S. Giannecchini, F. Bonci, M. Pistello, D. Matteucci, O. Sichi, P. Rovero, and M. Bendinelli, The membrane-proximal tryptophan-rich region in the transmembrane glycoprotein ectodomain of feline immunodeficiency virus is important for cell entry, Virology 320, 156-166 (2004).

35. A. P. De Parseval, S. V. Su, J. H. Elder, and B. Lee, Specific interaction of feline immunodeficiency virus surface glycoprotein with human DC-SIGN, J. Virol. 78, 2597-2600 (2004).

36. A. P. De Parseval and J. H. Elder, Binding of recombinant feline immunodeficiency virus surface glycoprotein to feline cells: role of CXCR4, cell-surface heparans, and an unidentified non-CXCR4 receptor, J. Virol. 75, 4528-4539 (2001).

37. R. A. Olmsted, V. M. Hirsch, R. H. Purcell, and P. R. Johnson, Nucleotide sequence analysis of feline immunodeficiency virus: genome organization and relationship to other lentiviruses, Proc. Natl. Acad. Sci. USA 86, 8088-8092 (1989).

38. R. L. Talbott, E. E. Sparger, K. M. Lovelace, W. M. Fitch, N. C. Pedersen, P. A. Luciw, and J. H. Elder, Nucleotide sequence and genomic organization of feline immunodeficiency virus, Proc. Natl. Acad. Sci. USA 86, 5743-5747 (1989).

39. F. J. Thompson, J. Elder, and J. C. Neil, Cis- and trans-regulation of feline immunodeficiency virus: identification of functional binding sites in the long terminal repeat, J. Gen. Virol. 75, 545-554 (1994).

40. E. E. Sparger, B. L. Shacklett, L. Renshaw-Gegg, P. A. Barry, N. C. Pedersen, J. H. Elder, and P. A. Luciw, Regulation of gene expression directed by the long terminal repeat of the feline immunodeficiency virus, Virology 187, 165-177 (1992).

41. T. R. Phillips, C. Lamont, D. A. M. Konings, B. L. Schacklett, C. A. Hamson, P. A. Luciw, and $\mathrm{J}$. H. Elder, Identification of the rev transactivation and rev-responsive elements of feline immunodeficiency virus, J. Virol. 66, 5464-5471 (1992).

42. T. R. Phillips, R. L. Talbott, C. Lamont, S. Muir, K. Lovelace, and J. H. Elder, Comparison of two host cell range variants of feline immunodeficiency virus, J. Virol. 64, 4605-4613 (1990).

43. T. Miyazawa, M. Kohmoto, Y. Kawaguchi, K. Tomonaga, T. Toysaki, K. Kiuta, A. Adachi, and T. Mikami, The AP-1 binding site in the feline immunodeficiency virus long terminal repeat is not required for virus replication in feline T lymphocytes, J. Gen. Virol. 74, 1573-1580 (1993).

44. M. T. Browning, F. Mustafa, R. D. Schmidt, K. A. Lew, and T. A. Rizvi, Delineation of sequences important for efficient packaging of feline immunodeficiency virus RNA, J. Gen. Virol. 84, 621-627 (2003).

45. I. Kemler, R. Barraza, and E. M. Poeschla, Mapping the encapsidation determinants of feline immunodeficiency virus, J. Virol. 76, 11889-11903 (2002).

46. T. Whitwam, M. Peretz, and E. Poeschla, Identification of a central DNA flap in feline immunodeficiency virus, J. Virol. 75, 9407-9414 (2001). 
47. L. Bigornia, K. M. Lockridge, and E. E. Sparger, Construction and in vitro characterization of attenuated FIV LTR mutant viruses, J. Virol. 75, 1054-1060 (2001).

48. Y. Inoshima, Y. Miyazawa, and T. Mikami, In vivo functions of the auxiliary genes and regulatory elements of feline immunodeficiency virus, Vet. Microbiol. 60, 141-153 (1998).

49. Y. Kawaguchi, K. Tomonaga, K. Maeda, M. Ono, T. Miyazawa, M. Kohmoto, T. Y., and T. Mikami, The C/EBP site in the feline immunodeficiency virus (FIV) long terminal repeart (LTR) is necessary for its efficient replication and is also involved in the inhibition of FIV LTR-directed gene expression by pseudorabies virus ICP4, Virology 208, 492-499 (1995).

50. Y. Ikeda, Y. Inoshima, Y. Kawaguchi, K. Maeda, M. Kohmoto, C. Kai, T. Miyazawa, and T. Mikami, Protein-binding properties of the putative AP-1 and ATF sequence in the feline immunodeficiency virus long terminal repeat, J. Gen. Virol. 79, 95-99 (1998).

51. B. R. Cullen and W. C. Greene, Regulatory pathways governing HIV-1 replication, Cell 58, 423-426 (1989).

52. B. R. Cullen, Human immunodeficiency virus as a protoypic complex retrovirus, J. Virol. 65, 1053-1056 (1991).

53. D. Derse, Bovine leukemia virus transcription is controlled by a virus-encoded transactiving factor and by cis-acting response elements, J. Virol. 61, 2462-2471 (1987).

54. R. P. S. Kwok, M. E. Laurance, J. R. Lundblad, P. S. Goldman, H. M. Shih, L. M. Connor, S. J. Marriott, and R. H. Goodman, Control of cAMP-regulated enhancers by the viral transctivator Tax through CREB and the co-activator CBP, Nature 380, 642-646 (1996).

55. L. Willems, R. Kettmann, G. Chen, D. Portetelle, A. Burny, and D. Derse, A cyclic AMPresponsive DNA-binding protein (CREB2) is a cellular transactivator of the bovine leukemia virus long terminal repeat, J. Virol. 66, 766-772 (1992).

56. L. J. Zhao and C. Z. Giam, Interaction of the human T-cell lymphotropic virus type 1 (HTLV-1) transcriptional activator Tax with cellular factors that bind specifically to the 21-base-pair repeats in the HTLV-1 enhancer, Proc. Natl. Acad. Sci. USA 88, 11445-11449 (1991).

57. B. A. Morse, L. M. Carruth, and J. E. Clements, Targeting of the visna virus tat protein to AP-1 sites: interactions with the bZIP domains of fos and jun in vitro and in vivo, J. Virol. 73, 37-45 (1999).

58. J. L. Hess, J. A. Small, and J. E. Clements, Sequences in the visna virus long terminal repeat that control transciptional activity and respond to viral trans-activation: involvement of AP-1 sites in basal activity and trans-activation, J. Virol. 63, 3001-3015 (1989).

59. L. M. Carruth, B. A. Morse, and J. E. Clements, The leucine domain of the visna virus tat protein mediates targeting to an AP-1 site in the viral long terminal repeat, J. Virol. 70, 4338-4344 (1996).

60. K. Tomonaga and T. Mikami, Molecular biology of the feline immunodeficiency virus auxiliary genes, J. Gen. Virol. 77, 1611-1621 (1996).

61. A. K. Waters, A. P. De Parseval, D. L. Lerner, J. C. Neil, F. J. Thompson, and J. H. Elder, Influence of ORF2 on host cell tropism of feline immunodeficiency virus, Virology 215, 10-16 (1996).

62. C. Nueveut, R. Vigne, J. E. Clements, and J. Sire, The visna transcriptional activator TAT: effects on the viral LTR and on cellular genes, Virology 197, 236-244 (1993).

63. S. Villet-Hoc, C. Faure, B. Bouzar, G. Verdier, Y. Chelbloune, and C. Legras, Tat proteins of caprine arthritis encephalitis (CAEV) and maedi visna (MV) viruses are not transactivator proteins of MVV and CAEV LTRs, American Society of Virology Conference (University of Kentucky). 21st annual meeting, 166 (2002).

64. A. Gatignol, Identification of cellular proteins that bind to the human immunodeficiency virus type 1 trans-activation-responsive TAR element, Proc. Natl. Acad. Sci. USA 86, 7828-7832 (1989). 
65. R. Marciniak, M. Garcia-Blanco, and P. Sharp, Identification and characterization of a HeLa nuclear protein that specifically binds to the trans-activation-response (TAR) element of human immunodeficiency virus, Proc. Natl. Acad. Sci. USA 87, 3624-3628 (1990).

66. A. P. De Parseval and J. H. Elder, Demonstration that orf2 encodes the feline immunodefiency virus tranactivating (Tat) protein and characterization of a unique gene product with partial rev activity, J. Virol. 73, 608-617 (1999).

67. U. Chatterji, A. P. De Parseval, and J. H. Elder, Feline immunodeficiency virus Orf-A is distinct from other lentivirus transactivators, J. Virol. 76, 9624-9634 (2002).

68. K. Tomonaga, Y. Shin, M. Fukasawa, T. Miyazawa, A. Adachi, and T. Mikami, Feline immunodeficiency virus gene expression: analysis of the RNA splicing pattern and the monocistronic rev mRNA, J. Gen. Virol. 74, 2409-2417 (1993).

69. T. Kiyomasu, T. Miyazawa, T. Furuya, R. Shibata, H. Sakai, J. Sakuragi, M. Fukasawa, N. Maki, A. Hasegawa, T. Mikami, and A. Adachi, Identification of feline immunodeficiency virus rev gene activity, J. Virol. 65, 4539-4542 (1991).

70. M. L. Manrique, C. C. P. Celma, S. A. Gonzalez, and J. L. Affranchino, Mutational analysis of the feline immunodeficiency virus matrix protein, Virus Res. 76, 103-113 (2001).

71. J. H. Elder, M. Schnolzer, C. S. Hasselkus-Light, M. Henson, D. A. Lerner, T. R. Phillips, P. C. Wagaman, and S. B. H. Kent, Identification of proteolytic processing sites within the Gag and Pol polyproteins of feline immunodeficiency virus, J. Virol. 67, 1869-1876 (1993).

72. R. Swanstrom and J. W. Wills, in Retroviruses, edited by J. Coffin, S. H. Hughes and H. E. Varmus (Cold Spring Harbor Laboratory Press, Cold Spring Harbor, NY, 1997), 263-334.

73. E. O. Freed and M. A. Martin, in Fields Virology, edited by D. M. Knipe and P. M. Howley (Lippincott Williams \& Wilkins, Philadephia, PA, 2001), 1971-2041.

74. T. L. South, P. R. Blake, R. C. Sowder, L. O. Arthur, L. E. Henderson, and M. F. Summers, The nucleocapsid protein isolated from HIV-1 particles binds zinc and forms retroviraltype zinc fingers, Biochemistry 29, 7786-7789 (1990).

75. M. Moscardini, M. Pistello, M. Bendinelli, D. Ficheux, J. T. Miller, C. Gabus, S. F. Le Grice, W. K. Surewicz, and J. L. Darlix, Functional interactions of nucleocapsid protein of feline immunodeficiency virus and cellular prion protein with the viral RNA, J. Mol. Biol. 318, 149-159 (2002).

76. E. O. Freed, Viral late domains, J. Virol. 76, 4679-4687 (2002).

77. H. G. Gottlinger, T. Dorfman, J. G. Sodroski, and W. A. Haseltine, Effect of mutations affecting the p6 gag protein on human immunodeficiency virus particle release, Proc. Natl. Acad. Sci. USA 88, 3195-3199 (1991).

78. M. Huang, J. Orenstein, M. Martin, and E. Freed, p6Gag is required for particle production from full-length human immunodeficiency virus type 1 molecular clones expressing protease, J. Virol. 69, 6810-6818 (1995).

79. M. Sudol, Structure and function of the WW domain, Prog. Biophys. Molec. Biol. 65, 113-132 (1996).

80. D. G. Demirov, A. Ono, J. M. Orenstein, and E. O. Freed, Overexpression of the Nterminal domain of TSF101 inhibits HIV-1 budding by blocking late domain function, Proc. Natl. Acad. Sci. USA 99, 955-960 (2002).

81. J. E. Garrus, U. K. von Schwedler, O. W. Pornillos, S. G. Morham, K. H. Zavitz, H. E. Wang, D. A. Wettstein, K. M. Stray, M. Cote, R. L. Rich, and W. I. Sundquist, Tsg101 and the vacuolar protein sorting pathway are essential for HIV-1 budding, Cell 107, 55-65 (2001).

82. J. Martin-Serrano, T. Zang, and P. D. Bieniasz, HIV-1 and Ebola virus encode small peptide motifs that recruit Tsg101 to sites of particle assembly to facilitate egress, Nat. Med. 7, 1313-1319 (2001). 
83. X. Guo, J. Hu, J. B. Whitney, R. S. Russell, and C. Liang, Important role for the CA-NC spacer region in the assembly of bovine immunodeficiency virus Gag protein, J. Virol. $78,551-560$ (2004).

84. H. G. Krausslich, M. Facke, A. M. Heuser, J. Konvalinka, and H. Zentgraf, The spacer peptide between human immunodeficiency virus capsid and nucleoscapsid proteins is essential for ordered assembly and viral infectivity, J. Virol. 69, 3407-3419 (1995).

85. M. A. Accola, S. Hoglund, and H. G. Gottlinger, A putative a-helical structure which overlaps the capsid-p2 boundary in the human immunodeficiency virus type 1 Gag precursor is crucial for viral particle assembly, J. Virol. 72, 2071-2078 (1998).

86. S. Morikawa and D. H. L. Bishop, Identification and analysis of the gag-pol ribosomal frameshift site of feline immunodeficiency virus, Virology 186, 389-397 (1992).

87. S. Morikawa, T. F. Booth, and D. H. L. Bishop, Analyses of the requirements for the synthesis of virus-like particles by feline immunodeficiency virus Gag using baculovirus vectors, Virology 183, 288-297 (1991).

88. K. von der Helm, Retroviral proteases: structure, function and inhibition from a nonanticipated viral enzyme to the target of a most promising HIV therapy, Biol. Chem. 377, 765-774 (1996).

89. G. S. Laco, C. Schalk-Hihi, J. Lubkowski, G. M. Morris, A. Zdanov, A. Olson, J. H. Elder, A. Wlodawer, and A. Gustchina, Crystal structures of the inactive D30N mutant of feline immunodeficiency virus protease complexed with a substrate and an inhibitor, Biochemistry 36, 10696-10708 (1997).

90. A. Wlodawer, A. Gustchina, L. Reshetnikova, J. Lubkowski, A. Zdanov, K. Y. Hui, E. L. Angleton, W. G. Farmerie, M. M. Goodenow, and D. Bhatt, Structure of an inhibitor complex of the proteinase from feline immunodeficiency virus, Nat. Struct. Biol. 2, 480-488 (1995).

91. M. Schnolzer, H. R. Rackwitz, A. Gustchina, G. S. Laco, A. Wlodawer, J. H. Elder, and S. B. Kent, Comparative properties of feline immunodeficiency virus (FIV) and human immunodeficiency virus type 1 (HIV-1) proteinases prepared by total chemical synthesis., Virology 224, 268-275 (1996).

92. Y. Lin, Z. Q. Beck, T. Lee, V. Le, G. M. Morris, A. J. Olson, C. Wong, and J. H. Elder, Alteration of substrate and inhibitor specificity of feline immunodeficiency virus protease, J. Virol. 74, 4710-4720 (2000).

93. T. Lee, G. S. Laco, B. E. Torbett, H. S. Fox, D. L. Lerner, J. H. Elder, and C. H. Wong, Analysis of the S3 and S3' subsite specificities of feline immunodeficiency virus (FIV) protease: development of a broad-based protease inhibitor efficacious against FIV, SIV, and HIV in vitro and ex vivo, Proc. Natl. Acad. Sci. USA 95, 939-944 (1998).

94. Z. Q. Beck, Y. Lin, and J. H. Elder, Molecular basis for the relative substrate specificity of human immunodeficiency virus type 1 and feline immunodeficiency virus proteases, J. Virol. 75, 9458-9469 (2001).

95. Y. C. Lin, Z. Q. Beck, G. M. Morris, A. J. Olson, and J. H. Elder, Structural basis for distinctions between substrate and inhibitor specificities for feline immunodeficiency virus and human immunodeficiency virus proteases, J. Virol. 77, 6589-6600 (2003).

96. J. Kervinen, J. Lubkowski, A. Zdanov, D. Bhatt, B. M. Dunn, K. Y. Hui, D. J. Powell, J. Kay, A. Wlodawer, and A. Gustchina, Toward a universal inhibitor of retroviral proteases: comparative analysis of the interactions of LP-130 complexed with proteases from HIV1, FIV, and EIAV, Protein Sci. 7, 2314-2323 (1998).

97. B. Buhler, Y. Lin, G. M. Morris, O. A. J., C. Wong, D. D. RIchman, J. H. Elder, and B. E. Torbett, Viral evolution in response to the broad-based retroviral protease inhibitor TL-3, J. Virol. 75, 9502-9508 (2001).

98. M. Hottiger and U. Hubscher, Human immunodeficiency virus type 1 reverse transcriptase, Biol. Chem. Hopppe Seyler 377, 97-120 (1996). 
99. T. W. North, G. L. North, and N. C. Pedersen, Feline immunodeficiency virus, a model for reverse transcriptase-targeted chemotherapy for acquired immune deficiency syndrome, Antimicrob. Agents Chemother. 33, 915-919 (1989).

100. T. W. North, R. C. Cronn, K. M. Remington, R. T. Tandberg, and R. C. Judd, Characterization of reverse transcriptase from feline immunodeficiency virus, J. Biol. Chem. 265, 5121-5128 (1990).

101. M. Amacker, M. Hottiger, and U. Hubscher, Feline immunodeficiency virus reverse transcritpase: expression, functional characterization, and reconstitution of the 66- and 51kilodalton subunits, J. Virol. 69, 6273-6279 (1995).

102. R. C. Cronn, J. D. Whitmer, and T. W. North, RNase H activity associated with reverse transcriptase from feline immunodeficiency virus, J. Virol. 66, 1215-1218 (1992).

103. G. J. Klarmann, R. A. Smith, R. F. Schinazi, T. W. North, and B. D. Preston, Site-specific incorporation of nucleoside analogs by HIV-1 reverse transcriptase and the template grip mutant P157S, J. Biol. Chem. 275, 359-366 (2000).

104. K. M. Remington, B. Chesebro, K. Wehrly, N. C. Pedersen, and T. W. North, Mutants of feline immunodeficiency virus resistant to 3 '-azido- $3^{\prime}$-deoxythymidine, J. Virol. 65 , 308-312 (1991).

105. K. M. Remington, Y.-Q. Zhu, T. R. Phillips, and T. W. North, Rapid phenotypic reversion of zidovudine-resistant feline immunodeficiency virus without loss of drug-resistant feline immunodeficiency virus without loss of drug-resistant reverse transcriptase, J. Virol. 68, 632-637 (1994).

106. R. A. Smith, K. M. Remington, R. M. Lloyd, R. F. Schinazi, and T. W. North, A novel Met-to-Thr mutation in the YMDD motif of reverse transcriptase from feline immunodeficiency virus confers resistance to oxathiolane nucleosides, J. Virol. 71, 2357-2362 (1997).

107. S. M. A. McCrackin and D. G. McBroom, In vitro characterization of FIV-pPPR, a pathogenic molecular clone of feline immunodeficiency virus, and two drug-resistant pol gene mutants, Am J. Vet. Res. 62, 588-594 (2001).

108. R. A. Smith, K. M. Remington, B. D. Preston, R. F. Schinazi, and T. W. North, A novel point mutation at position 156 of reverse transcriptase from feline immunodeficiency virus confers resistance to the combination of (-)-beta- $2^{\prime}, 3^{\prime}$-dideoxy- $3^{\prime}$-thiacytidine and 3'-azido-3'-deoxythymidine, J. Virol. 72, 2335-2340 (1998).

109. J. Auwerx, T. W. North, B. D. Preston, G. J. Klarmann, E. de Clercq, and J. Balzarini, Chimeric human immunodeficiency virus type 1 and feline immunodeficiency virus reverse transcriptases: role of the subunits in resistance/sensitivity to non-nucleoside reverse transcriptase inhibitors, Mol. Pharmacol. 61, 400-406 (2002).

110. M. Amacker and U. Hubscher, Chimeric HIV-1 and feline immunodeficiency virus reverse transcriptases: critical role of the p51 subunit in the structural integrity of heterodimeric lentiviral DNA polymerases, J. Mol. Biol. 278, 757-765 (1998).

111. J. E. Barlough, T. W. North, C. L. Oxford, K. M. Remington, S. Dandekar, M. N. Ellis, and N. C. Pedersen, Feline immunodeficiency virus infection of cats as a model to test the effect of certain in vitro selection pressures on the infectivity and virulence of resultant lentivirus variants, Antiviral Res. 22, 259-272 (1993).

112. M. H. Gadsen, E. M. McIntosh, J. C. Game, P. J. Wilson, and R. H. Haynes, dUTP pyrophosphatase is an essential enzyme in Saccharomyces cerevisiae, EMBO J. 12, 4425-4431 (1993).

113. S. L. Payne and J. H. Elder, The role of retroviral dUTPases in replication and virulence, Curr. Protein Pept. Sci. 2, 381-388 (2001).

114. D. L. Lerner, P. C. Wagaman, T. Phillips, O. Prospero-Garcia, S. J. Henriksen, H. S. Fox, F. E. Bloom, and J. H. Elder, Increased mutation frequency of feline immunodeficiency virus lacking functional deoxyuridine-triphosphatase, Proc. Natl. Acad. Sci, USA 92, 7480-7484 (1995). 
115. W. K. Steagall, M. D. Robek, S. T. Perry, F. J. Fuller, and S. L. Payne, Incorporation of uracil into viral DNA correlates with reduced replication of EIAV in macrophages, Virology 210, 302-313 (1995).

116. L. Selig, S. Benichou, M. E. Rogel, L. I. Wu, M. A. Vodicka, J. Sire, R. Benarous, and M. Emerman, Uracil DNA glycosylase specifically interacts with Vpr of both human immunodeficiency virus type 1 and simian immunodeficiency virus of sooty mangabeys, but binding does not correlate with cell cycle arrest, J. Virol. 71, 4842-4846 (1997).

117. L. M. Mansky, S. Preveral, L. Selig, R. Benarous, and S. Benichou, The interaction of Vpr with uracil DNA glycosylase modulates the human immunodeficiency virus type 1 in vivo mutation rate, J. Virol. 74, 7039-7047 (2000).

118. L. M. Mansky, E. Le Rouzic, S. Benichou, and L. C. Gajary, Influence of reverse transcriptase variants, drugs, and $\mathrm{Vpr}$ on human immunodeficiency virus type 1 mutant frequencies, J. Virol. 77, 2071-2080 (2003).

119. M. Bouhamdan, S. Benichou, F. Rey, J. Navarro, I. Agostini, B. Spire, J. Camonis, G. Slupphaug, R. Vigne, R. Benarous, and J. Sire, Human immunodeficiency virus type 1 Vpr protein binds to the uracil DNA glycosylase DNA repair enzyme, J. Virol. 70, 697-704 (1996).

120. P. O. Brown, in Retroviruses, edited by J. M. Coffin, S. H. Hughes, and H. E. Varmus (Cold Spring Harbor Laboratory Press, Cold Spring Harbor, NY, 1997), 161-204.

121. C. Vink, K. H. Van Der Linden, and R. H. A. Plasterk, Activities of the feline immunodeficiency virus integrase protein produced in Escherichia coli, J. Virol. 68, 1468-1474 (1994).

122. E. Khan, J. P. Mack, R. A. Katz, J. Kulkosky, and A. M. Skalka, Retroviral integrase domains: DNA binding and the recognition of LTR sequences, Nucleic Acids Res. 19, 851-860 (1991).

123. Y. Shibagaki and S. A. Chow, Central core domain of retroviral integrase is responsible for target site selection, J. Biol. Chem. 272, 8361-8369 (1997).

124. Y. Shibagaki, M. L. Holmes, R. S. Appa, and S. A. Chow, Characterization of feline immunodeficiency virus integrase and analysis of functional domains, Virology 230, 1-10 (1997).

125. D. T. Saenz, N. Loewen, M. Peretz, T. Whitwam, R. Barraza, K. G. Howell, J. M. Holmes, M. Good, and E. M. Poeschla, Unintegrated lentivirus DNA persistence and accessibility to expression in nondividing cells: analysis with class I integrase mutants, J. Virol. 78, 2906-2920 (2004).

126. R. A. Fouchier and M. H. Malim, Nuclear import of human immunodeficiency virus type-1 preintegration complexes, Adv. Virus Res. 52, 275-299 (1999).

127. B. Bouyac, M., J. D. Dvorin, R. A. Fouchier, Y. Jenkins, B. E. Meyer, L. I. Wu, M. Emerman, and M. H. Malim, HIV-1 infection requires a functional integrase NLS, Cell 7, 1025-1035 (2001).

128. C. L. Woodward, Y. Wang, W. J. Dixon, H. Htun, and S. A. Chow, Subcellular localization of feline immunodeficiency virus integrase and mapping of its karyophiclic determinant, J. Virol. 77, 4516-4527 (2003).

129. E. B. Stephens, E. Monck, K. Reppas, and E. J. Butfiloski, Processing of the glycoprotein of feline immunodeficiency virus: effect of inhibitors of glycosylation, J. Virol. 65, 1114-1123 (1991).

130. E. J. Verschoor, E. G. J. Hulskotte, J. Ederveen, M. J. M. Koolen, M. C. Horzinek, and P. J. M. Rottier, Post-translational processing of the feline immunodeficiency virus envelope precursor protein, Virology 193, 433-438 (1993).

131. M. L. Poss, S. W. Dow, and E. A. Hoover, Cell-specific envelope glycosylation distinguishes FIV glycoproteins produced in cytopathically and noncytopathically infected cells, Virology 188, 25-32 (1992). 
132. T. W. Vahlenkamp, A. De Ronde, P. J. M. Rottier, M. C. Horzinek, H. F. Egberink, and E. J. Verschoor, The feline immunodeficiency virus envelope protein precursor: functional analysis of a leader deletion mutant, Vet. Microbiol. 69, 115-116 (1999).

133. G. Pancino, L. Camoin, and P. Sonigo, Structural analysis of the principal immunodominant domain of the feline immunodeficieny virus transmembrane glycoprotein, J. Virol. 69, 2110-2118 (1995).

134. G. Pancino, I. Fossati, C. Chapey, S. Castelot, B. Hurtrel, A. Maraillon, D. Katzmann, and P. Sonigo, Structure and variations of feline immunodeficiency virus envelope glycoproteins, Virology 192, 659-662 (1993).

135. P. F. Serres, Molecular mimicry betwen the trimeric ectodomain of the transmembrane protein of immunosuppresive lentiviruses (HIV-SIV-FIV) and interleukin 2, C. R. Acad. Sci. Ser III. 323, 1019-1029 (2000).

136. G. Pancino and P. Sonigo, Retention of viral infectivity after extensive mutation of the highly conserved immunodominant domain of the feline immunodeficiency virus envelope, J. Virol. 71, 4339-4346 (1997).

137. H. Garg, F. J. Fuller, and W. A. F. Tompkins, Mechanism of feline immunodeficiency virus envelope glycoprotein-mediated fusion, Virology 321, 274-286 (2004).

138. S. Giannecchini, A. Di Fenza, A. M. D'Ursi, D. Matteucci, P. Rovero, and M. Bendinelli, Antiviral activity and conformational features of an octapeptide derived from the membrane-proximal ectodomain of the feline immunodeficiency virus transmembrane glycoprotein, J. Virol. 77, 3724-3733 (2003).

139. K. Salzwedel, J. T. West, and E. Hunter, A conserved tryptophan-rich motif in the membrane-proximal region of the human immunodeficiency virus type 1 gp41 ectodomain is important for Env-mediated fusion and virus infectivity, J. Virol. 73, 2469-2480 (1999).

140. I. Munoz-Barroso, K. Salzwedel, E. Hunter, and R. Blumenthal, Role of the membraneproximal domain in the initial stages of human immunodeficiency virus-1 envelope glycoprotein-mediated membrane fusion, J. Virol. 73, 6089-6092 (1999).

141. M. A. Rigby, E. C. Holmes, M. Pistello, A. Mackay, A. J. L. Brown, and J. C. Neil, Evolution of structural proteins of feline immunodeficiency virus: molecular epidemiology and evidence of selection for change, J. Gen. Virol. 74, 425-436 (1993).

142. D. L. Sodora, E. G. Shpaer, B. E. Kitchell, S. W. Dow, E. A. Hoover, and J. I. Mullins, Identification of three feline immunodeficiency virus env gene subtypes and comparison of evolutionary patterns between FIV and HIV-1, J. Virol. 68, 2230-2238 (1994).

143. W. K. Greene, J. Meers, G. del Fierro, P. R. Carnegie, and W. F. Robinson, Extensive sequence variation of feline immunodeficiency virus env gene in isolates from naturally infected cats, Arch. Virol. 133, 51-62 (1993).

144. M. G. Pistello, E. Cammarota, E. Nicoletti, D. Matteucci, M. Curcio, D. Del Mauro, and M. Bendinelli, Analysis of the genetic diversity and phylogenetic relationship of Italian isolates of feline immunodeficiency virus indicates a high prevalence and heterogeneity of subtype B, J. Gen. Virol. 78, 2247-2257 (1997).

145. M. R. Pecoraro, K. Tomonaga, T. Miyazawa, Y. Kawaguchi, S. Sugita, Y. Tohya, C. Kai, M. E. Etcheverrigaray, and T. Mikami, Genetic diversity of Argentine isolates of feline immunodeficiency virus, J. Gen. Virol. 77, 2031-2035 (1996).

146. A. Steinrigl and D. Klein, Phylogenetic analysis of feline immunodeficiency virus in Central Europe: a prerequisite for vaccination and molecular diagnostics, J. Gen. Virol. 84, 1301-1307 (2003).

147. S. Kakinuma, K. Motokawa, T. Hohdatsu, J. K. Yamamoto, H. Koyama, and H. Hashimoto, Nucleotide sequence of feline immunodeficiency virus: classification of Japanese isolates into two subtypes which are distinct from non-Japanese subtypes, J. Virol. 69, 3639-3646 (1995). 
148. G. Inada, T. Miyazawa, Y. Inoshima, M. Kohmoto, Y. Ikeda, C. H. Liu, J. A. Lin, T. F. Kuo, and T. Mikami, Phylogenetic analysis of feline immunodeficiency virus isolated from cats in Taiwan, Arch. Virol. 142, 1456-1459 (1997).

149. M. A. Carpenter, E. W. Brown, D. W. MacDonald, and S. J. O’Brien, Phylogeographic patterns of feline immunodeficiency virus genetic diversity in the domestic cat, Virology 251, 234-243 (1998).

150. M. H. Bachmann, C. K. Mathiason-Dubard, G. H. Learn, A. G. Rodrigo, D. L. Sodora, P. Mazzetti, H. A. Hoover, and J. I. Mullins, Genetic diversity of feline immunodeficiency virus: dual infection, recombination, and distinct evolutionary rates among envelope sequence clades, J. Virol. 71, 4241-4253 (1997).

151. E. A. Weaver, E. W. Collisson, M. Slater, and G. Zhu, Phylogenetic analysies of Texas isolates indicate an evolving subtype of the clade B feline immunodeficiency viruses, J. Virol. 78, 2158-2163 (2004).

152. M. T. Kyaw-Tanner, W. K. Greene, H. S. Park, and H. S. Robinson, The induction of in vivo superinfection and recombination using feline immunodeficiency virus as the model, Arch. Virol. 138, 261-271 (1994).

153. F. Reggeti and D. Bienzle, Feline immunodeficiency virus subtypes A, B and C and intersubtype recombinants in Ontario, Canada, J. Gen. Virol. 85, 1843-1852 (2004).

154. E. C. Sabino, E. G. Shpaer, M. G. Morgado, B. T. M. Korber, R. Diaz, V. Bongertz, S. Cavalcante, B. Galvao-Castro, J. I. Mullins, and A. Mayer, Identification of human immunodeficiency virus type 1 envelope genes recombinant between subtypes $\mathrm{B}$ and $\mathrm{F}$ in two epidemiologically linked individuals in Brazil, J. Virol. 68, 6340-6346 (1994).

155. D. L. Robertson, B. H. Hahn, and P. M. Sharp, Recombination in AIDS viruses, J. Mol. Evol. 40, 249-259 (1995).

156. D. L. Robertson, P. M. Sharp, F. E. McCutchan, and B. H. Hahn, Recombination in HIV-1, Nature 37, 124-126 (1995).

157. M. C. Gemeniano, E. T. Sawai, C. M. Leutenegger, and E. E. Sparger, Feline immunodeficiency virus orf-A is required for virus particle formation and virus infectivity, J. Virol. 77, 8819-8830 (2003).

158. M. C. Gemeniano, E. T. Sawai, and E. E. Sparger, Feline immunodeficiency virus Orf-A localizes to the nucleus and induces cell cycle arrest, Virology 253, 167-174 (2004).

159. M. H. Malim, L. S. Tiley, D. F. McCarn, J. R. Rusche, J. Hauber, and B. R. Cullen, HIV1 structural gene expression requires binding of the Rev trans-activator to its RNA target sequence, Cell 60, 675-683 (1990).

160. B. K. Felber, M. Hadzopoulou-Cladaras, C. Cladaras, T. Copeland, and G. N. Pavlakis, Rev protein of human immunodeficiency virus type 1 affects the stability and transport of the viral mRNA, Proc. Natl. Acad. Sci. USA 86, 1494-1499 (1989).

161. G. C. Otero, M. E. Harris, J. E. Donello, and T. J. hope, Leptomycin B inhibits equine infectious anemia virus Rev and feline immunodeficiency virus Rev function but not the function of the hepatitus B virus posttranscriptional regulatory element, J. Virol. 72, $7593-7597$ (1998).

162. V. A. Mancuso, T. J. Hope, L. Zhu, D. Derse, T. Phillips, and T. G. Parslow, Posttranscriptional effector domains in the Rev proteins of feline immunodeficiency virus and equine infectious anemia virus, J. Virol. 68, 1998-2001 (1994).

163. T. J. Hope, Viral RNA export, Chem. Biol. 4, 335-344 (1997).

164. O. Schatz, M. Oft, C. Dascher, M. Schebesta, O. Rosorius, H. Jaksche, M. Dobrovnik, D. Bevec, and J. Hauber, Interaction of the HIV-1 rev cofactor eukaryotic initiation factor 5A with ribosomal protein L5, Proc. Natl. Acad. Sci. USA 95, 1607-1612 (1998).

165. D. Bevec, H. Jaksche, M. Oft, T. Wohl, M. Himmellspach, A. Pacher, M. Schebesta, K. Koettnitz, M. Dobrovnik, R. Csonga, F. Lotspeich, and J. Hauber, Inhibition of HIV-1 replication in lymphocytes by mutants of the Rev cofactor eLF-5A, Science 271, 10829-10833 (1996). 
166. R. A. Hart, J. Billaud, S. J. Choi, and T. R. Phillips, Effects of 1,8-diaminooctane on the FIV Rev regulatory system, Virology 304, 97-104 (2002).

167. H. P. Bogerd, A. Echarri, T. M. Ross, and B. R. Cullen, Inhibition of human immunodeficiency virus Rev and human T-cell leukemia virus Rex function, but not Mason-Pfizer monkey virus constitutive transport element activity, by a mutant human nucleoporin targeted to Crm1, J. Virol. 72, 8627-8635 (1998).

168. M. H. Park, E. C. Wolff, and J. E. Folk, Hypusine: its post-translational formation in eukaryotic initiation factor $5 \mathrm{~A}$ and its potential role in cellular regulation, Biofactors 4 , 95-104 (1993).

169. K. Strebel, D. Daugherty, and K. Clouse, The HIV “A” (sor) gene product is essential for virus infectivity, Nature 328, 728-730 (1987).

170. J. Sodroski, W. C. Goh, and C. Rosen, Replicative and cytopathic potential of HTLVII/LAV with sor gene deletions, Science 231, 412-417 (1986).

171. K. Tomonaga, Y. Inoshima, Y. Ikeda, and T. Mikami, Temporal patterns of feline immunodeficiency virus transcripts in peripheral blood cells during the latent stage of infection, J. Gen. Virol. 76, 2193-2204 (1995).

172. K. Tomonaga, J. Norimine, Y. Shin, M. Fukasawa, T. Miyazawa, A. Adachi, T. Toyosaki, Y. Kawaguchi, C. Kai, and T. Mikami, Identification of a feline immunodeficiency virus gene which is essential for cell-free virus infectivity, J. Virol. 66, 6181-6185 (1992).

173. B. L. Shacklett and P. A. Luciw, Analysis of the VIF gene of feline immunodeficiency virus, Virology 204, 860-867 (1994).

174. K. Lockridge, S. Himathongkham, E. T. Sawai, M. Chien, and E. E. Sparger, The feline immunodeficiency virus vif gene is required for productive infection of feline peripheral blood mononuclear cells and monocyte-derived macrophages, Virology 261, 25-30 (1999).

175. U. Chatterji, C. K. Grant, and J. H. Elder, Feline immunodeficiency virus Vif localizes to the nucleus, J. Virol. 74, 2533-2540 (2001).

176. D. H. Gabuzda, K. Lawrence, E. Langhoff, E. Terwillinger, T. Dorfman, W. A. Haseltine, and J. Sodroski, Role of vif in replication of human immunodeficiency virus type 1 in CD4+ T lymphocytes, J. Virol. 66, 6489-6495 (1992).

177. N. Madani and D. Kabat, An endogenous inhibitor of human immunodeficiency virus in human lymphocytes is overcome by the viral vif protein, J. Virol. 72, 10251-10255 (1998).

178. A. M. Sheehy, N. C. Gaddis, J. D. Choi, and M. H. Malim, Isolation of a human gene that inhibits HIV-1 infection and is suppressed by the viral Vif protein, Nature 418, 646-650 (2002).

179. V. N. Kewal-Ramani and J. M. Coffin, Virology. Weapons of mutational destruction, Science 301, 923-925 (2003).

180. Q. Yu, R. Konig, S. Pillai, K. Chiles, M. Kearney, S. Palmer, D. Richman, J. M. Coffin, and N. R. Landau, Single-strand specificity of APOBEC3G accounts for minus-strand deaminatin of the HIV genome, Nat. Struct. Mol. Biol. 11, 435-442 (2004).

181. S. P. Goff, Death by deamination: a novel host restriction system for HIV-1, Cell 114, 281-283 (2003).

182. R. Mariani, D. Chen, B. Schrofelbauer, F. Navarro, R. Konig, B. Bollman, C. Munk, H. Nymark-McMahon, and N. R. Landau, Species-specific exclusion of APOBEC3G from HIV-1 virions by Vif, Cell 114, 21-31 (2003).

183. M. Marin, K. M. Rose, S. L. Kozak, and D. Kabat, HIV-1 Vif protein binds the editing enzyme APOBEC3G and induces its degradation, Nat. Med. 9, 1398-1403 (2003).

184. A. M. Sheehy, N. C. Gaddis, and M. H. Malim, The antiretroviral enzyme APOBEC3G is degraded by the protesome in response to HIV-1 Vif, Nat. Med. 9, 1404-1407 (2003). 
185. R. M. Norway, P. C. Crawford, C. M. Johnson, and A. Mergia, Thymic lesions in cats infected with a pathogenic molecular clone or an ORF-A/2-deficient molecular clone of feline immunodeficiency virus, J. Virol. 75, 5833-5841 (2001).

186. G. Dean and E. E. Sparger, In vivo tropism of molecular cloned isolates of FIV, in Third International Feline Retrovirus Research Symposim. Fort Collins, CO (1996).

187. M. Pistello, M. Moscardini, P. Mazzetti, F. Bonci, L. Zaccaro, P. Isola, G. Freer, S. Specter, I. D. Matteucc, and M. Bendinelli, Development of feline immunodeficiency virus ORFA (tat) mutants: in vitro and in vivo characterization, Virology 298, 84-95 (2002).

188. K. Tomonaga, T. Miyazawa, J. Sakuragi, T. Mori, A. Adachi, and T. Mikami, The feline immunodeficiency virus ORF-A gene facilitates efficient viral replication in established T-cell lines and peripheral blood lymphocytes, J. Virol. 67, 5889-5895 (1993).

189. S. Villet, B. A. Bouzar, T. Morin, G. Verdier, C. Legras, and Y. Chebloune, Maedi-visna virus and caprine arthiritis encphalitis virus genomes encode a Vpr-like but no Tat protein, J. Virol. 77, 9632-9638 (2003).

190. S. De Rozieres, C. K. Mathiason, M. R. Rolston, U. Chatterji, E. A. Hoover, and J. H. Elder, Characterization of a highly pathogenic molecular clone of feline immunodeficiency virus clade C, J. Virol. 78, 8971-8982 (2004).

191. L. Diehl, C. K. Mathiason-Dubard, L. L. O’Neil, and E. A. Hoover, Plasma viral RNA load predicts disease progression in accelerated feline immunodeficiency virus infection, J. Virol. 70, 2503-2507 (1996).

192. E. E. Sparger, A. M. Beebe, N. Dua, J. Elder, S. Himathongkam, M. Torten, and J. Higgins, Infection of cats with molecularly cloned and biological isolates of the feline immunodeficiency virus, Virology 205, 546-553 (1994).

193. J. C. Woo, G. A. Dean, N. C. Pedersen, and P. F. Moore, Immunopathologic changes in the thymus during the acute stage of experimentally induced feline immunodeficiency virus infection in juvenile cats, J. Virol. 71, 8632-8641 (1997).

194. J. Yang, R. V. English, J. W. Ritchey, M. G. Davidson, T. Wasmoen, J. K. Levy, D. H. Gebhard, M. B. Tompkins, and W. A. F. Tompkins, Molecularly cloned feline immunodeficiency virus NCSU1 YSY3 induces immunodeficiency in specific-pathogen-free cats, J. Virol. 70, 3011-3017 (1996).

195. W. C. Brown, L. Bissey, K. S. Logan, N. C. Pedersen, J. H. Elder, and E. W. Collisson, Feline immunodefiency virus infects both CD4+ and CD8+ T lymphocytes, J. Virol. 65, 3359-3364 (1991).

196. D. Brunner and N. C. Pedersen, Infection of peritoneal macrophages in vitro and in vivo with feline immunodeficiency virus, J. Virol. 63, 5483-5488 (1989).

197. G. A. Dean, S. Himathongkham, and E. E. Sparger, Differential cell tropism of feline immunodeficiency virus molecular clones in vivo, J. Virol. 73, 2596-2603 (1999).

198. S. W. Dow, M. L. Poss, and E. A. Hoover, Feline immunodeficiency virus: a neurotropic lentivirus, J. Acquir. Immune Defic. Syndr. 3, 658-668 (1990).

199. S. W. Dow, C. K. Mathiason, and E. A. Hoover, In vivo monocyte tropism of pathogenic feline immunodeficiency viruses, J. Virol. 73, 6852-6861 (1999).

200. N. Dua, G. Reubel, J. Higgins, and N. C. Pedersen, The primary stage of feline immunodeficiency virus infection, Vet. Immunol. Immunopathol. 43, 337-355 (1994).

201. R. V. English, C. M. Johnson, D. H. Gebhard, and M. B. Tompkins, In vivo lympohcyte tropism of feline immunodeficiency virus, J. Virol. 67, 5175-5186 (1993).

202. A. Hein, J. P. Martin, F. Koehren, A. Bingen, and R. Dorries, In vivo infection of ramified microglia from adult cat central nervous system by feline immunodeficiency virus, Virology 268, 420-429 (2000).

203. A. Kipar, F. S. Boretti, M. M. Meli, K. Failing, M. Reinacher, and H. Lutz, Reduced constitutive cytokine transcription in isolated monocytes of clinically healthy cats, infected with an FIV strain of low pathogenicity, Vet. Immunol. Immunopathol. 98, 215-221 (2004). 
204. M. Magnani, L. Rossi, A. Fraternale, L. Silvotti, F. Quintavalla, G. Piedimonte, D. Matteucci, F. Baldinotti, and M. Bendinelli, Feline immunodeficiency virus infection of

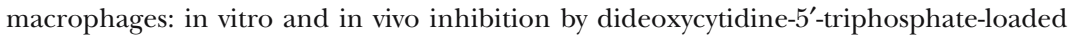
erythrocytes, AIDS Res. Hum. Retroviruses 10, 1179-1186 (1994).

205. K. Nakagaki, K. Nakagaki, K. Takahashi, D. Schols, E. De Clercq, and T. Tabira, CXCR4 is the primary receptor for feline immunodeficiency virus in astrocytes, J. Neurovirol. 7 , 487-492 (2001).

206. A. Rogers and E. Hoover, Fetal feline immunodeficiency virus is prevalent and occult, J. Infect. Dis. 186, 895-904 (2002).

207. A. B. Rogers, C. K. Mathiason, and E. A. Hoover, Immunohistochemical localization of feline immunodeficiency virus using native species antibodies, Am. J. Pathol. 161, 1143-1151 (2002).

208. T. Toyosaki, T. Miyazawa, T. Furuya, K. Tomonaga, Y.-S. Shin, M. Okita, Y. Kawaguchi, C. Kai, S. Mori, and T. Mikami, Localizaion of the viral antigen of feline immunodeficiency virus in the lymph nodes of cats at the early stage of infection, Arch. Virol. 131, 335-347 (1993).

209. T. W. Vahlenkamp, A. De Ronde, N. N. Schuurman, A. L. Van Vliet, J. Van Drunen, M. C. Horzinek, and H. F. Egberink, Envelope gene sequences encoding variable regions 3 and 4 are involved in macrophage tropism of feline immunodeficiency virus, J. Gen. Virol. 80, 2639-2646 (1999).

210. N. Yu, J. N. Billaud, and T. R. Phillips, Effects of feline immunodeficiency virus on astrocyte glutamate uptake: implications for lentivirus-induced central nervous system diseases, Proc. Natl. Acad. Sci. USA 95, 2624-2629 (1998).

211. D. L. Lerner, C. K. Grant, A. P. De Parseval, and J. H. Elder, FIV infection of IL-2dependent and -independent feline lymphocyte lines: host cells range distinctions and specific cytokine upregulation, Vet. Immunol. Immunopathol. 65, 277-297 (1998).

212. M. J. Hosie, B. J. Willett, D. Klein, T. H. Dunsford, C. Cannon, M. Shimojima, J. C. Neil, and $\mathrm{O}$. Jarett, Evolution of replication efficiency following infection with a molecularly cloned feline immunodeficiency virus of low virulence, J. Virol. 76, 6062-6072 (2002).

213. J. Norimine, T. MIyazawa, Y. Kawaguchi, K. Tomonaga, Y. S. Shin, T. Toyosaki, M. Kohmoto, M. Niikura, Y. Tohya, and T. Mikami, Feline CD4 molecules expressed on feline non-lymphoid cell lines are not enough for productive infection of highlt lymphotropic feline immunodeficiency virus isolates, Arch. Virol. 130, 171-178 (1993).

214. B. J. Willet, M. J. Hosie, O. Jarrett, and J. C. Neil, Identification of a putative cellular receptor for feline immunodeficiency virus as the feline homologue of CD9, Immunology 81, 228-233 (1994).

215. M. J. Hosie, B. J. Willett, T. H. Dunsford, O. Jarrett, and J. C. Neil, A monoclonal antibody which blocks infection with feline immunodeficiency virus identifies a possible nonCD4 receptor, J. Virol. 67, 1667-1671 (1993).

216. A. De Parseval, D. L. Lerner, P. Borrow, B. J. Willett, and J. H. Elder, Blocking of feline immunodeficiency virus infection by a monoclonal antibody to CD9 is via inhibition of virus release rather than interference with receptor binding, J. Virol. 71, 5742-5749 (1997).

217. B. J. Willett, M. J. Hosie, J. C. Neil, J. D. Turner, and J. A. Hoxie, Common mechanism of infection by lentiviruses, Nature 385, 587 (1997).

218. B. J. Willett, K. Adema, N. Heveker, A. Brelot, L. Picard, M. Alizon, J. D. Turner, J. A. Hoxie, S. Peiper, J. C. Neil, and M. J. Hosie, The extracellular loop of CXCR4 determines its function as a receptor for feline immunodeficiency virus, J. Virol. 72, 6475-6481 (1998).

219. M. J. Hosie, N. Broere, J. Hesselgesser, J. D. Turner, J. A. Hoxie, J. C. Neil, and B. J. Willett, Modulation of feline immunodeficiency virus infection by stromal cell-derived factor (SDF-1), J. Virol. 72, 2097-2104 (1998). 
220. H. F. Egberink, E. De Clercq, A. L. W. Van Vliet, J. Balzarini, G. J. Bridger, G. Henson, M. C. Horzinek, and D. Schols, Bicyclams, selective antagonists of the human chemokine receptor CXCR4, potently inhibit feline immunodeficiency virus replication, J. Virol. 73, 6346-6352 (1999).

221. J. Richardson, G. Pancino, R. Merat, T. Leste-Lasserre, A. Maraillon, J. SchneiderMergener, M. Alizon, P. Sonigo, and N. Heveker, Shared usage of the chemokine receptor CXCR4 by primary and laboratory-adapted strains of feline immunodeficiency virus, J. Virol. 73, 3661-3671 (1999).

222. A. Brelot, N. Heveker, K. Adema, M. J. Hosie, B. Willett, and M. Alizon, Effect of mutations in the second extracellular loop of CXCR4 on its utilization by human and feline immunodeficiency viruses, J. Virol. 73, 2576-2586 (1999).

223. J. C. Johnston and C. Power, Feline immunodeficiency virus xenoinfection: the role of chemokine receptors and envelope diversity, J. Virol. 76, 3626-3636 (2002).

224. B. J. Willett, C. A. Cannon, and M. J. Hosie, Upregulation of surface feline CXCR4 expression following ectopic expression of CCR5: implications for studies of the cell tropism of feline immunodeficiency virus, J. Virol. 76, 9242-9252 (2002).

225. J. C. Johnston, M. Gasmi, L. E. Lim, J. H. Elder, J. Yee, D. J. Jolly, K. P. Campbell, B. L. Davison, and S. L. Sauter, Minimum requirements for efficient transduction of dividing and nondividing cells by feline immunodeficiency virus vectors, J. Virol. 73, 4991-5000 (1999).

226. A. De Parseval, S. Ngo, P. Sun, and J. H. Elder, Factors that increase the effective concentration of CXCR4 dictate feline immunodeficiency virus tropism and kinetics of replication, J. Virol. 78, 9132-9143 (2004).

227. A. De Parseval, U. Chatterji, P. Sun, and J. H. Elder, Feline immunodeficiency virus targets activated CD4+ T-cells by using CD134 as a binding receptor, Proc. Natl. Acad. Sci. USA 101, 13044-13049 (2004).

228. A. D. Weinberg, D. E. Evans, C. Thalhofer, T. Shi, and R. A. Prell, The generation of Tcell memory: a review describing the molecular and cellular events following OX40 (CD1334) engagement, J. Leukocyte Biol. 75, 1-11 (2004).

229. P. R. Baum, R. B. I. Gayle, F. Ramsdell, S. Srinivasan, R. A. Sorensen, M. L. Watson, M. F. Seldin, E. Baker, G. R. Sutherland, K. N. Clifford, et al., Molecular characterization of murine and human OX40/OX40 ligand systems: identification of a human OX40 ligand as the HTLV-1-regulated protein gp34, EMBO J. 13, 3992-4001 (1994).

230. H. Durkop, I. Anagnostopoulos, S. Bulfone-Paus, and H. Stein, Expression of several members of the TNF-ligand and receptor family on tonsillar lymphoid B cells, Br. J. Haematol. 98, 863-868 (1997).

231. H. Durkop, U. Latza, P. Himmelreich, and H. Stein, Expression of the human OX40 (hOX40) antigen in normal and neoplastic tissues, Br. J. Haematol. 91, 927-931 (1995).

232. J. D. Siliciano, J. Kajdas, D. Finzi, T. C. Quinn, K. Chadwick, J. B. Margolick, C. Kovacs, S. J. Gange, and R. F. Siliciano, Long-term follow-up studies confirm the stability of the latent reservoir for HIV-1 in resting CD4+ T-cells, Nat. Med. 9, 727-728 (2003).

233. R. S. Veazey, I. C. Tham, K. G. Mansfield, M. DeMaria, A. E. Forand, D. E. Shvetz, L. V. Chalifoux, P. K. Sehgal, and A. A. Lackner, Identifying the target cell in primary simian immunodeficiency virus (SIV) infection: highly activated memory CD4(+) T-cells are rapidly eliminated in early SIV infection in vivo, J. Virol. 74, 57-64 (2000).

234. Z. Q. Zhang, S. W. Wietgrefe, Q. Li, M. D. Shore, L. Duan, C. Reilly, J. D. Lifson, and A. T. Haase, Roles of substrate availability and infection of resting and activated CD4+ T-cells in transmission and acute simian immunodeficiency virus infection, Proc. Natl. Acad. Sci. USA 101, 5640-5645 (2004).

235. Z. Zhang, T. Schuler, M. Zupancic, S. Wietgrefe, et al., Sexual transmission and propagation of SIV and HIV in resting and activated CD4(+) T-cells, Science 286, 1353-1357 (1999). 
236. D. M. Willerford, M. J. Gale, R. E. Benveniste, E. A. Clark, and W. M. Gallatin, Simian immunodeficiency virus is restricted to a subset of blood CD4+ lymphocytes that includes memory cells, J. Immunol. 144, 3779-3783 (1990).

237. S. M. Schnittman, H. C. Lane, J. J. Greenhouse, J. S. Justement, M. Baseler, and A. S. Fauci, Preferential infection of CD4+ memory T-cells by human immunodeficiency virus type 1: evidence for a role in the selective T-cell functional defects observed in infected individuals, Proc. Natl. Acad. Sci. USA 87, 6058-6062 (1990).

238. T. Hohdatsu, H. Hirabayashi, K. Motokawa, and H. Koyama, Comparative study of the cell tropism of feline immunodeficiency virus isolates of subtypes A, B and D classified on the basis of the env gene V3-V5 sequence, J. Gen. Virol. 77, 93-100 (1996).

239. T. Miyazawa, T. Furuya, S. Itagaki, Y. Tohya, K. Nakano, E. Takahashi, and T. Mikami, Preliminary comparisons of the biological properties of two strains of feline immunodeficiency virus (FIV) isolated in Japan with FIV Petaluma strain isolated in the United States, Arch. Virol. 108, 59-68 (1989).

240. R. A. Olmsted, A. K. Barnes, J. K. Yamamoto, V. M. Hirsch, R. H. Purcell, and P. R. Johnson, Molecular cloning of feline immunodeficiency virus, Proc. Natl. Acad. Sci. USA 86, 2448-2452 (1989).

241. B. J. Willett, M. J. Hosie, T. H. Dunsford, J. C. Neil, and O. Jarrett, Productive infection of T-helper lymphocytes with feline immunodeficiency virus is accompanied by reduced expression of CD4, AIDS 5, 1469-1475 (1991).

242. J. K. Yamamoto, C. D. Ackley, H. Zochlinski, H. Louie, E. Pembroke, M. Torten, H. Hansen, R. Munn, and T. Okuda, Development of IL-2-independent feline lymphoid cell lines chronically infected with feline immunodeficiency virus: importance for diagnostic reagents and vaccines, Intervirology 32, 361-375 (1991).

243. K. H. Siebelink, J. A. Karlas, G. F. Rimmelzwaan, A. D. Osterhaus, and M. L. Bosch, A determinant of feline immunodeficiency virus involved in Crandell feline kidney cell tropism, Vet. Immunol. Immunopathol. 46, 61-69 (1995).

244. E. J. Verschoor, L. A. Boven, H. llaak, A. L. W. Van Vliet, M. C. Horzinek, and A. De Ronde, A single mutation within the V3 envelope neutralization domain of feline immunodeficiency virus determines its tropism for CRFK cells, J. Virol. 69, 4752-4757 (1995).

245. D. L. Lerner and J. H. Elder, Expanded host cell tropism and cytopathic properties of feline immunodeficiency virus strain PPR subsequent to passage through interleukin-2independent T-cells, J. Virol. 74, 1854-1863 (2000).

246. M. Kohmoto, T. Miyazawa, K. Tomonaga, Y. Kawaguchi, T. Mori, Y. Tohya, C. Kai, and T. Mikami, Comparison of biological properties of feline immunodeficiency virus isolates using recombinant chimeric viruses, J. Gen. Virol. 75, 1935-1942 (1994).

247. T. W. Vahlenkamp, E. J. Verschoor, N. N. Schuurman, A. L. Van Vliet, M. C. Horzinek, H. F. Egberink, and A. De Ronde, A single amino acid substitution in the transmembrane envelope glycoprotein of feline immunodeficiency virus alters cellular tropism, J. Virol. 71, 7132-7135 (1997).

248. G. Pancino, S. Castelot, and P. Sonigo, Differences in feline immunodeficiency virus host cell range correlate with envelope fusogenic properties, Virology 206, 796-806 (1995).

249. T. Shioda, J. A. Levy, and C. Cheng-Mayer, Small amino acid changes in the V3 hypervariable region of gp120 can affect the T-cell line and macrophage tropism of human immunodeficiency virus type 1, Proc. Natl. Acad. Sci. USA 89, 9434-9438 (1992).

250. T. Shioda, J. A. Levy, and C. Cheng-Mayer, Macrophage and T-cell-line tropisms of HIV1 are determined by specific regions of the envelope gp120 gene, Nature 349, 167-169 (1991).

251. J. J. De Jong, A. De Ronde, W. Keulen, M. Tersmette, and J. Goudsmit, Minimal requirements for the human immunodeficiency virus type $1 \mathrm{~V} 3$ domain to support the 
syncytium-inducing phenotype: analysis by single amino acid substitution, J. Virol. 66, 6777-6780 (1992).

252. A. De Ronde, J. G. Stam, P. Boers, H. Langedijk, R. Meloen, W. Hesselink, L. C. Keldermans, A. Van Vliet, E. J. Verschoor, M. C. Horzinek, and H. F. Egberink, Antibody response in cats to the envelope proteins of feline immunodeficiency virus: identification of an immunodominant neutralization domain, Virology 198, 257-264 (1994).

253. S. Lombardi, C. Garzelli, M. Pistello, C. Massi, D. Matteucci, F. Baldinotti, G. Cammarota, L. Da Prato, P. Bandecchi, F. Tozzini, and M. Bendinelli, A neutralizing antibodyinducing peptide of the V3 domain of feline immunodeficiency virus envelope glycoprotein does not induce protective immunity, J. Virol. 68, 8374-8379 (1994).

254. S. Lombardi, C. Massi, F. Tozzini, L. Zaccaro, P. Bancecchi, C. La Rosa, M. Bendinelli, and C. Garzelli, Epitope mapping of the V3 domain of feline immunodeficiency virus envelope glycoprotein by monoclonal antibodies, J. Gen. Virol. 76, 1893-1899 (1995).

255. A. Hein, H. Schuh, S. Thiel, J. P. Martin, and R. Dorries, Ramified feline microglia selects for distinct variants of feline immunodeficiency virus during early central nervous system infection, J. Neurovirol. 9, 465-476 (2003).

256. T. Miyazawa, Y. Kawaguchi, M. Kohmoto, K. Tomonaga, and T. Mikami, Comparative functional analysis of the various lentivirus long terminal repeats in human colon carcinoma cell line (SW489 cells) and feline renal cell line (CRFK cells), J. Vet. Med. Sci. 56, 895-899 (1994).

257. T. Miyazawa, Y. Kawaguchi, M. Kohmoto, J. Sakuragi, A. Adachi, M. Fukasawa, and T. Mikami, Production of feline immunodeficiency virus in feline and non-feline nonlymphoid cell lines by transfection of an infectious molecular clone, J. Gen. Virol. 73, 1543-1546 (1992).

258. Y. Ikeda, K. Tomonaga, Y. Kawaguchi, M. Kohmoto, Y. Inoshima, Y. Tohya, T. Miyazawa, C. Kai, and T. Mikami, Feline immunodeficiency virus can infect a human cell line (MOLT-4) but establishes a state of latency in the cells, J. Gen. Virol. 77, 1623-1630 (1996).

259. T. S. Tochikura, A. Tanabe-Tochikura, K. Hayes, A. Lazo, R. T. Bailer, J. R. Blakeeslee, L. J. Lafrado, P. Roy-Burman, R. Pandey, R. G. Olsen, and L. E. Mathes, Fusion activity dissociated from replication ability in feline immunodeficiency virus (FIV) in human cells, JAIDS 6, 1297-1300 (1993).

260. E. M. Poeschla, F. Wong-Staal, and D. J. Looney, Efficient transduction of nondividing human cells by feline immunodeficiency virus lentiviral vectors, Nature Med. 4, 354-357 (1998).

261. J. Johnston and C. Power, Productive infection of human peripheral blood mononuclear cells by feline immunodeficiency virus: implications for vector development, J. Virol. 73, 2491-2498 (1999).

262. J. B. Johnston, M. E. Olson, E. W. Rud, and C. Power, Xenoinfection of nonhuman primates by feline immunodeficiency virus, Curr. Biol. 11, 1109-1113 (2001).

263. M. C. Barr, M. B. Pough, R. H. Jacobson, and F. W. Scott, Comparison and interpretation of diagnostic tests for feline immunodeficiency virus infection, J. Am. Vet. Med. Assoc. 199, 1377-1381 (1991).

264. R. Groat, G. Maddsen, B. Bartol, P. Skvorak, K. Serlemitsos, and M. Monn. Upgraded IDEXX diagnostic products for simultaneous detection of antibodies to feline immunodeficiency virus (FIV) gag and env proteins in feline blood samples. in 25th World Small Animal Veterinary Association / 6th Federation of European Companion Animal Veterinary Association / Voorjaarsdagen Congress. Amsterdam, The Netherlands (2000).

265. K. Hartmann, R. M. Werner, H. Egberink, and O. Jarrett, Comparison of six in-house tests for the rapid diagnosis of feline immunodeficiency and feline leukemia virus infections, Vet. Rec. 149, 317-320 (2001). 
266. T. P. O'Connor, Q. J. Tonelli, and J. M. Scarlett, Report of the national FeLV/FIV awareness project, J. Am. Vet. Med. Assoc. 199, 1348-1353 (1991).

267. J. D. Fontenot, E. A. Hoover, J. H. Elder, and R. C. Montelaro, Evaluation of feline immunodeficiency virus and feline leukemia virus transmembrane peptides for serological diagnosis, J. Clin. Microbiol. 30, 1885-1890 (1992).

268. M. J. Hosie, C. Robertson, and O. Jarrett, Prevalence of feline leukemia virus and antibodies to feline immunodeficiency virus in cats in the United Kingdom, Vet. Rec. 125, 293-297 (1989).

269. R. A. Olmsted, R. Langley, M. E. Roelke, R. M. Goeken, D. Adger-Johnson, J. P. Goff, J. P. Alber, C. Packer, M. K. Laurenson, T. M. Caro, L. Scheepers, D. E. Wildt, M. Bush, J. S. Martenson, and S. J. O'Brien, Worldwide prevalence of lentivirus infection in wild felidae species: epidemiologic and phylogenetic aspects, J. Virol. 66, 6008-6018 (1992).

270. I. Lee, J. A. Levy, S. Gorman, P. C. Crawford, and M. Slater, Prevalence of feline leukemia virus infection and serum antibodies against feline immunodeficiency virus in unowned free-roaming cats, J. Am. Vet. Med. Assoc. 220, 620-622 (2002).

271. M. Uema, Y. Ikeda, and T. Miyazawa, Feline immunodeficiency virus subtype C is prevalent in northern part of Taiwan, J. Vet. Med. Sci. 61, 197-199 (1999).

272. I. G. Winkler, M. Lochelt, and R. L. P. Flower, Epidemiology of feline foamy virus and feline immunodeiciency virus infections in domestic and feral cats: a seroepidemiological study, J. Clin. Microbiol. 37, 2848-2851 (1999).

273. F. Courchamp and D. Pontier, Feline immunodeficiency virus: an epidemiological review, C. R. Acad. Sci. III 317, 1123-1134 (1994).

274. E. Sparger, P. Luciw, J. Elder, J. Yamamoto, L. Lowenstine, and N. Pedersen, Feline immunodeficiency virus is a lentivirus associated with an AIDS-like disease in cats, AIDS 3(Suppl 1), S43-49 (1989).

275. M. C. Barr, L. Zou, D. L. Holzschu, L. Pillips, F. W. Scott, J. W. Casey, and R. J. Avery, Isolation of a highly cytopathic lentivirus from a nondomestic cat, J. Virol. 69, 7371-7374 (1995).

276. E. W. Brown, S. Miththapala, and S. J. O'Brien, Prevalence of exposure to feline immunodeficiency virus in exotic felid species, J. Zoo. Wildl. Med. 24, 357-364 (1993).

277. E. W. Brown, N. Yuhki, C. Packer, and S. J. O'Brien, A lion lentivirus related to feline immunodeficiency virus: epidemiologic and phylogenetic aspects, J. Virol. 68, 5953-5968 (1994).

278. H. Lutz, E. Isenbugel, R. Lehmann, R. Sabapara, and C. Wolfensberger, Retrovirus infections in non-domestic felids: serological studies and attempts to isolate a lentivirus, Vet. Immunol. Immunopathol. 35, 215-224 (1992).

279. Y. Nishimura, Y. Goto, K. Yoneda, Y. Endo, T. Mizuno, M. Hamachi, H. Maruyama, H. Kinoshita, S. Koga, M. Komori, S. Fushuku, K. Ushinohama, M. Akuzawa, T. Watari, A. Hasegawa, and H. Tsujimoto, Interspecies transmission of feline immunodeficiency virus from the domestic cat to the Tsushima cat, J. Virol. 73, 7916-7921 (1999).

280. J. A. Spencer, A. A. Van Dijk, M. C. Horzinek, H. F. Egberink, R. G. Bengis, D. F. Keet, S. Morikawa, and D. H. L. Bishop, Incidence of feline immunodeficiency virus reactive antibodies in free-ranging lions of the Kruger National Park and the Etosha National Park in southern Africa detected by recombinant FIV p24 antigen, Onderstepoort J. Vet. Res. 59, 315-322 (1992).

281. R. Hofmann-Lehmann, D. Fehr, M. Grob, M. Elgizoli, C. Packer, J. S. Martenson, S. J. O'Brien, and H. Lutz, Prevalence of antibodies to feline parvovirus, calicivirus, herpesvirus, coronavirus, and immunodeficiency virus and of feline leukemia virus antigen and the interrelationship of these viral infections in free-ranging lions in East Africa, Clin. Diagn. Lab. Immunol. 3, 554-562 (1996). 
282. R. Biek, A. G. Rodrigo, D. Holley, A. Drummond, C. R. Anderson, H. A. Ross, and M. Poss, Epidemiology, genetic diversity, and evolution of endemic feline immunodeficiency virus in a population of wild cougars, J. Virol. 77, 9578-9589 (2003).

283. F. Courchamp, D. Pontier, M. Langlais, and M. Artois, Population dynamics of feline immunodeficiency virus within cat populations, J. Theor. Biol. 175, 553-560 (1995).

284. F. Courchamp, N. G. Yoccoz, M. Artois, and D. Pontier, At-risk individuals in feline immunodeficiency virus epidemiology: evidence from a multivariate approach in a natural population of domestic cats (Felis catus), Epidemiol. Infect. 121, 227-236 (1998).

285. P. Dorny, N. Speybroeck, S. Verstraete, M. Baeke, A. De Becker, D. Berkvens, and J. Vercruysse, Serological survey of Toxoplasma gondii, feline immunodeficiency virus and feline leukemia virus in urban stray cats in Belgium, Vet. Rec. 151, 626-629 (2002).

286. E. Holznagel, H. Lutz, D. Steinhauer, and M. Reinacher, Feline immunodeficiency virus (FIV) infection in cats at necropsy: a serological study, J. Comp. Pathol. 116, 339-352 (1997).

287. B. J. Luria, J. K. Levy, M. R. Lappin, E. B. Breitschwerdt, A. M. Legendre, J. A. Hernandez, S. P. Gorman, and I. T. Lee, Prevalence of infectious diseases in feral cats in Northern Florida, J. Feline Med. Surg. 6, 287-296 (2004).

288. N. C. Pedersen, in The Retroviridae Vol. 2, edited by J. A. Levy (Plenum Press, New York, 1993), 181-227

289. D. Pontier, E. Fromont, F. Courchamp, M. Artois, and N. G. Yoccoz, Retroviruses and sexual size dimorphism in domestic cats (Felis catus L.), Proc. R. Soc. Lond. B. Biol. Sci. 265, 167-173 (1998).

290. D. Matteucci, F. Baldinotti, P. Mazzetti, M. Pistello, P. Bandecchi, R. Ghilarducci, A. Poli, F. Tozzini, and M. Bendinelli, Detection of feline immunodeficiency virus in saliva and plasma by cultivation and polymerase chain reaction, J. Clin. Microbiol. 31, 494-501 (1993).

291. K. Ueland and L. Nesse, No evidence of vertical transmission of naturally acquired feline immunodeficiency virus infection, Vet. Immunol. Immunopathol. 33, 301-308 (1992).

292. J. J. Callanan, M. J. Hosie, and O. Jarrett, Transmission of feline immunodeficiency virus from mother to kitten, Vet. Rec. 128, 332-333 (1991).

293. R. K. Sellon, H. L. Jordan, S. Kennedy-Stoskopf, M. B. Tompkins, and W. A. Tompkins, Feline immunodeficiency virus can be experimentally transmitted via milk during acute maternal infection, J. Virol. 68, 3380-3385 (1994).

294. A. B. Rogers and E. A. Hoover, Maternal-fetal feline immunodeficiency virus transmission: timing and tissue tropisms, J. Infect. Dis. 178, 960-967 (1998).

295. R. Allison and E. Hoover, Feline immunodeficiency virus is concentrated in milk early in lactation, AIDS Res. Hum. Retroviruses 19, 245-253 (2003).

296. L. L. O'Neil, M. J. Burkhard, L. A. Obert, and E. A. Hoover, Regression of feline immunodeficiency virus infection, AIDS Res. Hum. Retroviruses 13, 713-718 (1997).

297. R. Allison and E. Hoover, Covert vertical transmission of feline immunodeficiency virus, AIDS Res. Hum. Retroviruses 19, 421-434 (2003).

298. S. Dandekar, A. M. Beebe, J. Barlough, T. Phillips, J. Elder, M. Torten, and N. Pedersen, Detection of feline immunodeficience virus (FIV) nucleic acids in FIV-seronegative cats, J. Virol. 66, 4040-4049 (1992).

299. C. J. Miller, M. Marthas, J. Torten, N. J. Alexander, J. P. Moore, G. F. Doncel, and A. G. Hendrickx, Intravaginal inoculation of rhesus macaques with cell-free simian immunodeficiency virus results in persistent or transient viremia, J. Virol. 68, 6391-6400 (1994).

300. M. B. McChesney, J. R. Collins, D. Lu, X. Lü, J. Torten, R. L. Ashley, M. W. Cloyd, and C. J. Miller, Occult systemic infection and persistent SIV-specific CD4+ T-cell proliferative responses in rhesus macaques that were transiently viremic after intravaginal inoculation of SIV, J. Virol. 72, 10029-10035 (1998). 
301. P. Palumbo, J. Skurnick, D. Lewis, and M. Eisenberg, PCR analysis of HIV-seronegative, heterosexual partners of HIV-infected individuals, J. Acquir. Immune Defic. Syndr. Hum. Retrovirol. 10, 436-440 (1995).

302. G. K. Sahu, J. J. Chen, J. C. Huang, K. M. Ramsey, and M. W. Cloyd, Transient or occult HIV-1 infection in high-risk adults, AIDS 15, 1175-1177 (2001).

303. H. L. Jordan, J. Howard, M. C. Barr, S. Kennedy-Stoskopf, J. K. Levy, and W. A. Tompkins, Feline immunodeficiency virus is shed in semen from experimentally and naturally infected cats, AIDS Res. Hum. Retroviruses 14, 1087-1092 (1998).

304. H. L. Jordan, Y. Liang, L. C. Hudson, and W. A. Tompkins, Shedding of feline immunodeficiency virus in semen of domestic cats during acute infection, Am. J. Vet. Res. 60, 211-215 (1999).

305. H. L. Jordan, J. Howard, R. K. Sellon, D. E. Wildt, W. A. Tompkins, and S. KennedyStoskopf, Transmission of feline immunodeficiency virus in domestic cats via artificial insemination, J. Virol. 70, 8224-8228 (1996).

306. H. L. Jordan, J. G. Howard, J. G. Bucci, J. L. Butterworth, R. V. English, S. KennedyStoskopf, M. B. Tompkins, and W. A. Tompkins, Horizontal transmission of feline immunodeficiency virus with semen from seropositive cats, J. Reprod. Immunol. 41, 341-357 (1998).

307. R. J. Avery and E. A. Hoover, Gamma interferon/interleukin 10 balance in tissue lymphocytes correlates with down modulation of mucosal feline immunodeficiency virus infection, J. Virol. 78, 4011-4019 (2004).

308. J. G. Bucci, R. V. English, H. L. Jordan, T. A. Childers, M. B. Tompkins, and W. A. Tompkins, Mucosally transmitted feline immunodeficiency virus induces a CD8+ antiviral response that correlates with reduction of cell-associated virus, J. Infect. Dis. 177, 18-25 (1998).

309. M. J. Burkhard, C. K. Mathiason, K. O’Halloran, and E. A. Hoover, Kinetics of early FIV infection in cats exposed via the vaginal versus intravenous route, AIDS Res. Hum. Retroviruses 18, 217-226 (2002).

310. T. R. Moench, K. J. Whaley, T. D. Mandrell, B. D. Bishop, C. J. Witt, and R. A. Cone, The cat/feline immunodeficiency virus animal model for transmucosal transmission of AIDS: nonoxynol-9 contraceptive jelly blocks transmission by an infected cell inoculum, AIDS 7, 797-802 (1993).

311. M. Shimojima, Y. Nishimura, T. Miyazawa, Y. Tohya, and H. Akashi, T-cell subpopulations mediating inhibition of feline immunodeficiency virus replication in mucosally infected cats, Microbes Infect. 6, 265-271 (2004).

312. T. Miyazawa, T. Furuya, S. Itagaki, Y. Tohya, E. Takahashi, and T. Mikami, Establishment of a feline T-lymphoblastoid cell line highly sensitive for replication of feline immunodeficiency virus, Arch. Virol. 108, 131-135 (1989).

313. Y. Goto, Y. Nishimura, K. Baba, T. Mizuno, Y. Endo, K. Masuda, K. Ohno, and H. Tsujimoto, Association of plasma viral RNA load with prognosis in cats naturally infected with feline immunodeficiency virus, J. Virol. 76, 10079-10083 (2002).

314. S. T. Dunham, J. N. Flynn, M. A. Rigby, J. MacDonald, J. Bruce, C. Cannon, M. C. Golder, L. Hanlon, D. A. Harbour, N. A. Mackay, N. Spibey, O. Jarrett, and J. C. Neil, Protection against feline immunodeficiency virus using replication defective proviral DNA vaccines with feline interleukin-12 and -18, Vaccine 20, 1483-1496 (2002).

315. C. M. Leutenegger, D. Klein, R. Hofmann-Lehmann, C. Mislin, U. Hummel, J. Boni, F. Boretti, W. H. Guenzbug, and H. Lutz, Rapid feline immunodeficiency virus provirus quantitation by polymerase chain reaction using the TaqMan ${ }^{\circledR}$ fluorogenic real-time detection system, J. Virol. Methods 78, 105-116 (1999).

316. M. Pistello, D. Matteucci, F. Bonci, P. Isola, P. Mazzetti, L. Zaccaro, A. Merico, D. Del Mauro, N. Flynn, and M. Bendinelli, AIDS vaccination studies using an ex vivo feline immunodeficiency virus model: protection from an intraclade challenge admin- 
istered systemically or mucosally by an attenuated vaccine, J. Virol. 77, 10740-10750 (2003).

317. K. Tokunaga, Y. Nishino, H. Oikawa, C. Ishihara, T. Mikami, and K. Ikuta, Altered cell tropism and cytopathicity of feline immunodeficiency viruses in two different feline CD4positive, CD8-negative cell lines, J. Virol. 66, 3893-3898 (1992).

318. A. Moraillon, F. Barre-Sinoussi, A. Parodi, R. Moraillon, and C. Dauguet, In vitro properties and experimental pathogenic effect of three strains of feline immunodeficiency viruses (FIV) isolated from cats with terminal disease, Vet. Microbiol. 31, 41-54 (1992).

319. A. T. Haase, K. Henry, M. Zupancic, G. Sedgewick, R. A. Faust, H. Melroe, W. Cavert, K. Gebhard, K. A. Staskus, Z. Q. Zhang, P. J. Dailey, H. H. Balfour, A. Erice, and A. S. Perelson, Quantitative image analysis of HIV-1 infection in lymphoid tissue, Science 274, 985-989 (1996).

320. G. Pantaleo, C. Graziosi, L. Butini, P. A. Pizzo, S. M. Schnittman, D. P. Kotler, and A. S. Fauci, Lymphoid organs function as major reservoirs for human immunodeficiency virus, Proc. Natl. Acad. Sci. USA 88, 9838-9842 (1991).

321. T. A. Reinhart, M. J. Rogan, D. Huddleston, D. M. Rausch, L. E. Eiden, and A. T. Haase, Simian immunodeficiency virus burden in tissues and cellular compartments during clinical latency and AIDS, J. Infect. Dis. 176, 1198-1208 (1997).

322. S. Matsumura, T. Ishida, T. Washizu, I. Tomoda, S. Nagata, J. Chiba, and T. Kurata, Pathologic features of acquired immunodeficiency-like syndrome in cats experimentally infected with feline immunodeficiency virus, J. Vet. Med. Sci. 55, 387-394 (1993).

323. M. L. Linenberger, A. M. Beebe, N. C. Pedersen, J. L. Abkowitz, and S. Dandekar, Marrow accessory cell infection and alterations in hematopoiesis accompany severe neutropenia during experimental acute infection with feline immunodeficiency virus, Blood 85, 941-951 (1995).

324. M. Podell, M. Oglesbee, L. Mathes, S. Krakowka, R. Olmstead, and L. Lafrado, AIDSassociated encephalopathy with experimental feline immunodeficiency virus infection, J. Acquir. Immune Defic. Syndr. 6, 758-771 (1993).

325. C. R. Stokes, S. Finerty, T. J. Gruffydd-Jones, C. P. Sturgess, and D. A. Harbour, Mucosal infection and vaccination against feline immunodeficiency virus, J. Biotechnol. 73, 213-221 (1999).

326. G. Ryan, D. Klein, E. Knapp, M. J. Hosie, T. Grimes, M. J. Mabruk, O. Jarrett, and J. J. Callanan, Dynamics of viral and proviral loads of feline immunodeficiency virus within the feline central nervous system during the acute phase following intravenous infection, J. Virol. 77, 7477-7485 (2003).

327. T. Tanabe and J. K. Yamamoto, Phenotypic and functional characteristics of FIV infection in the bone marrow stroma, Virology 282, 113-122 (2001).

328. J. C. Woo, G. A. Dean, A. Lavoy, R. Clark, and P. F. Moore, Investigation of recombinant human insulin-like growth factor type $\mathrm{I}$ in thymus regeneration in the acute stage of experimental FIV infection in juvenile cats, AIDS Res. Hum. Retroviruses 15, 1377-1388 (1999).

329. J. B. Johnston, C. Silva, and C. Power, Envelope gene-mediated neurovirulence in feline immunodeficiency virus infection: induction of matrix metalloproteinases and neuronal injury, J. Virol. 76, 2622-2633 (2002).

330. J. B. Johnston, C. Silva, T. Hiebert, R. Buist, M. R. Dawood, J. Peeling, and C. Power, Neurovirulence depends on virus input titer in brain in feline immunodeficiency virus infection: Evidence for activation of innate immunity and neuronal injury, J. Neurovirol. 8, 420-431 (2002).

331. K. Lockridge, M. Chien, P. A. Luciw, and E. E. Sparger, Protective immunity against feline immunodeficiency virus inducedby inoculation with vif-deleted proviral DNA, Virology. 273, 67-79 (2000). 
332. A. J. Phipps, K. A. Hayes, W. R. Buck, M. Podell, and L. E. Mathes, Neurophysiologic and immunologic abnormalities associated with feline immunodeficiency virus molecular clone FIV-PPR DNA inoculation, J. Acquir. Immune Defic. Syndr. 23, 8-16 (2000).

333. C. J. Miller, D. W. Kang, M. Marthas, Z. Moldoveanu, H. Kiyono, P. Marx, J. H. Eldridge, J. Mestecky, and J. R. McGhee, Genital secretory immune response to chronic simian immunodeficiency virus (SIV) infection: a comparison between intravenously and genitally inoculated rhesus macaques, Clin. Exp. Immunol. 88, 520-526 (1992).

334. J. Bucci, D. Gebhard, T. A. Childers, E. R., M. B. Tompkins, and W. A. Tompkins, The CD8+ phenotype mediating antiviral activity in feline immunodeficiency virus-infected cats is characterized by reduced surface expression of the CD8 $\beta$ chain, J. Infect. Dis. 178, 968-977 (1998).

335. S. W. Dow, M. J. Dreitz, and E. A. Hoove, Feline immunodeficiency virus neurotropism: evidence that astrocytes and microglia are the primary target cells, Vet. Immunol. Immunopathol. 35, 23-35 (1992).

336. A. Hein, J. P. Martin, and R. Dorries, In vitro activation of feline immunodeficiency virus in ramified microglial cells from asymptomatically infected cats, J. Virol. 75, 8090-8095 (2001).

337. E. Zenger, E. Tiffany-Castiglioni, and E. W. Collisson, Cellular mechanisms of feline immunodeficiency virus (FIV)-induced neuropathogenesis, Front. Biosci. 2, 527-537 (1997).

338. M. K. Patrick, J. B. Johnsto, and C. Power, Lentiviral neuropathogenesis: comparative neuroinvasion, neurotropism, neurovirulence, and host neurosusceptibility, J. Virol. 76, 7923-7931 (2002).

339. A. Poli, F. Abramo, C. Di Iorio, C. Cantile, M. Carli, C. Pollera, L. Vago, A. Tosoni, and G. Costanzi, Neuropathology in cats experimentally infected with feline immunodeficiency virus: a morphological, immunocytochemical and morphometric study, J. Neurovirol. 3, 361-368 (1997).

340. A. Poli, M. Pistello, M. A. Carli, F. Abramo, G. Mancuso, E. Nicoletti, and M. Bendinelli, Tumor necrosis factor-alpha and virus expression in the central nervous system of cats infected with feline immunodeficiency virus, J. Neurovirol. 5, 465-473 (1999).

341. T. R. Phillips, O. Prospero-Garcia, D. W. Wheeler, P. C. Wagaman, D. L. Lerner, H. S. Fox, L. R. Whalen, F. E. Bloom, J. H. Elder, and S. J. Henriksen, Neurologic dysfunctions caused by a molecular clone of feline immunodeficiency virus, FIV-PPR, J. Neurovirol. 2, 388-396 (1996).

342. A. A. Lackner, S. Dandekar, and M. B. Gardner, Neurobiology of simian and feline immunodeficiency virus infections, Brain Pathol. 1, 201-212 (1991).

343. S. Huitron-Resendiz, S. De Rozieres, M. Sanchez-Alavez, B. Buhler, Y. C. Lin, D. L. Lerner, N. W. Henriksen, M. Burudi, H. S. Fox, B. E. Torbett, S. J. Henriksen, and J. H. Elder, Resolution and prevention of feline immunodeficiency virus-induced neurological deficits by treatment with the protease inhibitor TL-3, J. Virol. 78, 4525-4532 (2004).

344. M. Hurtrel, J. P. Ganiere, J. F. Guelfi, L. Chakrabarti, M. A. Maire, F. Gray, L. Montagnier, and B. Hurtrel, Comparison of early and late feline immunodeficiency virus encephalopathies, AIDS 6, 399-406 (1992).

345. A. T. Haase, Population biology of HIV-1 infection: viral and CD4+ T-cell demographics and dynamics in lymphatic tissues, Annu. Rev. Immunol. 17, 635-656 (1999).

346. R. S. Veazey, M. DeMaria, L. V. Chalifoux, D. E. Shvetz, D. R. Pauley, H. L. Knight, M. Rosenzweig, R. P. Johnson, R. C. Desrosiers, and A. A. Lackner, Gastrointestinal tract as a major site of CD4+ T-cell depletion and viral replication in SIV infection, Science 280, 427-431 (1998).

347. I. M. Belyakov, Z. Hel, B. Kelsall, V. A. Kuznetsov, J. D. Ahlers, J. Nacsa, D. I. Watkins, T. M. Allen, A. Sette, J. Altman, R. Woodward, P. D. Markham, J. D. Clements, G. Franchini, W. Strober, and J. A. Berzofsky, Mucosal AIDS vaccine reduces disease and 
viral load in gut reservoir and blood after mucosal infection of macaques, Nat. Med. 7 , 1320-1326 (2001).

348. M. Guadalupe, E. Reay, S. Sankaran, T. Prindiville, J. Flamm, A. McNeil, and S. Dandekar, Severe CD4+ T-cell depletion in gut lymphoid tissue during primary human immunodeficiency virus type 1 infection and substantial delay in restoration following highly active antiretroviral therapy, J. Virol. 77, 11708-11717 (2003).

349. J. M. Brenchley, T. W. Schacker, L. E. Ruff, D. A. Price, J. H. Taylor, G. J. Beilman, P. L. Nguyen, A. Khoruts, M. Larson, A. T. Haase, and D. C. Douek, CD4+ T-cell depletion during all stages of HIV disease occurs predominantly in the gastrointestinal tract, J. Exp. Med. 200, 749-759 (2004).

350. S. Mehandru, M. A. Poles, K. Tenner-Racz, A. Horowitz, A. Hurley, C. Hogan, D. Boden, P. Racz, and M. Markowitz, Primary HIV-1 infection is associated with preferential depletion of CD4+ T lymphocytes from effector sites in the gastrointestinal tract, J. Exp. Med. 200, 761-770 (2004).

351. G. Pantaleo, O. J. Cohen, T. Schacker, M. Vaccarezza, C. Graziosi, G. P. Rizzardi, J. Kahn, C. H. Fox, S. M. Schnittman, D. H. Schwartz, L. Corey, and A. S. Fauci, Evolutionary pattern of human immunodeficiency virus (HIV) replication and distribution in lymph nodes following primary infection: implications for antiviral therapy, Nat. Med. 4, 341-345 (1998).

352. G. A. Dean, G. H. Reubel, P. F. Moore, and N. C. Pedersen, Proviral burden and infection kinetics of feline immunodeficiency virus in lymphocyte subsets of blood and lymph node, J. Virol. 70, 5165-5169 (1996).

353. B. Hurtrel, L. Chakrabarti, M. Hurtrel, J. M. Bach, J. P. Ganiere, and L. Montagnier, Early events in lymph nodes during infection with SIV and FIV, Res. Virol. 145, 221-227 (1994).

354. G. A. Dean, G. H. Reubel, and N. C. Pedersen, Simian immunodeficiency virus infection of CD8+ lymphocytes in vivo, J. Virol. 70, 5646-5650 (1997).

355. A. De Maria, G. Pantaleo, S. M. Schnittman, J. J. Greenhouse, M. Baseler, J. M. Orenstein, and A. Fauci, Infection of CD8+ T lymphocytes with HIV. Requirement for interaction with infected CD4+ cells and induction of infectious virus from chronically infected CD8+ cells, J. Immunol. 146, 2220-2226 (1991).

356. A. Joshi, T. W. Vahlenkamp, H. Garg, W. A. Tompkins, and M. B. Tompkins, Preferential replication of FIV in activated CD4(+)CD25(+) T-cells independent of cellular proliferation, Virology 321, 307-322 (2004).

357. T. W. Vahlenkamp, M. B. Tompkins, and W. A. Tompkins, Feline immunodeficiency virus infection phenotypically and functionally activates immunosuppressive CD4+CD25+ T regulatory cells, J. Immunol. 172, 4752-4761 (2004).

358. K. Hartmann, Feline immunoderficiency virus infection-an overview, Vet. J. 155, 123-137 (1998).

359. A. H. Sparkes, C. D. Hopper, W. G. Millard, T. J. Gruffydd-Jones, and D. A. Harbour, Feline immunodeficiency virus infection. Clinicopathologic findings in 90 naturally occurring cases, J. Vet. Intern. Med. 7, 85-90 (1993).

360. T. Ishida, A. Taniguchi, S. Matsumura, T. Washizu, and I. Tomoda, Long-term clinical observations on feline immunodeficiency virus infected asymptomatic carriers, Vet. Immunol. Immunopathol. 35, 15-22 (1992).

361. R. Hofmann-Lehmann, E. Holznagel, P. Ossent, and H. Lutz, Parameters of disease progression in long-term experimental feline retrovirus (feline immunodeficiency virus and feline leukemia virus) infections: hematology, clinical chemistry, and lymphocyte subsets, Clin. Diagn. Lab. Immunol. 4, 33-42 (1997).

362. E. J. Fleming, D. L. McCaw, J. A. Smith, G. M. Buening, and C. Johnson, Clinical, hematologic, and survival data from cats infected with feline immunodeficiency virus: 42 cases (1983-1988), J. Am. Vet. Med. Assoc. 199, 913-916 (1991). 
363. G. H. Shelton, M. L. Linenberger, M. T. Persik, and J. L. Abkowitz, Prospective hematologic and clinicopathologic study of asymptomatic cats with naturally acquired feline immunodeficiency virus infection, J.Vet. Intern. Med. 9, 133-140 (1995).

364. J. A. Levy, HIV and the pathogenesis of AIDS (ASM Press, Washington, DC, 1998).

365. M. D. Daniel, N. L. Letvin, P. K. Sehgal, G. Hunsmann, D. K. Schmidt, N. W. King, and R. C. Desrosiers, Long-term persistent infection of macaque monkeys with the simian immunodeficiency virus, J. Gen. Virol. 68, 3183-3189 (1987).

366. M. B. Gardner and P. A. Luciw, Animal models of AIDS, Faseb J. 3, 2593-2606 (1989).

367. Y. Inoshima, M. Kohmoto, Y. Ikeda, H. Yamada, Y. Kawaguchi, K. Tomonaga, T. Miyazawa, C. Kai, T. Umemura, and T. Mikami, Roles of the auxiliary genes and AP-1 binding site in the long terminal repeat of feline immunodeficiency virus in the early stage of infection in cats, J. Virol. 70, 8518-8526 (1996).

368. M. De Monte, H. Nonnenmacher, N. Brignon, M. Ullmann, and J. P. Martin, A multivariate statistical analysis to follow the course of disease after infection of cats with different strains of the feline immunodeficiency virus (FIV), J. Virol. Methods 103, 157-170 (2002).

369. A. P. Kourtis, C. Ibegbu, A. J. Nahmias, F. K. Lee, W. S. Clark, M. K. Sawyer, and S. Nesheim, Early progression of disease in HIV-infected infants with thymus dysfunction, N. Engl. J. Med. 335, 1431-1436 (1997).

370. A. J. Nahmias, W. S. Clark, A. P. Kourtis, F. K. Lee, G. Cotsonis, C. Ibegbu, D. Thea, P. Palumbo, P. Vink, R. J. Simonds, and S. R. Nesheim, Thymic dysfunction and time of infection predict mortality in human immunodeficiency virus-infected infants. CDC Perinatal AIDS Collaborative Transmission Study Group, J. Infect. Dis. 178, 680-685 (1998).

371. J. Meers, W. F. Robinson, G. M. del Fierro, M. A. Scoones, and M. A. Lawson, Feline immunodeficiency virus: quantification in peripheral blood mononuclear cells and isolation from plasma of infected cats, Arch. Virol. 127, 233-243 (1992).

372. K. Tokunaga, K. Shoda, Y. Nishino, S. Mori, Q. Zhong, Y. Zheng, M. Kishi, C. Ishihara, M. Kanda, and K. Ikuta, Maintenance of high virus load even after seroconversion in newborn kittens acutely infected with feline immunodeficiency virus, Vaccine 13, 1393-1398 (1995).

373. J. A. George, N. C. Pedersen, and J. Higgins, The effect of age on the course of experimental feline immunodeficiency virus infection, AIDS Res. Hum. Retroviruses 9, 897-905 (1993).

374. M. S. Orandle, P. C. Crawford, J. K. Levy, R. Udoji, G. P. Papadi, T. Ciccarone, A. Mergia, and C. M. Johnson, CD8+ thymic lymphocytes express reduced levels of $\operatorname{CD} 8 \beta$ and increased interferon $\gamma$ in cats perinatally infected with JSY3 molecular clone of feline immunodeiciecny virus, AIDS Res. Hum. Retroviruses 16, 1559-1571 (2000).

375. G. Hoffmann-Fezer, J. Thum, C. Ackley, M. Herbold, J. Mysliwietz, S. Thefeld, K. Hartmann, and W. Kraft, Decline in CD4+ cell numbers in cats with naturally acquired feline immunodeficiency virus infection, J. Virol. 66, 1484-1488 (1992).

376. D. Gebhard, J. Dow, T. Childers, J. Alvelo, M. Tompkins, and W. Tompkins, Progressive expansion of an L-selectin-negative CD8 cell with anti-feline immunodeficiency virus (FIV) suppressor function in the circulation of FIV-infected cats, J. Infect. Dis. 180, 1503-1513 (1999).

377. B. J. Willett, M. J. Hosie, J. J. Callanan, J. C. Neil, and O. Jarrett, Infection with feline immunodeficiency virus is followed by the rapid expansion of a CD8+ lymphocyte subset, Immunology 78, 1-6 (1993).

378. M. Shimojima, T. Miyazawa, M. Kohmoto, Y. Ikeda, Y. Nishimura, K. Maeda, Y. Tohya, and T. Mikami, Expansion of CD8alpha+beta- cells in cats infected with feline immunodeficiency virus, J. Gen. Virol. 79, 91-94 (1998). 
379. P. C. Crawford, G. P. Papadi, J. K. Levy, N. A. Benson, A. Mergia, and C. M. Johnson, Tissue dynamics of CD8 lymphocytes that suppress viral replication in cats infected neonatally with feline immunodeficiency virus, J. Infect. Dis. 184, 671-681 (2001).

380. A. Oxenius, H. F. Gunthard, B. Hirschel, S. Fidler, J. N. Weber, P. J. Easterbrook, J. I. Bell, R. E. Phillips, and D. A. Price, Direct ex vivo analysis reveals distinct phenotypic patterns of HIV-specific CD8+ T lymphocyte activation in response to therapeutic manipulation of virus load, Eur. J. Immunol. 31, 1115-1121 (2001).

381. J. E. Schmitz, M. A. Forman, M. A. Lifton, O. Concepcion, K. A. Reimann, C. S. Crumpacker, J. F. Daley, R. S. Gelman, and N. L. Letvin, Expression of the CD8alpha beta-heterodimer on CD8(+) T lymphocytes in peripheral blood lymphocytes of human immunodeficiency virus- and human immunodeficiency virus+ individuals, Blood 92, 198-206 (1998).

382. C. E. Mackewicz, L. C. Yang, J. D. Lifson, and J. A. Levy, Non-cytolytic CD8 T-cell antiHIV responses in primary HIV-1 infection, Lancet. 344, 1671-1673 (1994).

383. J. A. Levy, C. E. Mackewicz, and E. Barker, Controlling HIV pathogenesis: the role of the noncytotoxic anti-HIV response of CD8+ T-cells, Immunol. Today 17, 217-224 (1996).

384. M. Torten, M. Franchini, J. E. Barlough, J. W. George, E. Mozes, H. Lutz, and N. C. Pedersen, Progressive immune dysfunction in cats experimentally infected with feline immunodeficiency virus, J. Virol. 65, 2225-2230 (1991).

385. D. S. Lin, D. D. Bowman, R. H. Jacobson, M. C. Barr, M. Fevereiro, J. R. Williams, F. M. O. Noronha, F. W. Scott, and R. J. Avery, Suppression of lymphocyte blastogenesis to mitogens in cats experimentally infected with feline immunodeficiency virus, Vet. Immunol. Immunopathol. 26, 183-189 (1990).

386. Y. Hara, T. Ishida, H. Ejima, M. Tagawa, S. Motoyoshi, I. Tomoda, M. Shimizu, and K. Shichinohe, Decrease in mitogen-induced lymphocyte proliferative responses in cats infected with feline immunodeficiency virus, Nippon Juigaku Zasshi 52, 573-579 (1990).

387. S. A. Bishop, N. A. Williams, T. J. Gruffydd-Jones, D. A. Harbour, and C. R. Stokes, Impaired T-cell priming and proliferation in cats infected with feline immunodeficiency virus, AIDS 6, 287-293 (1992).

388. K. Ohno, T. Watari, R. Goitsuka, H. Tsujimoto, and A. Hasegawa, Altered surface antigen expression on peripheral blood mononuclear cells in cats infected with feline immunodeficiency virus, J. Vet. Med. Sci. 54, 517-522 (1992).

389. G. A. Dean and N. C. Pedersen, Cytokine response in multiple lymphoid tissues during the primary phase of feline immunodeficiency virus infection, J. Virol. 72, 9436-9440 (1998).

390. Y. Liang, L. C. Hudson, J. K. Levy, J. W. Ritchey, W. A. Tompkins, and M. B. Tompkins, T-cells overexpressing interferon-gamma and interleukin-10 are found in both the thymus and secondary lymphoid tissues of feline immunodeficiency virus-infected cats, J. Infect. Dis. 181, 564-575 (2000).

391. C. E. Lawrence, J. J. Callanan, B. J. Willett, and O. Jarrett, Cytokine production by cats infected with feline immunodeficiency virus: a longitudinal study, Immunology 85, 568-574 (1995).

392. L. A. Kraus, W. G. Bradley, R. W. Engelman, K. M. Brown, R. A. Good, and N. K. Day, Relationship between tumor necrosis factor alpha and feline immunodeficiency virus expressions, J. Virol. 70, 566-569 (1996).

393. J. W. Ritchey, J. K. Levy, S. K. Bliss, W. A. Tompkins, and M. B. Tompkins, Constitutive expression of types 1 and 2 cytokines by alveolar macrophages from feline immunodeficiency virus-infected cats, Vet. Immunol. Immunopathol. 79, 83-100 (2001).

394. T. Tanabe and J. K. Yamamoto, Feline immunodeficiency virus lacks sensitivity to the antiviral activity of feline IFN-gamma, J. Interferon Cytokine Res. 21, 1039-1046 (2001). 
395. G. A. Dean, J. A. Bernales, and N. C. Pedersen, Effect of feline immunodeficiency virus on cytokine response to Listeria monocytogenes in vivo, Vet. Immunol. Immunopathol. 65, 125-138 (1998).

396. G. H. Reubel, J. W. George, J. E. Barlough, J. Higgins, C. K. Grant, and N. C. Pedersen, Interaction of acute feline herpesvirus-1 and chronic feline immunodeficiency virus infections in experimentally infected specific pathogen free cats, Vet. Immunol. Immunopathol. 35, 95-119 (1992).

397. M. Torten, E. E. Sparger, B. A. Rideout, N. C. Pedersen, and P. A. Luciw, in Vaccines 90: Modern Approaches to New Vaccines Including Prevention of AIDS, edited by F. Brown, R. M. Chanock, H. S. Ginsberg, and R. A. Lerner (Cold Spring Harbor Laboratory, Cold Spring Harbor, NY, 1990), 375-378.

398. A. Taniguchi, T. Ishida, T. Washizu, and I. Tomoda, Humoral immune response to T-cell dependent and independent antigens in cats infected with feline immunodeficiency virus, J. Vet. Med. Sci. 53, 333-335 (1991).

399. G. H. Shelton, M. L. Linenberger, C. K. Grant, and J. L. Abkowitz, Hematologic manifestations of feline immunodeficiency virus infection, Blood 76, 1104-1109 (1990).

400. D. S. Lin and D. D. Bowman, Macrophage functions in cats experimentally infected with feline immunodeficiency virus and toxoplasma gondii, Vet. Immunol. Immunopathol. 33, 69-78 (1992).

401. P. Kubes, B. Heit, G. van Marle, J. B. Johnston, D. Knight, A. Khan, and C. Power, In vivo impairment of neutrophil recruitment during lentivirus infection, J. Immunol. 171, 4801-4808 (2003).

402. M. A. Hanlon, J. M. Marr, K. A. Hayes, L. E. Mathes, P. C. Stromberg, S. Ringler, S. Krakowka, and L. J. Lafrado, Loss of neutrophil and natural killer cell function following feline immunodeficiency virus infection, Viral Immunol. 6, 119-124 (1993).

403. A. L. Parodi, F. Femenia, A. Moraillon, F. Crespeau, and J. J. Fontaine, Histopathological changes in lymph nodes of cats experimentally infected with the feline immunodeficiency virus (FIV), J. Comp. Pathol. 111, 165-174 (1994).

404. D. A. Gunn-Moore, G. R. Pearson, D. A. Harbour, and C. V. Whiting, Encephalitis associated with giant cells in a cat with naturally occurring feline immunodeficiency virus infection demonstrated by in situ hybridization, Vet. Pathol. 33, 699-703 (1996).

405. J. A. Beatty, J. J. Callanan, A. Terry, O. Jarrett, and J. C. Neil, Molecular and immunophenotypical characterization of a feline immunodeficiency virus (FIV)-associated lymphoma: a direct role for FIV in B-lymphocyte transformation?, J. Virol. 72, 767-771 (1998).

406. J. A. Beatty, C. E. Lawrence, J. J. Callanan, C. K. Grant, E. A. Gault, J. C. Neil, and O. Jarrett, Feline immunodeficiency virus (FIV)-associated lymphoma: a potential role for immune dysfunction in tumourigenesis, Vet. Immunol. Immunopathol. 65, 309-322 (1998).

407. J. A. Beatty, A. Terry, J. MacDonald, E. Gault, S. Cevario, S. J. O'Brien, E. Cameron, and J. C. Neil, Feline immunodeficiency virus integration in B-cell lymphoma identifies a candidate tumor suppressor gene on human chromosome 15q15, Cancer Res. 62, $7175-7180$ (2002).

408. Y. Endo, K. W. Cho, K. Nishigaki, Y. Momoi, Y. Nishimura, T. Mizuno, Y. Goto, T. Watari, H. Tsujimoto, and A. Hasegawa, Molecular characteristics of malignant lymphomas in cats naturally infected with feline immunodeficiency virus, Vet. Immunol. Immunopathol. 57, 153-167 (1997).

409. A. Poli, F. Abramo, F. Baldinotti, M. Pistello, L. Da Prato, and M. Bendinelli, Malignant lymphoma associated with experimentally induced feline immunodeficiency virus infection, J. Comp. Path. 10, 319-328 (1994).

410. A. A. Lackner, P. Vogel, R. A. Ramos, J. D. Kluge, and M. Marthas, Early events in tissues during infection with pathogenic (SIVmac239) and nonpathogenic (SIVmac1A11) molecular clones of simian immunodeficiency virus, Am. J. Pathol. 145, 428-439 (1994). 
411. H. L. Ioachim, C. W. Lerner, and M. L. Tapper, The lymphoid lesions associated with the acquired immunodeficiency syndrome, J. Am. Surg. Pathol. 7, 543-553 (1983).

412. P. N. Fultz, H. M. McClure, D. C. Anderson, and W. M. Switzer, Identification and biologic characterization of an acutely lethal variant of simian immunodeficiency virus from sooty mangabeys (SIV/SMM), AIDS Res. Hum. Retroviruses 5, 397-409 (1989).

413. M. L. Bonyhadi, L. Rabin, S. Salimi, D. A. Brown, J. Kosek, J. M. McCune, and H. Kaneshima, HIV induces thymus depletion in vivo, Nature 363, 728-732 (1993).

414. F. Miedema, Immunological abnormalities in the natural history of HIV infection: mechanisms and clinical relevance, Immunodefic. Rev. 3, 173-193 (1992).

415. E. M. Poeschla and D. J. Looney, CXCR4 is required by a nonprimate lentivirus: heterologous expression of feline immunodeficiency virus in human, rodent, and feline cells, J. Virol. 72, 6858-6866 (1998).

416. S. Lombardi, C. Massi, E. Indino, C. La Rosa, P. Mazzetti, M. L. Falcone, P. Rovero, A. Fissi, O. Pieroni, P. Bandecchi, F. Esposito, F. Tozzini, M. Bendinelli, and C. Garzelli, Inhibition of feline immunodeficiency virus infection in vitro by envelope glycoprotein synthetic peptides, Virology 220, 274-284 (1996).

417. D. C. Bragg, R. B. Meeker, B. A. Duff, R. V. English, and M. B. Tompkins, Neurotoxicity of FIV and FIV envelope protein in feline cortical cultures, Brain Res. 816, 431-417 (1999).

418. C. M. Johnson, N. A. Benson, and G. P. Papadi, Apoptosis and CD4+ lymphocyte depletion following feline immunodeficiency virus infection of a T-lymphocyte cell line, Vet. Pathol. 33, 195-203 (1996).

419. T. Mizuno, Y. Goto, K. Baba, K. Masuda, K. Ohno, and H. Tsujimoto, TNF- $\alpha$-induced cell death in feline immunodeficiency virus-infected cells is mediated by the caspase cascade, Virology 287, 446-455 (2001).

420. K. Ohno, Y. Okamoto, T. Miyazawa, T. Mikami, T. Watari, R. Goitsuka, H. Tsujimoto, and A. Hasegawa, Induction of apoptosis in a T lymphoblastoid cell line infected with feline immunodeficiency virus, Arch. Virol. 135, 153-158 (1994).

421. K. Ohno, T. Nakano, Y. Matsumoto, T. Watari, R. Goitsuka, H. Nakayama, H. Tsujimoto, and A. Hasegawa, Apoptosis induced by tumor necrosis factor in cells chronically infected with feline immunodeficiency virus, J. Virol. 67, 2429-2433 (1993).

422. E. Holznagel, R. Hofmann-Lehmann, C. M. Leutenegger, K. Allenspach, S. W. Huettner, U. Forster, E. Niederer, H. Joller, B. J. Willett, U. Hummel, G. L. Rossi, J. Schupbach, and H. Lutz, The role of in vitro-induced lymphocyte apoptosis in feline immunodeficiency virus infection: correlation with different markers of disease progression, J. Virol. 72, 9025-9033 (1998).

423. S. A. Bishop, T. J. Gruffydd-Jones, D. A. Harbour, and C. R. Stokes, Programmed cell death (apoptosis) as a mechanism of cell death in peripheral blood mononuclear cells from cats infected with feline immunodeficiency virus (FIV), Clin. Exp. Immunol. 93, 65-71 (1993).

424. Y. Momoi, T. Mizumno, Y. Nishimura, Y. Endo, K. Ohno, T. Watari, R. Goitsuka, H. Tsujimoto, and A. Hasegawa, Detection of apoptosis induced in peripheral blood lymphocytes from cats infected with feline immunodeficiency virus, Arch. Virol. 141, 1651-1659 (1996).

425. A. Guiot, D. Rigal, and G. Chappuis, Spontanious programmed cell death (PCD) process of lymphocytes of FIV-infected cats: cellular targets and modulation, Vet. Immunol. Immunopathol. 58, 93-106 (1997).

426. G. Sarli, S. L. Della, L. Zaccaro, M. Bendinelli, G. Piedimonte, and P. Marcato, Apoptotic fraction in lymphoid tissue of FIV-infected SPF cats, Vet. Immunol. Immunopathol. 64, 33-44 (1998).

427. H. Garg, A. Joshi, and W. A. Tompkins, Feline immunodeficiency virus envelope glycoprotein mediates apoptosis in activated PBMC by a mechanism dependent on gp41 function, Virology 330, 424-436 (2004). 
428. J. Yamazaki, N. Hasebe, S. Nagafuchi, K. Baba, H. Tsujimoto, R. Kano, and A. Hasegawa, Expression of apoptosis-related gene mRNAs in feline T-cells infected with feline immunodeficiency virus (FIV), Vet. Microbiol. 101, 1-8 (2004).

429. T. Mizuno, Y. Goto, K. Baba, Y. Momoi, Y. Endo, Y. Nishimura, K. Masuda, K. Ohno, and H. Tsujimoto, Quantitative analysis of Fas and Fas ligand mRNAs in a feline T-lymphoid cell line after infection with feline immunodeficiency virus and primary peripheral blood mononuclear cells obtained from cats infected with the virus., Vet. Immunol. Immunopathol. 93, 117-123 (2003).

430. G. Piedimonte, R. Crinelli, L. D. Salda, D. Corsi, M. G. Pennisi, L. Kramer, A. Casabianca, G. Sarli, M. Bendinelli, P. S. Marcato, and M. Magnani, Protein degradation and apoptotic death in lymphocytes during FIV infection: Activation of the ubiquitinproteasome proteolytic system, Exper. Cell Res. 248, 381-390 (1999).

431. E. Mortola, Y. Endo, T. Mizuno, K. Ohno, T. Watari, H. Tsujimoto, and A. Hasegawa, Effect of interleukin-12 and interleukin-10 on the virus replication and apoptosis in Tcells infected with feline immunodeficiency virus, J. Vet. Med. Sci. 60, 1181-1185 (1998).

432. M. E. Bull, T. W. Vahlenkamp, J. L. Dow, E. W. Collisson, B. J. Winslow, A. P. Phadke, M. B. Tompkins, and W. A. Tompkins, Spontaneous T-cell apoptosis in feline immunodeficiency virus (FIV)-infected cats is inhibited by IL2 and anti-B7.1 antibodies, Vet. Immunol. Immunopathol. 99, 25-37 (2004).

433. I. S. Choi, H. S. Yoo, and E. W. Collisson, Evaluation of expression patterns of feline CD28 and CTLA-4 in feline immunodeficiency virus (FIV)-infected and FIV antigeninduced PBMC, J. Vet. Sci. 1, 97-103 (2004).

434. M. B. Tompkins, M. E. Bull, J. L. Dow, J. M. Ball, E. W. Collisson, B. J. Winslow, A. P. Phadke, T. W. Vahlenkamp, and W. A. Tompkins, Feline immunodeficiency virus infection is characterized by B7+CTLA4+ T-cell apoptosis, J. Infect. Dis. 185, 1077-1093 (2002).

435. T. W. Vahlenkamp, M. E. Bull, J. L. Dow, J. M. Ball, E. W. Collisson, B. J. Winslow, A. P. Phadke, W. A. Tompkins, and M. B. Tompkins, B7+CTLA4+ T-cells engage in T-T interactions that mediate apoptosis: a model for lentivirus-induced T-cell depletion, Vet. Immunol. Immunopathol. 98, 203-214 (2004).

436. Q. Leng, Z. Bentwich, E. Magen, A. Kalinkovich, and G. Borkow, CTLA-4 upregulation during HIV infection: association with anergy and possible target for therapeutic intervention, AIDS 16, 519-529 (2002).

437. D. E. Lewis, L. Yang, W. Luo, X. Wang, and J. R. Rodgers, HIV-specific cytotoxic T lymphocyte precursors exist in a CD28-CD8+ T-cell subset and increase with loss of CD4 T-cells, AIDS 13, 1029-1033, (1999).

438. E. N. Mugnaini, A. Spurkland, T. Egeland, M. Sannes, and J. E. Brinchmann, Demonstration of identical expanded clones within both CD8+CD28+ and CD8+CD28- T-cell subsets in HIV type 1-infected individuals, Eur. J. Immunol. 28, 1738-1742 (1998).

439. O. K. Haffar, M. D. Smithgall, J. G. Wong, J. Bradshaw, and P. S. Linsley, Human immunodeficiency virus type 1 infection of CD4+ T-cells down-regulates the expression of CD28: effect on T-cell activation and cytokine production, Clin. Immunol. Immunopathol. 77, 262-270 (1995).

440. J. E. Brinchmann, J. H. Dobloug, B. H. Heger, L. L. Haaheim, M. Sannes, and T. Egeland, Expression of costimulatory molecule CD28 on T-cells in human immunodeficiency virus type 1 infection: functional and clinical correlations, J. Infect. Dis. 169, 730-738 (1994).

441. W. E. Johnson, J. D. Lifson, S. M. Lang, R. P. Johnson, and R. C. Desrosiers, Importance of B-cell responses for immunological control of variant strains of simian immunodeficiency virus, J. Virol. 77, 375-381 (2003).

442. J. E. Schmitz, M. J. Kuroda, S. Santra, V. G. Sasseville, M. A. Simon, M. A. Lifton, P. Racz, K. Tenner-Racz, M. Dalesandro, B. J. Scallon, J. Ghrayeb, M. A. Forman, M. D. C., E. P. Rieber, N. L. Letvin, and K. A. Reimann, Control of viremia in simian immunodeficiency virus infection by CD8+ lymphocytes, Science 283, 857-860 (1999). 
443. J. E. Schmitz, M. J. Kuroda, S. Santra, M. A. Simon, M. A. Lifton, W. Lin, R. Khunkhun, M. Piatak, J. Lifson, G. Grosschupff, R. S. Gelman, P. Racz, K. Tenner-Racz, K. A. Mansfield, N. L. Letvin, D. C. Montefiori, and K. A. Reimann, Effect of humoral immune responses on controlling viremia during primary infection of rhesus monkeys with simian immunodeficiency virus, J. Virol. 77, 2165-2173 (2003).

444. T. P. O'Connor, S. Tanguay, R. Steinman, R. A. Smith, M. C. Barr, J. K. Yamamoto, N. C. Pedersen, P. R. Andersen, and Q. J. Tonelli, Development and evaluation of immunoassay for detection of antibodies to the feline T-lymphotropic lentivirus (feline immunodeficiency virus). J. Clin. Microbiol. 27, 474-479 (1989).

445. M. J. Hosie and O. Jarrett, Serological responses of cats to feline immunodeficiency virus, AIDS 4, 215-220 (1990).

446. S. Lombardi, M. Bendinelli, and C. Garzelli, Detection of B epitopes on the p24 gag protein of feline immunodeficiency virus by monoclonal antibodies, AIDS Res. Hum. Retroviruses 9, 141-146 (1993).

447. A. Trkola, H. Kuster, C. Leemann, A. Oxenius, C. Fagard, H. Furrer, M. Battegay, P. Vernazza, E. Bernasconi, R. Weber, B. Hirschel, S. Bonhoeffer, H. F. Gunthard, and S. H. C. Study, Humoral immunity to HIV-1: kinetics of antibody responses in chronic infection reflects capacity of immune system to improve viral set point, Blood 104, 1784-1792 (2004).

448. P. W. Parren, J. P. Moore, D. R. Burton, and Q. J. Sattentau, The neutralizing antibody response to HIV-1: viral evasion and escape from humoral immunity AIDS, AIDS 13, S137-S162 (1999).

449. D. R. Burton, R. A. Williamson, and P. W. Parren, Antibody and virus: binding and neutralization, Virology 270, 1-3 (2000).

450. A. Avrameas, A. Strosberg, A. Moraillon, P. Sonigo, and G. Pancino, Serological diagnosis of feline immunodeficiency virus infection based on synthetic peptides from Env glycoproteins, Res Virol. 144, 209-218 (1993).

451. S. Lombardi, C. Garzelli, C. La Rosa, L. Zaccaro, S. Specter, G. Malvaldi, F. Tozzini, F. Esposito, and M. Bendinelli, Identification of a linear neutralization site within the third variable region of the feline immunodeficiency virus envelope, J. Virol. 67, 4742-4749 (1993).

452. G. Pancino, C. Chappey, W. Saurin, and P. Sonigo, B epitopes and selection pressures in feline immunodeficiency virus envelope glycoproteins, J. Virol. 67, 664-672 (1993).

453. C. Massi, S. Lombardi, E. Indino, D. Matteucci, C. La Rosa, F. Esposito, C. Garzelli, and M. Bendinelli, Most potential linear B cell epitopes of Env glycoproteins of feline immunodeficiency virus are immunogenically silent in infected cats, AIDS Res. Hum. Retroviruses 13, 1121-1129 (1997).

454. J. Richardson, I. Fossati, A. Moraillon, S. Castelot, P. Sonigo, and G. Pancino, Neutralization sensitivity and accessibility of continuous B cell epitopes of the feline immunodeficiency virus envelope, J. Gen. Virol. 77, 759-771 (1996).

455. K. H. Siebelink, G. F. Rimmelzwaan, M. L. Bosch, R. H. Meloen, and A. D. Osterhaus, A single amino acid substitution in hypervariable region 5 of the envelope protein of feline immunodeficiency virus allows escape from virus neutralization, J. Virol. 67, 2202-2208 (1993).

456. K. H. Siebelink, W. Huisman, J. A. Karlas, G. F. Rimmelzwaan, M. L. Bosch, and A. D. Osterhaus, Neutralization of feline immunodeficiency virus by polyclonal feline antibody: simultaneous involvement of hypervariable regions 4 and 5 of the surface glycoprotein, J. Virol. 69, 5124-5127 (1995).

457. G. Freer, S. Giannecchini, A. Tissot, M. F. Bachmann, P. Rovero, P. F. Serres, and M. Bendinelli, Dissection of seroreactivity against the tryptophan-rich motif of the feline immunodeficiency virus transmembrane glycoprotein, Virology 322, 360-369 (2004). 
458. M. Bendinelli, M. Pistello, D. Del Mauro, G. Cammarota, F. Maggi, A. Leonildi, S. Giannecchini, C. Bergamini, and D. Matteucci, During readaptation in vivo, a tissue culture-adapted strain of feline immunodeficiency virus reverts to broad neutralization resistance at different times in individual hosts but through changes at the same position of the surface glycoprotein, J. Virol. 75, 4584-4593 (2001).

459. G. Cammarota, D. Matteucci, M. Pistello, E. Nicoletti, S. Giannecchini, and M. Bendinelli, Reduced sensitivity to strain-specific neutralization of laboratory-adapted feline immunodeficiency virus after one passage in vivo: association with amino acid substitutions in the V4 region of the surface glycoprotein, AIDS Res. Hum. Retroviruses 12, 173-175 (1996).

460. S. Giannecchini, D. Matteucci, A. Ferrari, M. Pistello, and M. Bendinelli, Feline immunodeficiency virus-infected cat sera associated with the development of broad neutralization resistance in vivo drive similar reversions in vitro, J. Virol. 75, 8868-8873 (2001).

461. M. Pistello, D. Matteucci, S. Giannecchini, F. Bonci, O. Sichi, S. Presciuttini, and M. Bendinelli, Evolution of two amino acid positions governing broad neutralization resistance in a strain of feline immunodeficiency virus over 7 years of persistence in cats, Clin. Diagn. Lab. Immunol. 10, 1109-1116 (2003).

462. K. H. J. Siebelink, E. Tijhaar, R. C. Huisman, W. Huisman, A. De Ronde, I. H. Darby, M. J. Francis, G. F. Rimmelzwaan, and A. D. Osterhaus, Enhancement of feline immunodeficiency virus infection after immunization with envelope glycoprotein subunit vaccines, J. Virol. 69, 3704-3711 (1995).

463. D. R. Burton, R. C. Desrosiers, R. W. Doms, W. C. Koff, P. D. Kwong, J. P. Moore, G. J. Nabel, J. Sodroski, I. A. Wilson, and R. T. Wyatt, HIV vaccine design and the neutralizing antibody problem, Nat. Immunol. 5, 233-236 (2004).

464. M. B. Zwick, R. Jensen, S. Church, M. Wang, G. Stiegler, R. Kunert, H. Katinger, and D. R. Burton, Anti-human immunodeficiency virus type 1 (HIV-1) antibodies 2F5 and 4 E10 require surprisingly few crucial residues in the membrane-proximal external region of glycoprotein gp41 to neutralize HIV-1, J. Virol. 79, 1252-1261 (2005).

465. J. Richardson, A. Moraillon, F. Crespeau, S. Baud, P. Sonigo, and G. Pancino, Delayed infection after immunization with a peptide from the transmembrane glycoprotein of the feline immunodeficiency virus, J. Virol. 72, 2406-2415 (1998).

466. N. L. Haigwood and L. Stamatatos, Role of neutralizing antibodies in HIV infection, AIDS 17 Suppl. 4, S67-71 (2003).

467. R. A. Rasmussen, R. Hofmann-Lehmann, P. L. Li, J. Vlasak, J. E. Schmitz, K. A. Reimann, M. J. Kuroda, N. L. Letvin, D. C. Montefiori, H. M. McClure, and R. M. Ruprecht, Neutralizing antibodies as a potential secondary protective mechanism during chronic SHIV infection in CD8+ T-cell-depleted macaques, AIDS 16, 829-838 (2002).

468. R. Pu, S. Okada, E. R. Little, B. Xu, W. V. Stoffs, and J. K. Yamamoto, Protection of neonatal kittens against feline immunodeficiency virus infection with passive maternal antiviral antibodies, AIDS 9, 235-242 (1995).

469. K. K. Van Rompay, C. J. Berardi, S. Dillard-Telm, R. P. Tarara, D. R. Canfield, C. R. Valverde, D. C. Montefiori, K. S. Cole, R. C. Montelaro, C. J. Miller, and M. L. Marthas, Passive immunization of newborn rhesus macaques prevents oral simian immunodeficiency virus infection, J. Infect. Dis. 177, 1247-1259 (1998).

470. F. Ferrantelli, R. A. Rasmussen, K. A. Buckley, P. L. Li, T. Wang, D. C. Montefiori, H. Katinger, G. Stiegler, D. C. Anderson, H. M. McClure, and R. M. Ruprecht, Complete protection of neonatal rhesus macaques against oral exposure to pathogenic simianhuman immunodeficiency virus by human anti-HIV monoclonal antibodies, J. Infect. Dis. 189, 2149-2153 (2004).

471. Q. J. Sattentau, Neutralization of HIV-1 by antibody, Curr. Opin. Immunol. 8, 540-545 (1996). 
472. W. Huisman, J. A. Karlas, K. H. Siebelink, R. C. Huisman, A. de Ronde, M. J. Francis, G. F. Rimmelzwaan, and A. D. Osterhaus, Feline immunodeficiency virus subunit vaccines that induce virus neutralising antibodies but no protection against challenge infection, Vaccine 16, 181-187 (1998).

473. F. Baldinotti, D. Matteucci, P. Mazzetti, C. Giannelli, P. Bandecchi, F. Tozzini, and M. Bendinelli, Serum neutralization of feline immunodeficiency virus is markedly dependent on passage history of the virus and host system, J. Virol. 68, 4572-4579 (1994).

474. D. Del Mauro, D. Matteucci, S. Giannecchini, F. Maggi, M. Pistello, and M. Bendinelli, Autologous and heterologous neutralization analyses of primary feline immunodeficiency virus isolates, J. Virol. 72, 2199-2207 (1998).

475. M. J. Burkhard, C. K. Mathiason, T. Bowdre, and E. A. Hoover, Feline immunodeficiency virus Gag- and Env-specific immune responses after vaginal versus intravenous infection, AIDS Res. Hum. Retroviruses 17, 1767-1778 (2001).

476. T. Hohdatsu, S. Fujimori, M. Maeki, N. Suma, K. Motokawa, S. Okada, and H. Koyama, Virus neutralizing antibody titer to feline immunodeficiency virus isolates of subtypes A, B and D in experimentally or naturally infected cats, J. Vet. Med. Sci. 59, 377-381 (1997).

477. Y. Inoshima, T. Miyazawa, M. Kohmoto, Y. Ikeda, E. Sato, T. Hohdatsu, C. K. MathiasonDubard, E. A. Hoover, and T. Mikami, Cross virus neutralizing antibodies against feline immunodeficiency virus genotypes A, B, C, D and E, Arch. Virol. 143, 157-162 (1998).

478. J. A. Levy, The search for the CD8+ cell anti-HIV factor (CAF), Trends Immunol. 24, 628-632 (2003).

479. J. D. Lifson, J. L. Rossio, M. J. Piatak, T. Parks, L. Li, R. Kiser, V. Coalter, B. Fisher, B. M. Flynn, S. Czajak, V. M. Hirsch, K. A. Reimann, J. E. Schmitz, J. Ghrayeb, N. Bischofberger, M. A. Nowak, R. C. Desrosiers, and D. Wodarz, Role of CD8(+) lymphocytes in control of simian immunodeficiency virus infection and resistance to rechallenge after transient early antiretroviral treatment, J. Virol. 75, 10187-10199 (2001).

480. J. N. Flynn, C. A. Cannon, J. C. Neil, and O. Jarrett, Vaccination with a feline immunodeficiency virus multiepitopic peptide induces cell-mediated and humoral immune responses in cats, but does not confer protection, J. Virol. 71, 7586-7592 (1997).

481. G. A. Dean, A. LaVoy, and M. J. Burkhard, Peptide mapping of feline immunodeficiency virus by IFN-gamma ELISPOT, Vet. Immunol. Immunopathol. 100, 49-59 (2004).

482. J. A. Beatty, B. J. Willett, E. A. Gault, and O. Jarrett, A longitudinal study of feline immunodeficiency virus-specific cytotoxic T lymphocytes in experimentally infected cats, using antigen-specific induction, J. Virol. 70, 6199-6206 (1996).

483. S. Finerty, C. R. Stokes, T. J. Gruffydd-Jones, T. J. Hillman, N. A. Reeves, C. V. Whiting, W. M. Schaaper, K. Dalsgaard, and D. A. Harbour, Mucosal immunization with experimental feline immunodeficiency virus (FIV) vaccines induces both antibody and T-cell responses but does not protect against rectal FIV challenge, Vaccine 18, 3254-3265 (2000).

484. J. N. Flynn, J. A. Beatty, C. A. Cannon, E. B. Stephens, M. J. Hosie, J. C. Neil, and O. Jarrett, Involvement of gag- and env-specific cytotoxic $\mathrm{T}$ lymphocytes in protective immunity to feline immunodeficiency virus, AIDS Res. Hum. Retroviruses 11, 1107-1113 (1995).

485. S. Giannecchini, P. Isola, O. Sichi, D. Matteucci, M. Pistello, L. Zaccaro, D. Del Mauro, and M. Bendinelli, AIDS vaccination studies using an ex vivo feline immunodeficiency virus model: failure to protect and possible enhancement of challenge infection by four cell-based vaccines prepared with autologous lymphoblasts, J. Virol. 76, 6882-6892 (2002).

486. C. R. Jeng, R. V. English, T. Childers, M. B. Tompkins, and W. A. Tompkins, Evidence for CD8+ antiviral activity in cats infected with feline immunodeficiency virus, J. Virol. 70, 2474-2480 (1996). 
487. R. Pu, J. Coleman, M. Omori, M. Arai, T. Hohdatsu, C. Huang, T. Tanabe, and J. K. Yamamoto, Dual-subtype FIV vaccine protects cats against in vivo swarms of both homologous and heterologous subtype FIV isolates, AIDS 15, 1225-1237 (2001).

488. R. Pu, M. Omori, S. Okada, S. L. Rine, B. A. Lewis, E. Lipton, and J. K. Yamamoto, MHCrestricted protection of cats against FIV infection by adoptive transfer of immune cells from FIV-vaccinated donors, Cell. Immunol. 198, 30-43 (1999).

489. J. Sirriyah, G. A. Dean, A. LaVoy, and M. J. Burkhard, Assessment of CD4+ and CD8+ IFN-gamma producing cells by ELISPOT in naive and FIV-infected cats, Vet. Immunol. Immunopathol. 102, 77-84 (2004).

490. W. Song, E. W. Collisson, P. M. Billingsley, and W. C. Brown, Induction of feline immunodeficiency virus-specific cytolytic T-cell responses from experimentally infected cats, J. Virol. 66, 5409-5417 (1992).

491. W. Song, E. W. Collisson, J. Li, A. M. Wolf, J. H. Elder, C. K. Grant, and W. C. Brown, Feline immunodeficiency virus (FIV)-specific cytotoxic T lymphocytes from chronically infected cats are induced in vitro by retroviral vector-transduced feline T-cells expressing the FIV capsid protein, Virology 209, 390-399 (1995).

492. J. N. Flynn, S. T. Dunham, A. Mueller, C. Cannon, and O. Jarrett, Involvement of cytolytic and non-cytolytic T-cells in the control of feline immunodeficiency virus infection, Vet. Immunol. Immunopathol. 85, 159-170 (2002).

493. J. N. Flynn, C. A. Cannon, G. Reid, M. A. Rigby, J. C. Neil, and O. Jarrett, Induction of feline immunodeficiency virus-specific cell-mediated and humoral immune responses following immunization with a multiple antigenic peptide from the envelope V3 domain, Immunology 85, 171-175 (1995).

494. D. T. Evans, D. H. O’Connor, P. Jing, J. L. Dzuris, J. Sidney, J. da Silva, T. M. Allen, H. Horton, J. E. Venham, R. A. Rudersdorf, T. Vogel, C. D. Pauza, R. E. Bontrop, R. DeMars, A. Sette, A. L. Hughes, and D. I. Watkins, Virus-specific cytotoxic T-lymphocyte responses select for amino-acid variation in simian immunodeficiency virus Env and Nef, Nat. Med. 5, 1270-1276 (1999).

495. P. J. Goulder, R. E. Phillips, R. A. Colbert, S. McAdam, G. Ogg, M. A. Nowak, P. Giangrande, G. Luzzi, B. Morgan, A. Edwards, A. J. McMichael, and S. Rowland-Jones, Late escape from an immunodominant cytotoxic T-lymphocyte response associated with progression to AIDS, Nat. Med. 3, 212-217 (1997).

496. P. J. Goulder and B. D. Walker, The great escape-AIDS viruses and immune control, Nat. Med. 5, 1233-1235 (1999).

497. M. Omori, R. Pu, T. Tanabe, W. Hou, J. K. Coleman, M. Arai, and J. K. Yamamoto, Cellular immune responses to feline immunodeficiency virus (FIV) induced by dual-subtype FIV vaccine, Vaccine 23, 386-398 (2004).

498. T. Hohdatsu, M. Okubo, and H. Koyama, Feline CD8+ T-cell non-cytolytic anti-feline immunodefieicncy virus activity mediated by a soluble factor(s), J. Gen. Virol. 79, 2729-2735 (1998).

499. M. Shimojima, Y. Nishimura, T. Miyazawa, Y. Tohya, and H. Akashi, Phenotypic changes in CD8+ peripheral blood lymphocytes in cats infected with feline immunodeficiency virus, Microbes Infect. 5, 1171-1176 (2003).

500. S. T. Hohdatsu T., A. Yamazaki, K. Motokawa, H. Kusuhara, T. Kaneshima, and H. Koyama, CD8+ T-cells from feline immunodeficiency virus (FIV) infected cats suppress exogenous FIV replication of their peripheral blood mononuclear cells in vitro, Arch. Virol. 147, 1517-1529 (2002).

501. J. N. Flynn, C. A. Cannon, D. Sloan, J. C. Neil, and O. Jarrett, Suppression of feline immunodeficiency virus replication in vitro by a soluble factor secreted by CD8+ T lymphocytes, Immunology 96, 220-229 (1999).

502. I. S. Choi, R. Hokanson, and E. W. Collisson, Anti-feline immunodeficiency virus (FIV) soluble factor(s) produced from antigen-stimulated feline CD8(+) T lymphocytes suppresses FIV replication, J. Virol. 74, 676-683 (2000). 
503. T. Hohdatsu, A. Yamazaki, M. Yamada, H. Kusuhara, T. Kaneshima, and H. Koyama, Ability of CD8(+) T-cell anti-feline immunodeficiency virus activity correlated with peripheral CD4(+) T-cell counts and plasma viremia, Microbiol. Immunol. 47, 765-773 (2003).

504. A. P. Phadke, I. S. Choi, Z. Li, E. A. Weaver, and E. W. Collisson, The role of inducer cells in mediating in vitro suppression of feline immunodeficiency virus replication, Virology 320, 63-74 (2004).

505. M. S. Wyand, K. H. Manson, M. Garcia-Moll, D. Montefiori, and R. C. Desrosiers, Vaccine protection by a triple deletion mutant of simian immunodeficiency virus, J. Virol. 70, 3724-3733 (1996).

506. M. D. Daniel, F. Kirchhoff, S. C. Czajak, P. K. Sehgal, and R. C. Desrosiers, Protective effects of a live attenuated SIV vaccine with a deletion in the nef gene, Science 258, 1938-1941 (1992).

507. K. Abel, L. Compton, T. Rourke, D. Montefiori, D. Lu, K. Rothaeusler, L. Fritts, K. Bost, and C. J. Miller, Simian-human immunodeficiency virus SHIV89.6-induced protection against intravaginal challenge with pathogenic SIVmac239 is independent of the route of immunization and is associated with a combination of cytotoxic T-lymphocyte and alpha interferon responses, J. Virol. 77, 3099-3118 (2003).

508. J. B. Whitney and R. M. Ruprecht, Live attenuated HIV vaccines: pitfalls and prospects, Curr. Opin. Infect. Dis. 17, 17-26 (2004).

509. T. W. Baba, Y. S. Jeong, D. Penninck, R. Bronson, M. F. Greene, and R. M. Ruprecht, Pathogenicity of live, attenuated SIV after mucosal infection of neonatal macaques, Science 267, 1820-1825 (1995).

510. T. W. Baba, V. Liska, A. H. Khimani, N. B. Ray, P. J. Dailey, D. Penninck, R. Bronson, M. F. Greene, H. M. McClure, L. N. Martin, and R. M. Ruprecht, Live attenuated, multiply deleted simain immunodeficiency virus causes AIDS in infant and adult macaques, Nature Med. 5, 194-203 (1999).

511. R. R. Amara, F. Villinger, J. D. Altman, S. L. Lydy, et al., Control of a mucosal challenge and prevention of AIDS by a multiprotein DNA/MVA vaccine, Science 292, 69-74 (2001).

512. D. H. Barouch, S. Santra, J. E. Schmitz, M. J. Kuroda, et al., Control of viremia and prevention of clinical AIDS in rhesus macaques by cytokine-augmented DNA vaccination, Science 290, 486-491 (2000).

513. R. R. Amara and H. L. Robinson, A new generation of HIV vaccines, Trends Mol. Med. 8 , 489-495 (2002).

514. E. W. Uhl, T. G. Heaton-Jones, R. Pu, and J. K. Yamamoto, FIV vaccine development and its importance to veterinary and human medicine: a review, Vet. Immunol. Immunopathol. $6714,1-20$ (2002).

515. J. Cohen, HIV/AIDS in China. Vaccine development with a distinctly Chinese flavor, Science 304, 1437 (2004).

516. Y. Kono, K. Kobayashi, and Y. Fukunaga, Immunization of horses against equine infectious anemia (EIA) with an attenuated EIA virus, Natl. Inst. Anim. Health Q. (Tokyo) 10, 113-122 (1970).

517. J. K. Levy, P. C. Crawford, and M. R. Slater, Effect of vaccination against feline immunodeficiency virus on results of serologic testing in cats, J. Am. Vet. Med. Assoc. 225, 1558-1561 (2004).

518. J. A. Karlas, K. H. Siebelink, M. A. Peer, W. Huisman, G. F. Rimmelzwaan, and A. D. Osterhaus, Accelerated viraemia in cats vaccinated with fixed autologous FIV-infected cells, Vet. Immunol. Immunopathol. 65, 353-365 (1998).

519. J. A. Karlas, K. H. Siebelink, M. A. Peer, W. Huisman, A. M. Cuisinier, G. F. Rimmelzwaan, and A. D. Osterhaus, Vaccination with experimental feline immunodeficiency virus vaccines, based on autologous infected cells, elicits enhancement of homologous challenge infection, J. Gen. Virol. 80, 761-765 (1999). 
520. M. J. Hosie, R. Osborne, G. Reid, J. C. Neil, and O. Jarrett, Enhancement after feline immunodeficiency virus vaccination, Vet. Immunol. Immunopathol. 35, 191-197 (1992).

521. W. Hesselink, P. J. Sondermeijer, H. Pouwels, E. Verblakt, and C. Dhore, Vaccination of cats against feline immunodeficiency virus (FIV): a matter of challenge, Vet. Microbiol. 69, 109-110 (1999).

522. M. J. Hosie, R. Osborne, J. K. Yamamoto, J. C. Neil, and O. Jarrett, Protection against homolgous but not heterologous challenge induced by inactivated feline immunodeficiency virus vaccines, J. Virol. 69, 1253-1255 (1995).

523. M. J. Hosie, T. Dunsford, D. Klein, B. J. Willett, C. Cannon, R. Osborne, J. MacDonald, N. Spibey, N. A. Mackay, O. Jarrett, and J. C. Neil, Vaccination with inactivated virus but not viral DNA reduces virus load following challenge with a heterologous and virulent isolate of feline immunodeficiency virus, J. Virol. 74, 9403-9411 (2000).

524. S. Finerty, C. R. Stokes, T. J. Gruffydd-Jones, T. J. Hillman, F. J. Barr, and D. A. Harbour, Targeted lymph node immunization can protect cats from a mucosal challenge with feline immunodeficiency virus, Vaccine 20, 49-58 (2001).

525. D. Matteucci, M. Pistello, P. Mazzetti, S. Giannecchini, D. Del Mauro, L. Zaccaro, P. Bancecchi, F. Tozzini, and M. Bendinelli, Vaccination protects against in vivo-grown feline immunodeficiency virus even in the absence of detectable neutralizing antibodies, J. Virol. 70, 617-622 (1996).

526. D. Matteucci, A. Poli, P. Mazzetti, S. Sozzi, F. Bonci, P. Isola, L. Zaccaro, S. Giannecchini, M. Calandrella, M. Pistello, S. Specter, and M. Bendinelli, Immunogenicity of an anticlade B feline immunodeficiency fixed-cell vrius vaccine in field cats, J. Virol. 74, 10911-10919 (2000).

527. T. Hohdatsu, S. Okada, K. Motokawa, C. Aizawa, J. K. Yamamoto, and H. Koyama, Effect of dual-subtype vaccine against feline immunodeficiency virus infection, Vet. Microbiol. 58, 155-165 (1997).

528. M. C. Tellier, R. Pu, D. Pollock, A. Vitsky, J. Tartaglia, E. Paoletti, and J. K. Yamamoto, Efficacy evaluation of prime-boost protocol: canarypoxvirus-based feline immunodeficiency virus (FIV) vaccine and inactivated FIV-infected cell vaccine against heterologous FIV challenge in cats, AIDS 12, 11-18 (1998).

529. S. A. Bishop, C. R. Stokes, T. J. Gruffydd-Jones, C. V. Whiting, J. E. Humphries, R. Osborne, M. Papnastasopoulou, and D. A. Harbour, Vaccination with fixed feline immunodeficiency virus (FIV) infected cells: protection, breakthrough and specificity of response, Vaccine 14, 1243-1250 (1996).

530. D. Matteucci, M. Pistello, P. Mazzetti, S. Giannecchini, D. Del Mauro, I. Lonetti, L. Zaccaro, C. Pollera, S. Specter, and M. Bendinelli, Studies of AIDS vaccination using an ex vivo feline immunodeficiency virus model: protection conferred by a fixed-cell vaccination against cell-free and cell-associated challenge differs in duration and is not easily boosted, J. Virol. 71, 8368-8376 (1997).

531. M. J. Hosie, T. H. Dunsford, A. de Ronde, B. J. Willett, C. A. Cannon, J. C. Neil, and O. Jarrett, Suppression of virus burden by immunization with feline immunodeficiency virus Env protein, Vaccine 14, 405-411 (1996).

532. P. Mazzetti, S. Giannecchini, D. Del Mauro, D. Matteucci, P. Portincasa, A. Merico, C. Chezzi, and M. Bendinelli, AIDS vaccination studies using an ex vivo feline immunodeficiency virus model: detailed analysis of the humoral immune response to a protective vaccine, J. Virol. 73, 1-10 (1999).

533. D. Matteucci, M. Pistello, P. Mazzetti, S. Giannecchini, P. Isola, A. Merico, L. Zaccaro, and A. Rizzuti, AIDS vaccination studies using feline immunodeficiency virus suppresses viraemia levels following intravaginal challenge with infected cells but not following intravenous challenge with cell-free virus, Vaccine 18, 119-130 (1999).

534. S. Giannecchini, D. Del Mauro, D. Matteucci, and M. Bendinelli, AIDS vaccination studies using an ex vivo feline immunodeficiency virus model: reevaluation of neutral- 
izing antibody levels elicited by a protective and a nonprotective vaccine after removal of antisubstrate cell antibodies, J. Virol. 75, 4424-4429 (2001).

535. J. N. Flynn, P. Keating, M. J. Hosie, M. Mackett, E. B. Stephens, J. A. Beatty, J. C. Neil, and O. Jarrett, Env-specific CTL predominate in cats protected from feline immunodeficiency virus infection by vaccination, J. Immunol. 157, 3658-3665 (1996).

536. M. C. Tellier, J. Soos, R. Pu, D. Pollock, and J. K. Yamamoto, Development of FIVspecific cytolytic T-lymphocyte responses in cats upon immunisation with FIV vaccines, Vet. Microbiol. 57, 1-11 (1997).

537. M. J. Hosie and J. N. Flynn, Feline immunodefiiency virus vaccination: characterization of the immune correlates of protection, J. Virol. 70, 7561-7568 (1996).

538. W. L. Chan, A. Rodgers, R. D. Hancock, F. Taffs, P. Kitchin, G. Farrar, and F. Y. Liew, Protection in simian immunodeficiency virus-vaccinated monkeys correlates with anti-HLA class I antibody response, J. Exp. Med. 176, 1203-1207 (1992).

539. E. J. Stott, Anti-cell antibody in macaques, Nature 353, 393 (1991).

540. N. A. Doria-Rose, C. Ohlen, P. Polacino, C. C. Pierce, M. T. Hensel, L. Kuller, T. Mulvania, D. C. Anderson, P. D. Greenberg, S. L. Hu, and N. Haigwood, Multigene DNA priming-boosting vaccines protect macaques from acute CD4+-T-cell depletion after simian-human immunodeficiency virus SHIV89.6P mucosal challenge, J. Virol. 77, 11563-11577 (2003).

541. R. L. Willey, R. Byrum, M. Piatak, Y. B. Kim, M. W. Cho, J. L. Rossio, J. Bess, T. Igarashi, Y. Endo, L. O. Arthur, J. D. Lifson, and M. A. Martin, Control of viremia and prevention of simian-human immunodeficiency virus-induced disease in rhesus macaques immunized with recombinant vaccinia viruses plus inactivated simian immunodeficiency virus and human immunodeficiency virus type 1 particles, J. Virol. 77, 1163-1174 (2003).

542. E. J. Verschoor, M. J. Willemse, J. G. Stam, A. L. van Vliet, H. Pouwels, S. K. Chalmers, M. C. Horzinek, P. J. Sondermeijer, W. Hesselink, and A. de Ronde, Evaluation of subunit vaccines against feline immunodeficiency virus infection, Vaccine 14, 285-289 (1996).

543. A. Cuisinier, A. Meyer, B. Chatrenet, A. S. Verdier, and A. Aubert, Attempt to modify the immune response developed against FIV gp120 protein by preliminary FIV DNA injection, Vaccine 17, 415-425 (1999).

544. H. Lutz, R. Hofmann-Lehmann, K. Bauer-Pham, E. Holznagel, F. Tozzini, M. Bendinelli, G. Reubel, A. Aubert, D. Davis, D. Cox, and E. Young, FIV vaccine studies. I. Immune response to recombinant FIV env gene products and outcome after challenge infection, Vet. Immunol. Immunopathol. 46, 103-113 (1995).

545. C. M. Leutenegger, R. Hofmann-Lehmann, E. Holznagel, A. M. Cuisinier, C. Wolfensberger, V. Duquesne, J. Cronier, K. Allenspach, A. Aubert, P. Ossent, and H. Lutz, Partial protection by vaccination with recombinant feline immunodeficiency virus surface glycoproteins, AIDS Res. Hum. Retroviruses 14, 275-283 (1998).

546. N. L. Letvin, Progress in the development of an HIV-1 vaccine, Science 280, 1875-1880 (1998).

547. L. Chiarantini, D. Matteucci, M. Pistello, U. Mancini, P. Mazzetti, C. Massi, S. Giannecchini, I. Lonetti, M. Magnani, and M. Bendinelli, AIDS vaccination studies using an ex vivo feline immunodeficiency virus model: homologous erythrocytes as a delivery system for preferential immunization with putative protective antigens, Clin. Diagn. Lab. Immunol. 5, 235-241 (1998).

548. S. Zolla-Pazner, Identifying epitopes of HIV-1 that induce protective antibodies, Nat. Rev. Immunol. 4, 199-210 (2004).

549. M. J. Hosie, J. N. Flynn, M. A. Rigby, C. Cannon, T. Dunsford, N. A. Mackay, D. Argyle, B. J. Willett, T. Miyazawa, D. E. Onions, O. Jarrett, and J. C. Neil, DNA vaccination affords significant protection against feline immunodeficiency virus infection without inducing detectable antiviral antibodies, J. Virol. 72, 7310-7319 (1998). 
550. F. S. Boretti, C. M. Leutenegger, C. N. Mislin, R. Hofmann-Lehmann, S. Koenig, M. Schroff, C. Junghans, D. Fehr, S. W. Huettner, A. Habel, J. N. Flynn, A. Aubert, N. C. Pedersen, B. Wittig, and H. Lutz, Protection against FIV challenge infection by genetic vaccination using minimalistic DNA constructs for FIV env gene and feline IL-12 expression, AIDS 14, 1749-1757 (2000).

551. C. M. Leutenegger, F. S. Boretti, C. N. Mislin, J. N. Flynn, M. Schroff, A. Habel, C. Junghans, S. A. Koenig-Merediz, B. Sigrist, A. Aubert, N. C. Pedersen, B. Wittig, and H. Lutz, Immunization of cats against feline immunodeficiency virus (FIV) infection by using minimalistic immunogenic defined gene expression vector vaccines expressing FIV gp140 alone or with feline interleukin-12 (IL-12), IL-16, or a CpG motif, J. Virol. 74, 10447-10457 (2000).

552. L. Deml, A. Bojak, S. Steck, M. Graf, J. Wild, R. Schirmbeck, H. Wolf, and R. Wagner, Multiple effects of codon usage optimization on expression and immunogenicity of DNA candidate vaccines encoding the human immunodeficiency virus type 1 Gag protein, J. Virol. 75, 10991-11001 (2001).

553. J. N. Flynn, M. J. Hosie, M. A. Rigby, N. Mackay, C. A. Cannon, T. Dunsford, J. C. Neil, and $\mathrm{O}$. Jarrett, Factors influencing cellular immune responses to feline immunodefiency virus induced by DNA vaccination, Vaccine 18, 1118-1132 (2000).

554. J. Richardson, A. Moraillon, S. Baud, A. M. Cuisinier, P. Sonigo, and G. Pancino, Enhancement of feline immunodeficiency virus (FIV) infection after DNA vaccination with the FIV envelope, J. Virol. 71, 9640-9649 (1997).

555. J. Richardson, S. Broche, S. Baud, T. Leste-Lasserre, F. Femenia, D. Levy, A. Moraillon, G. Pancino, and P. Sonigo, Lymphoid activation: a confounding factor in AIDS vaccine development?, J. Gen. Virol. 83, 2515-2521 (2002).

556. A. M. Cuisinier, V. Mallet, A. Meyer, C. Caldora, and A. Aubert, DNA vaccination using expression vectors carrying FIV structural genes induces immune response against feline immunodeficiency virus, Vaccine 15, 1085-1094 (1997).

557. P. Gonin, A. Fournier, W. Oualikene, A. Moraillon, and M. Eloit, Immunization trial of cats with a replication-defective adenovirus type 5 expressing the ENV gene of feline immunodeficiency virus, Vet. Microbiol. 45, 393-401 (1995).

558. M. J. Burkhard, L. Valenski, S. Leavell, G. A. Dean, and W. A. Tompkins, Evaluation of FIV protein-expressing VEE-replicon vaccine vectors in cats, Vaccine 21, 258-268 (2002).

559. E. J. Tijhaar, W. Huisman, R. C. Huisman, K. H. Siebelink, J. A. Karlas, A. de Ronde, R. van Herwijnen, F. R. Mooi, and A. D. Osterhaus, Salmonella typhimurium aroA recombinants and immune-stimulating complexes as vaccine candidates for feline immunodeficiency virus, J. Gen. Virol. 78, 3265-3275 (1997).

560. R. Stevens, K. E. Howard, S. Nordone, M. J. Burkhard, and G. A. Dean, Oral immunization with recombinant listeria monocytogenes controls virus load after vaginal challenge with feline immunodeficiency virus, J. Virol. 78, 8210-8218 (2004).

561. A. Kumar, S. Mukherjee, J. Shen, S. Buch, Z. Li, I. Adany, Z. Liu, W. Zhuge, M. Piatak, J. D. Lifson, H. McClure, and O. Narayan, Immunization of macaques with live simian human immunodeficiency virus (SHIV) vaccines conferred protection against AIDS induced by homologous and heterologous SHIVs and simian immunodeficiency virus, Virology 301, 189-205 (2002).

562. A. Harmache, C. Vitu, F. Guiguen, P. Russon, G. Bertoni, M. Pepin, R. Vigne, and M. Susan, Priming with tat-deleted caprine arthritis encphalitis virus (CAEV) proviral DNA or live virus protects goats from challenge with pathogenic CAEV, J. Virol. 72, 6796-6804 (1998).

563. F. Li, J. K. Craigo, L. Howe, J. D. Steckbeck, S. Cook, C. J. Issel, and R. C. Montelaro, A live attenuated equine infectious anemia virus proviral vaccine with a modified $\mathrm{S} 2$ gene provides protection from detectable infection by intravenous virulent virus challenge of experimentally inoculated horses, J. Virol. 77, 7244-7253 (2003). 
564. M. Kohmoto, T. Miyazawa, E. Sato, K. Uetsuka, Y. Nishimura, Y. Ikeda, G. Inada, K. Doi, and T. Mikami, Cats are protected against feline immunodeficiency virus infection following vaccination with a homologous AP-1 binding site-deleted mutant, Arch. Virol. 143, 1839-1845 (1998).

565. S. VandeWoude, C. A. Hageman, S. J. O'Brien, and E. A. Hoover, Nonpathogenic lion and puma lentiviruses impart resistance to superinfection by virulent feline immunofeciency virus, J.A.I.D.S. 29, 1-10 (2002).

566. R. C. Desrosiers, Prospects for an AIDS vaccine, Nat. Med. 10, 221-223 (2004).

567. M. L. Raabe, C. J. Issel, S. J. Cook, R. F. Cook, B. Woodson, and R. C. Montelaro, Immunization with a recombinant envelope protein (rgp90) of EIAV produces a spectrum of vaccine efficacy ranging from lack of clinical disease to severe enhancement, Virology 245, 151-162 (1998).

568. R. Merat, H. Raoul, T. Leste-Lasserre, P. Sonigo, and G. Pancino, Variable constraints on the principal immunodominant domain of the transmembrane glycoprotein of human immunodeficiency virus type 1, J. Virol. 73, 5698-5706 (1999).

569. W. Huisman, E. J. Schrauwen, S. D. Pas, J. A. Karlas, G. F. Rimmelzwaan, and A. D. Osterhaus, Antibodies specific for hypervariable regions 3 to 5 of the feline immunodeficiency virus envelope glycoprotein are not solely responsible for vaccine-induced acceleration of challenge infection in cats, J. Gen. Virol. 85, 1833-1841 (2004).

570. S. I. Staprans, A. P. Barry, G. Silvestri, J. T. Safrit, N. Kozyr, B. Sumpter, H. Nguyen, H. McClure, D. Montefiori, J. I. Cohen, and M. B. Feinberg, Enhanced SIV replication and accelerated progression to AIDS in macaques primed to mount a CD4 T-cell response to the SIV envelope protein, Proc. Natl. Acad. Sci. USA 101, 13026-13031 (2004).

571. R. C. Cronn, K. M. Remington, B. D. Preston, and T. W. North, Inhibition of reverse transcriptase from feline immunodeficiency virus by analogs of $2^{\prime}$-deoxyadenosine- $5^{\prime}$ triphosphate, Biochem. Pharmacol. 44, 1375-1381 (1992).

572. H. Egberink, M. Borst, H. Niphuis, J. Balzarini, H. Neu, H. Schellekens, E. De Clercq, M. Horzinek, and M. Koolen, Suppression of feline immunodeficiency virus infection in vivo by 9-(2-phosphonomethoxyethyl)adenine, Proc. Natl. Acad. Sci. USA 87, 30873091 (1990).

573. K. Hartmann, J. Balzarini, J. Higgins, E. De Clercq, and N. C. Pedersen, In vitro activity of acyclic nucleoside phosphonate derivatives against feline immunodeficiency vius in Crandell feline kidney cells and feline peripheral blood lymphocytes, Antiviral Chem. Chemother. 6, 13-19 (1994).

574. T. W. North and R. A. LaCasse, Testing HIV-1 drugs in the FIV model, Nat. Med. 1, 410-411 (1995).

575. T. W. Vahlenkamp, A. De Ronde, J. Balzarini, L. Naesens, E. De Clercq, M. J. van Eijk, M. C. Horzinek, and H. F. Egberink, (R)-9-(2-phosphonylmethoxypropyl)-2,6diaminopurine is a potent inhibitor of feline immunodeficiency virus infection, Antimicrob. Agents Chemother. 39, 746-749 (1995).

576. L. M. Stolk and J. F. Luers, Increasing number of anti-HIV drugs but no definite cure. Review of anti-HIV drugs, Pharm. World Sci. 26, 133-136 (2004).

577. J. M. Gobert, K. M. Remington, Y. Q. Zhu, and T. W. North, Multiple-drug-resistant mutants of feline immunodeficiency virus selected with $2^{\prime}, 3^{\prime}$-dideoxyinosine alone and in combination with 3'-azido-3'-deoxythymidine, Antimicrob. Agents Chemother. 38, 861-864 (1994).

578. Y. Q. Zhu, K. M. Remington, and T. W. North, Mutants of feline immunodeficiency virus resistant to $2^{\prime}, 3^{\prime}$-dideoxy-2', 3'-didehydrothymidine, Antimicrob. Agents Chemother. 40, 1983-1987 (1996).

579. H. K. Medlin, Y. Q. Zhu, K. M. Remington, T. R. Phillips, and T. W. North, Selection and characterization of a mutant of feline immunodeficiency virus resistant to $2^{\prime}, 3^{\prime}$ dideoxycytidine, Antimicrob. Agents Chemother. 40, 953-957 (1996). 
580. N. R. Smyth, M. Bennett, R. M. Gaskell, C. McCracken, C. A. Hart, and J. L. Howe, Effect of 3'azido-2', 3'-deoxythymidine (AZT) on experimental feline immunodeficiency virus infection in domestic cats, Res. Vet. Sci. 57, 220-224 (1994).

581. K. Hartmann, A. Donath, B. E. Beer, H. F. Egberink, M. C. Horzinek, H. Lutz, G. Hoffmann-Fezer, I. Thum, and S. Thefeld, Use of two virustatica (AZT, PMEA) in the treatment of FIV and of FeLV seropositive cats with clinical symptoms, Vet. Immunol. Immunopathol. 35, 167-175 (1992).

582. K. Hartmann, A. Donath, and W. Kraft, AZT in the teatment of feline immunodeficiency virus infection, Part 1, Feline Pract. 23, 16-21 (1995).

583. K. Hartmann, A. Donath, and W. Kraft, AZT in the teatment of feline immunodeficiency virus infection, Part 2, Feline Pract. 23, 13-20 (1995).

584. K. A. Hayes, L. J. Lafrado, J. G. Erickson, J. M. Marr, and L. E. Mathes, Prophylactic ZDV therapy prevents early viremia and lymphocyte decline but not primary infection in feline immunodeficiency virus-inoculated cats, J. Acquir. Immune Defic. Syndr. 6, 127-134 (1993).

585. K. A. Hayes, J. G. Wilkinson, R. Frick, S. Francke, and L. E. Mathes, Early suppression of viremia by ZDV does not alter the spread of feline immunodeficiency virus infection in cats, J. Acquir. Immune Defic. Syndr. 9, 114-122 (1995).

586. J. Meers, G. M. del Fierro, R. B. Cope, H. S. Park, W. K. Greene, and W. F. Robinson, Feline immunodeficiency virus infection: plasma, but not peripheral blood mononuclear cell virus titer is influenced by zidovudine and cyclosporine, Arch. Virol. 132, 67-81 (1993).

587. K. A. Hayes, A. J. Phipps, S. Francke, and L. E. Mathes, Antiviral therapy reduces viral burden but does not prevent thymic involution in young cats infected with feline immunodeficiency virus, Antimicrob. Agents Chemother. 44, 2399-2405 (2000).

588. M. Arai, D. D. Earl, and J. K. Yamamoto, Is AZT/3TC therapy effective against FIV infection or immunopathogenesis?, Vet. Immunol. Immunopathol. 85, 189-204 (2002).

589. K. Hartmann, M. Kuffer, J. Balzarini, L. Naesens, M. Goldberg, V. Erfle, F. D. Goebel, E. De Clercq, J. Jindrich, A. Holy, N. Bischofberger, and W. Kraft, Efficacy of the acyclic nucleoside phosphonates (S)-9-(3-fluoro-2-phosphonylmethoxypropyl)adenine (FPMPA) and 9-(2-phosphonylmethoxyethyl)adenine (PMEA) against feline immunodeficiency virus, J. Acquir. Immune Defic. Syndr. Hum. Retrovirol. 17, 120-128 (1998).

590. M. S. Philpott, J. P. Ebner, and E. A. Hoover, Evaluation of 9-(2-phosphonylmethoxyethyl) adenine therapy for feline immunodeficiency virus using a quantitative polymerase chain reaction, Vet. Immunol. Immunopathol. 35, 155-166 (1992).

591. L. R. Bisset, H. Lutz, J. Boni, R. Hofmann-Lehmann, R. Luthy, and J. Schupbach, Combined effect of zidovudine (ZDV), lamivudine (3TC) and abacavir (ABC) antiretroviral therapy in suppressing in vitro FIV replication, Antiviral Res. 53, 35-45 (2002).

592. T. Lee, V. D. Le, D. Lim, Y. C. Lin, G. M. Morris, A. L. Wong, A. J. Olson, J. E. Elder, and C. H. Wong, Development of a new type of protease inhibitors, efficacious against FIV and HIV variants, J. Am. Chem. Soc. 121, 1145-1155 (1999).

593. M. Li, G. M. Morris, T. Lee, G. S. Laco, C. H. Wong, A. J. Olson, J. H. Elder, A. Wlodawer, and A. Gustchina, Structural studies of FIV and HIV-1 proteases complexed with an efficient inhibitor of FIV protease, Proteins 38, 29-40 (2000).

594. S. De Rozieres, C. H. Swan, D. A. Sheeter, K. J. Clingerman, Y. C. Lin, S. HuitronResendiz, S. J. Henriksen, B. E. Torbett, and J. H. Elder, Assessment of FIV-C infection of cats as a function of treatment with the protease inhibitor, TL-3, Retrovirology 1, 38 (2004).

595. R. J. Medinas, D. M. Lambert, and W. A. Tompkins, C-Terminal gp40 peptide analogs inhibit feline immunodeficiency virus: cell fusion and virus spread, J. Virol. 76, 9079-9086 (2002). 
596. C. T. Wild, D. C. Shugars, T. K. Greenwell, C. B. McDanal, and T. J. Matthews, Peptides corresponding to a predictive alpha-helical domain of human immunodeficiency virus type 1 gp41 are potent inhibitors of virus infection, Proc. Natl. Acad. Sci. USA 91, 9770-9774 (1994).

597. S. G. Peisajovich and Y. Shai, Viral fusion proteins: multiple regions contribute to membrane fusion, Biochem. Biophsy. Acta. 1614, 122-129 (2003).

598. A. Mergia, J. Blackwell, and S. Chari, Inhibition of FIV replication by a ribozyme that targets the Rev response element, AIDS Res. Hum. Retroviruses 13, 1333-1339 (1997).

599. K. De Mari, L. Maynard, A. Sanquer, B. Lebreux, and H. M. Eun, Therapeutic effects of recombinant feline interferon-omega on feline leukemia virus (FeLV)-infected and FeLV/feline immunodeficiency virus (FIV)-coinfected symptomatic cats, J. Vet. Intern. Med. 18, 477-482 (2004).

600. M. Arai, J. Darman, A. Lewis, and J. K. Yamamoto, The use of human hematopoietic growth factors (rhGM-CSF and rhEPO) as a supportive therapy for FIV-infected cats, Vet. Immunol. Immunopathol. 77, 71-92 (2000).

601. C. R. Gregory, J. E. Barlough, R. Clark, S. M. Griffey, J. D. Patz, P. Jardieu, and N. C. Pedersen, Effects of insulin-like growth factor-1 and AZT in cats experimentally infected with FIV, Feline Pract. 25, 23-31 (1997).

602. C. H. Pontzer, J. K. Yamamoto, F. W. Bazer, T. L. Ott, and H. M. Johnson, Potent antifeline immunodeficiency virus and anti-human immunodeficiency virus effect of IFNtau, J. Immunol. 158, 4351-4357 (1997).

603. C. M. Leutenegger, J. B. Huder, C. N. Mislin, F. Lahrtz, R. Hofmann-Lehmann, N. C. Pedersen, and H. Lutz, Molecular characterization of feline interleukin 16: chemotactic activity and effect on feline immunodeficiency virus infection and/or replication, AIDS Res. Hum. Retroviruses 16, 569-575 (2000).

604. S. L. Pett and A. D. Kelleher, Cytokine therapies in HIV-1 infection: present and future, Expert Rev. Anti Infect. Ther. 1, 83-96 (2003).

605. J. D. Siliciano and R. F. Siliciano, A long-term latent reservoir for HIV-1: discovery and clinical implications, J. Antimicrob. Chemother. 54, 6-9 (2004).

606. M. A. Curran and G. P. Nolan, Nonprimate lentiviral vectors, Curr. Top. Microbiol. Immunol. 261, 75-105 (2002).

607. D. T. Saenz and E. M. Poeschla, FIV: from lentivirus to lentivector, J. Gene Med. 6, S95-S104 (2004).

608. N. Loewen, M. Fautsch, and M. Peretz, Genetic modification of human trabecular meshwork with lentiviral vectors, Hum. Gene Ther. 12, 2109-2119 (2001).

609. J. J. Song, B. Lee, J. W. Chang, J. Kim, Y. K. Kwon, and H. Lee, Optimization of vesicular stomatitis virus-G pseudotyped feline immunodeficiency virus vector for minimized cytotoxicity with efficient gene transfer, Virus Res. 93, 25-30 (2003).

610. M. A. Curran, S. M. Kaiser, P. L. Achacoso, and G. P. Nolan, Efficient transduction of nondividing cells by optimized feline immunodeficiency virus vectors, Mol. Ther. 1, 31-38 (2000).

611. N. Loewen, D. Leske, and Y. Chen, Comparison of wild type and class I integrase mutantFIV vectors in retina demonstrates sustained expression of integrated transgenes in retinal pigment epithelium, J. Gene Med. 5, 1009-1017 (2003).

612. N. Loewen, R. Barraza, T. Whitwam, D. T. Saenz, I. Kemler, and E. M. Poeschla, FIV vectors, Methods Mol. Biol. 229, 251-271 (2003).

613. M. C. Barr, P. P. Calle, M. E. Roelke, and F. W. Scott, Feline immunodeficiency virus infection in nondomestic felids, J. Zoo. Wildl. Med. 20, 265-272 (1989).

614. M. C. Barr, L. Zou, F. Long, W. A. Hoose, and R. J. Avery, Proviral organization and sequence analysis of feline immunodeficiency virus isolated from a Pallas cat, Virology 228, 84-91 (1997). 
615. M. Carpenter, E. Brown, and M. Culver, Genetic and phylogenetic divergence of feline immunodeficiency virus in the puma (Puma concolor), J. Virol. 70, 6682-6693 (1996).

616. R. J. Langley, V. M. Hirsch, S. J. O’Brien, D. Adger-Johnson, R. M. Goeken, and R. Olmsted, Nucleotide sequence analysis of puma lentivirus (PLV-14): genomic organization and relationship to other lentiviruses, Virology 202, 853-864 (1994).

617. S. VandeWoude, S. J. O’Brien, K. Langelier, W. D. Hardy, J. P. Slattery, E. E. Zuckerman, and E. A. Hoover, Growth of lion and puma lentiviruses in domestic cat cells and comparisons with FIV, Virology 233, 185-192 (1997).

618. J. L. Troyer, J. Pecon-Slattery, M. E. Roelke, L. Black, C. Packer, and S. J. O’Brien, Patterns of feline immunodeficiency virus multiple infection and genome divergence in a free-ranging population of African lions, J. Virol. 78, 3777-3791 (2004).

619. M. A. Carpenter and S. J. O'Brien, Coadaptation and immunodeficiency virus: lessons from the Felidae, Curr. Opin. Genet. Dev. 5, 739-745 (1995).

620. C. Packer, S. Alitzer, M. Appel, E. Brown, J. S. Martenson, S. J. O’Brien, M. Roelke-Parker, R. Hofmann-Lehmann, and H. Lutz, Viruses of the Serengeti: patterns of infection and mortality in lions, J. Anim. Ecol. 68, 1161-1178 (1999).

621. S. Goldstein, I. Ourmanov, C. R. Brown, B. E. Beer, W. R. Elkins, R. Plishka, A. BucklerWhite, and V. M. Hirsch, Wide range of viral load in healthy African green monkeys naturally infected with simian immunodeficiency virus, J. Virol. 74, 11744-11753 (2000).

622. V. M. Hirsch, G. Dapolito, R. M. Goeken, and B. Campbell, Phylogeny and natural history of the primate lentiviruses, SIV and HIV, Curr. Opin. Genet. Dev. 5, 798-806 (1997).

623. R. Shankarappa, J. B. Margolick, S. J. Gange, A. G. Rodrigo, D. Upchurch, H. Farzadegan, P. Gupta, C. R. Rinaldo, G. H. Learn, X. He, X. L. Huang, and J. I. Mullins, Consistent viral evolutionary changes associated with the progression of human immunodeficiency virus type 1 infection, J. Virol. 73, 10489-10502 (1999).

624. E. Pelletier, W. Saurin, R. Cheynier, N. L. Letvin, and S. Wain-Hobson, The tempo and mode of SIV quasispecies development in vivo calls for massive viral replication and clearance, Virology 20, 644-652 (1995).

625. M. C. Muller-Trutwin, S. Corber, M. D. Tavares, V. M. Herve, E. Nerrienet, M. C. GeorgesCourbot, W. Saurin, P. Sonigo, and F. Barre-Sinoussi, The evolutionary rate of nonpathogenic simian immunodeficiency virus (SIVagm) is in agreement with a rapid and continuous replication in vivo, Virology 223, 89-102 (1996).

626. T. Leitner and J. Albert, The molecular clock of HIV-1 unveiled through analysis of a known transmission history, Proc. Natl. Acad. Sci. USA 96, 10752-10757 (1999).

627. S. VandeWoude, S. J. O'Brien, and E. A. Hoover, Infectivity of lion and puma lentiviruses for domestic cats, J. Gen. Virol. 78, 795-800 (1997).

628. S. VandeWoude, C. L. Hageman, and E. A. Hoover, Domestic cats infected with lion or puma lentivirus develop anti-feline immunodeficiency virus immune responses, J. Acquir. Immune Defic. Syndr. 34, 20-31 (2003). 